University of South Florida

DIGITAL COMMONS

Digital Commons @ University of

@ UNIVERSITY OF SOUTH FLORIDA

South Florida

January 2020

\title{
Perfectionism, Negative Life Events, and Cognitive Appraisal: A Contextual Model of Perfectionism's Maladaptive Nature
}

Ansley M. Bender

University of South Florida

Follow this and additional works at: https://digitalcommons.usf.edu/etd

Part of the Clinical Psychology Commons

\section{Scholar Commons Citation}

Bender, Ansley M., "Perfectionism, Negative Life Events, and Cognitive Appraisal: A Contextual Model of Perfectionism's Maladaptive Nature" (2020). USF Tampa Graduate Theses and Dissertations.

https://digitalcommons.usf.edu/etd/8008

This Thesis is brought to you for free and open access by the USF Graduate Theses and Dissertations at Digital Commons @ University of South Florida. It has been accepted for inclusion in USF Tampa Graduate Theses and Dissertations by an authorized administrator of Digital Commons @ University of South Florida. For more information, please contact digitalcommons@usf.edu. 
Perfectionism, Negative Life Events, and Cognitive Appraisal: A Contextual Model of Perfectionism's Maladaptive Nature

by

Ansley M. Bender

A thesis submitted in partial fulfillment of the requirements for the degree of Master of Arts Department of Psychology College of Arts and Sciences University of South Florida

Major Professor: Marc Karver, Ph.D. Jack Darkes, Ph.D. Jamie Goldenberg, Ph.D.

Date of Approval:

November 22, 2019

Keywords: socially prescribed perfectionism, demoralization, suicidality, standards Copyright (C) 2019, Ansley M. Bender 


\section{Table of Contents}

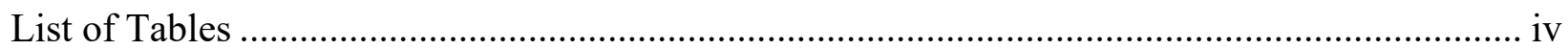

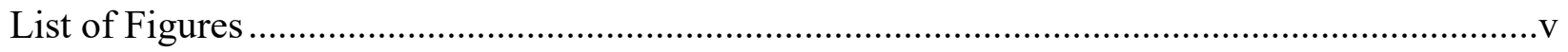

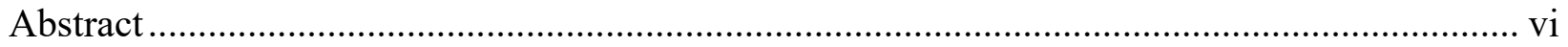

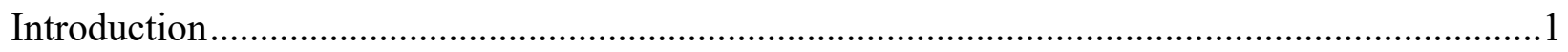

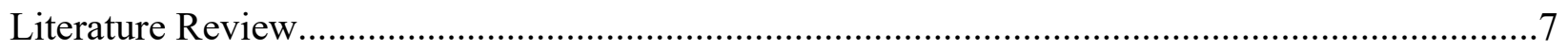

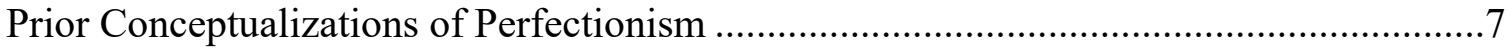

Unidimensional ...................................................................................... 7

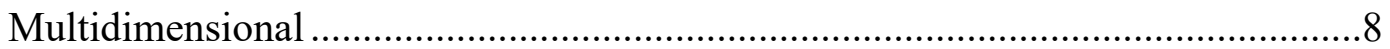

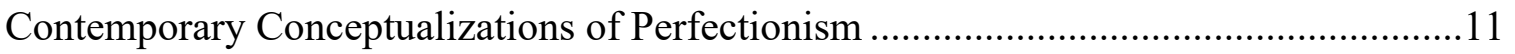

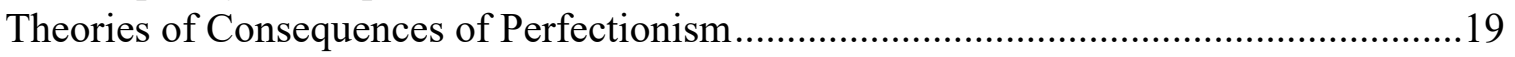

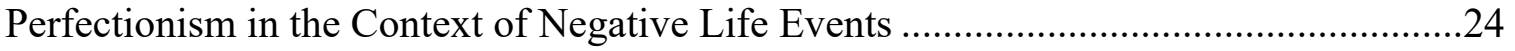

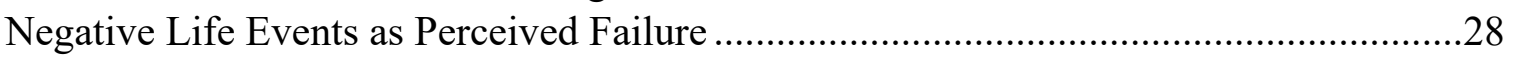

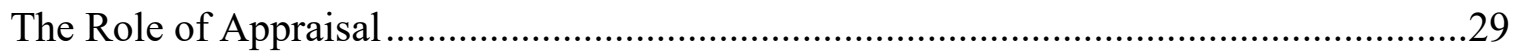

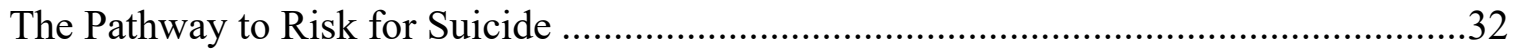

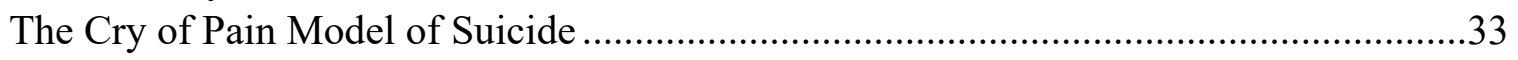

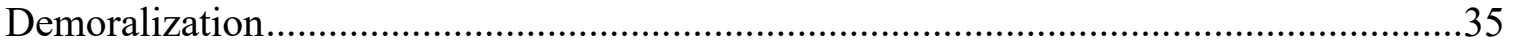

An Integrated Model of the Contextual Effects of Perfectionism ..................................40

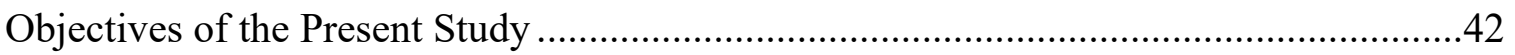

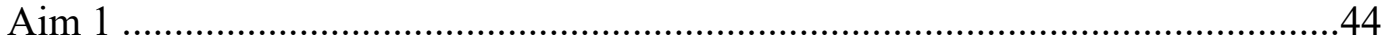

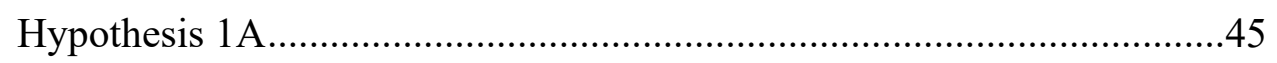

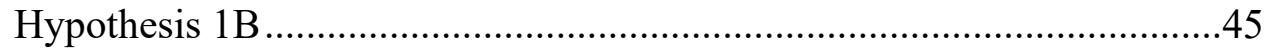

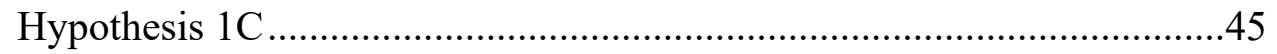

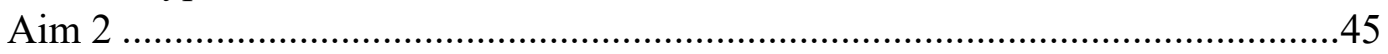

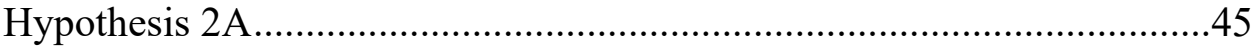

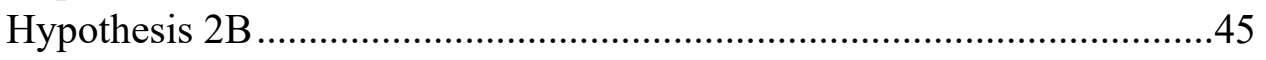

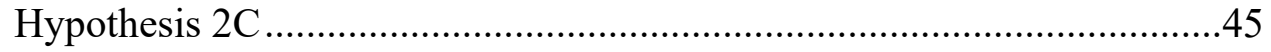

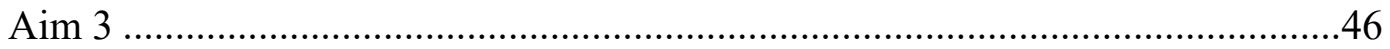

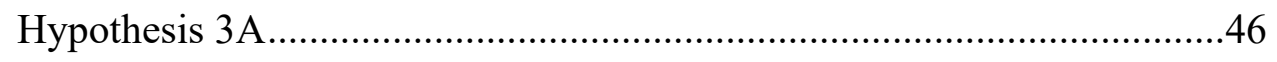

Hypothesis 3A (i) .............................................................46

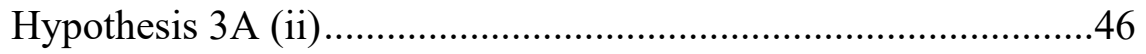

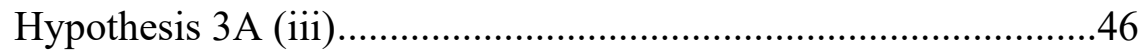

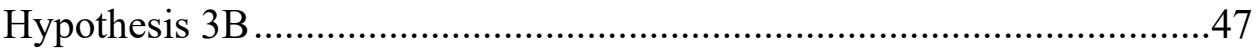

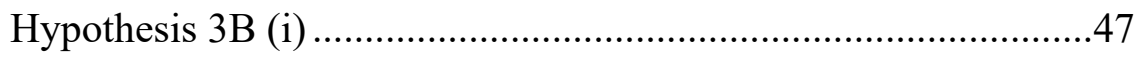




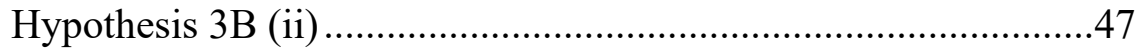

Hypothesis 3B (iii) ...............................................................47

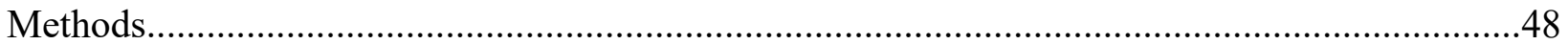

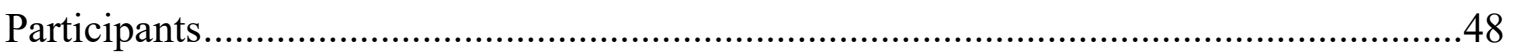

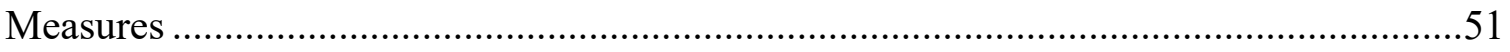

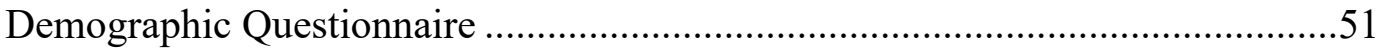

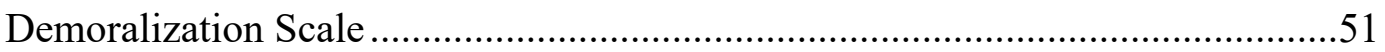

Multidimensional Perfectionism Scale .........................................................53

Life Events Scale for Students ........................................................................54

Scale for Assessing Appraisal Components ....................................................55

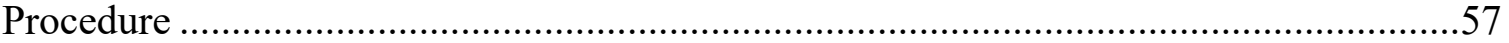

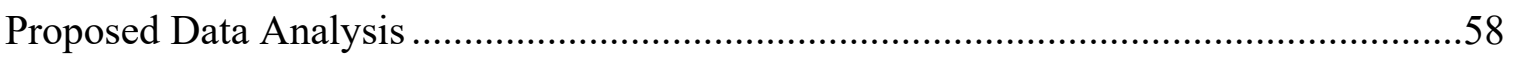

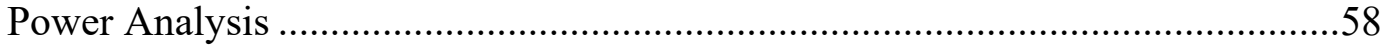

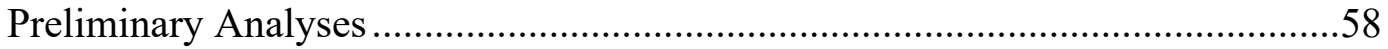

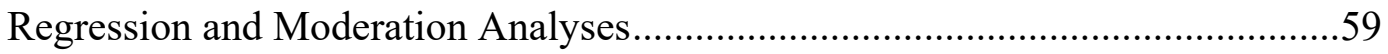

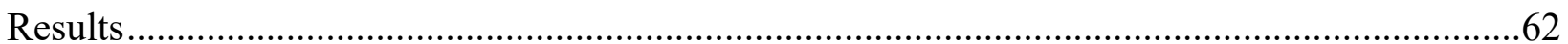

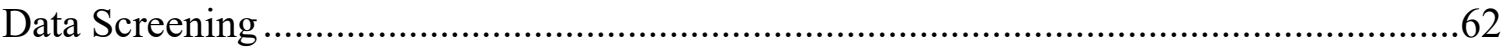

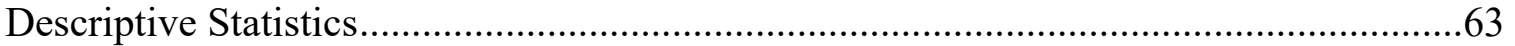

Multidimensional Perfectionism Scale .........................................................63

Life Events Scale for Students .....................................................................63

Scale for Assessing Appraisal Components ........................................................64

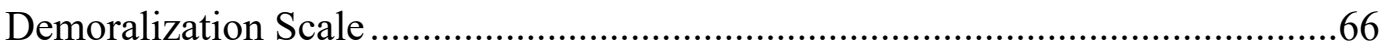

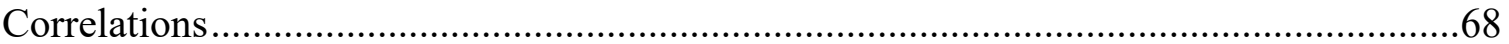

Relationship between Study Variables and Relevant Covariates ...................................68

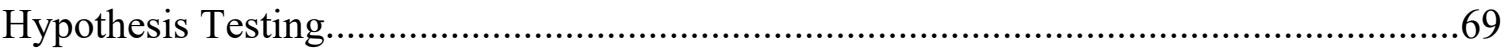

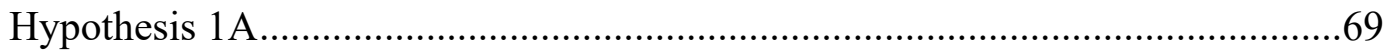

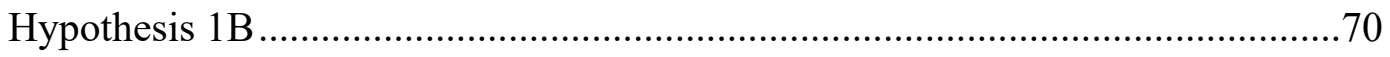

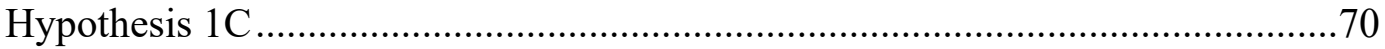

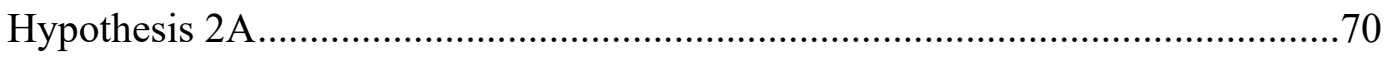

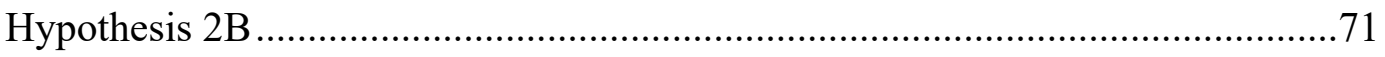

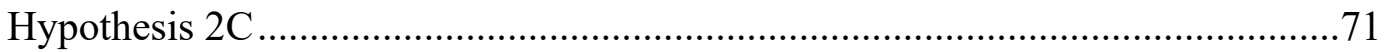

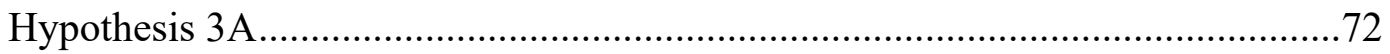

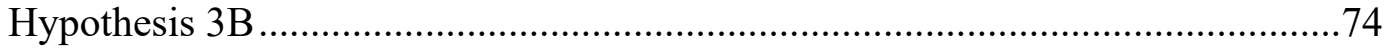

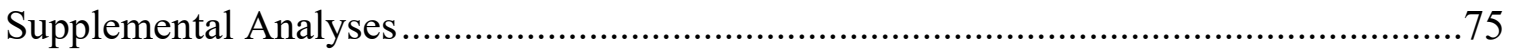

Main Effect of Socially Prescribed Perfectionism on Demoralization

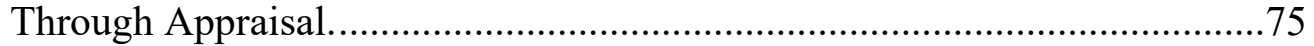

Main Effect of Self-Oriented Perfectionism on Demoralization Through

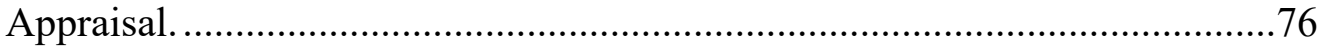

Examining the Effect of Socially Prescribed Perfectionism....................77

Main Effect of Self-Oriented Perfectionism on Demoralization Through Socially Prescribed Perfectionism......................................78 
Main Effect of Negative Life Events on Demoralization Through

Appraisal.

Mediated Effect of Socially Prescribed Perfectionism on Demoralization

Through Individual Appraisal Components. .................................................8

Mediated Effect of Self-Oriented Perfectionism on Demoralization

Through Individual Appraisal Components.

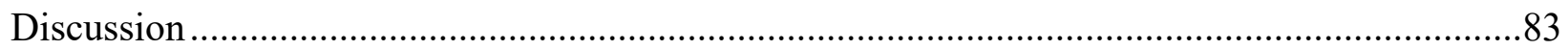

Socially Prescribed Perfectionism as a Robust Risk Factor .............................................83

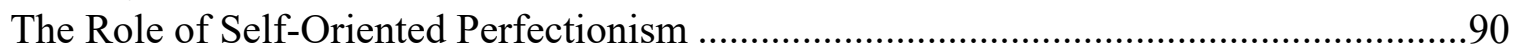

Differential Mediating Effects of Specific Appraisals.......................................................94

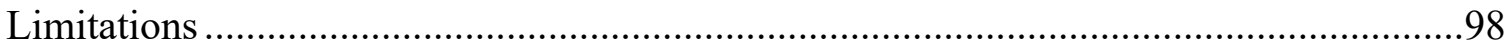

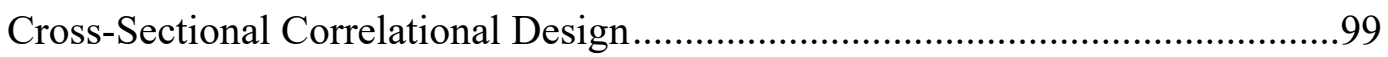

Use of Self-Report Survey Data …………………….....................................99

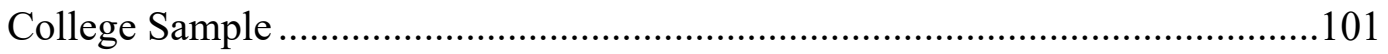

Summary and Future Directions ...................................................................................103

Socially Prescribed Perfectionism as a Robust Risk Factor ................................103

The Role of Self-Oriented Perfectionism ............................................................105

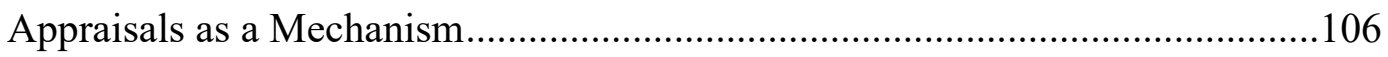

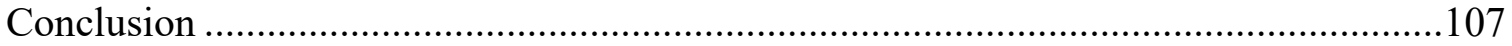

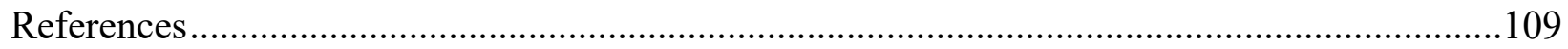

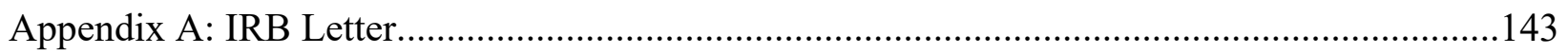




\section{List of Tables}

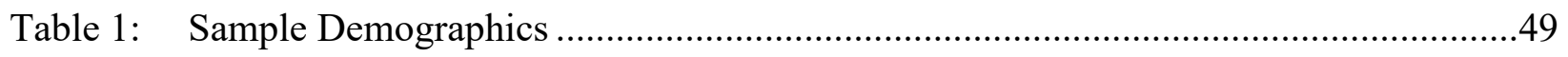

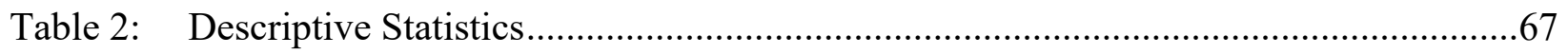

Table 3: Intercorrelations of Study Variables .............................................................68 


\section{List of Figures}

Figure 1: Hypothesized model of the relationship between perfectionism (predictor) and demoralization (dependent variable), in the context of negative life events (moderator) and through a pathway of appraisal as personal failures (mediator).

Figure 2: Statistical model of the mediated effect of perfectionism and negative life events on demoralization through appraisal.

Figure 3: Statistical model of the proposed effect of socially prescribed perfectionism and negative life events on demoralization through appraisal

Figure 4: Statistical model of the proposed effect of self-oriented perfectionism and negative life events on demoralization through appraisal

Figure 5: Statistical model of the proposed effect of self-oriented perfectionism on demoralization through appraisal

Figure 6: Statistical model of the proposed effect of self-oriented perfectionism on demoralization through socially prescribed perfectionism and appraisal

Figure 7: Statistical model of the proposed effect of socially prescribed perfectionism on demoralization through appraisal of falling short of standards

Figure 8: Statistical model of the proposed effect of self-oriented perfectionism on demoralization through appraisal of falling short of standards 


\begin{abstract}
Perfectionism is highly (Grzegorek, Slaney, Franze, \& Rice, 2004; Rice, Ashby, \& Gilman, 2011) and increasingly (Curran \& Hill, 2017) prevalent. Alarmingly, perfectionism is associated with various concerning correlates and outcomes, including suicide ideation and attempts (e.g. Smith, Sherry, et al., 2018). Whereas it is clear that perfectionism presents as a vulnerability for suicide, very little is known about when its maladaptive features may be particularly activated or by what pathway such a relationship exists. A preliminary, but underdeveloped, area of research suggests a role for negative life events in potentiating the effects of perfectionism, operating under the assumption that such events may approximate the aversive experience of failure (e.g. Dean, Range, \& Goggin, 1996). However, this assumption reveals an essential component — that of appraisal — which has been neglected in extant investigations of perfectionism's contextual effects. Given that appraisals may be more amenable to change than the occurrence of negative life events or the relatively stable trait of perfectionism, it is important to determine the extent to which appraisals may explain the effects of perfectionism, negative life events, and their interaction. The present study tested a model by which perfectionism would increasingly relate to demoralization in the context of negative life events through a pathway of appraising these events as personal failures. This model was partially supported; perfectionism predicted demoralization, but this relationship was not influenced by negative life events. Supplemental analyses indicated support for the mediating role of specific appraisal components. Clinical implications and deirections for future research are reviewed.
\end{abstract}




\section{Introduction}

Perfectionism, broadly defined as the combination of excessively high standards with an overly critical self-evaluation (Frost, Marten, Lahart, \& Rosenblate, 1990), is highly prevalent, with more than half of all college students classified as perfectionistic (Grzegorek et al., 2004). Research indicates that approximately one in four adolescents exhibit patterns of maladaptive perfectionism (Rice et al., 2011), but some findings place this estimate as high as $41 \%$ (Herman, Trotter, Reinke, \& Ialongo, 2011). Not only does perfectionism have a high base rate, but it appears to be rising steadily (Flett \& Hewitt, 2014). A recent meta-analysis suggests that perfectionism has increased by as much as $32 \%$ in the past 30 years; nearly two-thirds of current college students are above the mean level of perfectionism for college students in 1989 (Curran \& Hill, 2017). Longitudinal analyses corroborate these findings of increasing perfectionism (Portešová \& Urbánek, 2013). This may be attributable, in part, to cultural shifts, such as increasing meritocracy and competitive individualism, as well as changes in parenting practices that reinforce such perfectionism (Curran \& Hill, 2017). As perfectionism becomes increasingly prevalent, it is likewise increasingly important to understand its effect on mental health and overall wellbeing.

That perfectionism is increasing in prevalence is particularly concerning when contextualized by its negative correlates and outcomes. Perfectionism may ironically attenuate achievement in academic and professional settings, due to its relation to self-handicapping, lower achievement motivation, and difficulties with self-regulation (Hill, Hall, Duda, \& Appleton, 2011; Klibert, Langhinrichsen-Rohling, \& Saito, 2005; Mushquash \& Sherry, 2012). 
Perfectionism is similarly associated with physical health, including severity of headache pain, somatic complaints, and early death (Fry \& Debats, 2009; Kowal \& Pritchard, 1990; Wyatt \& Gilbert, 1998). Individuals who are perfectionistic experience greater negative affect and physiological reactivity after perceived or evaluated poor performance; these individuals, relative to controls, exhibit elevated dysphoria, anxiety, rumination, performance dissatisfaction, evaluated task importance, systolic blood pressure, and heart rate, and lowered self-esteem and positive affect, in such contexts (Besser, Flett, \& Hewitt, 2004; Besser, Flett, Hewitt, \& Guez, 2008). Regarding affective outcomes, higher perfectionism is associated with emotional distress, stress, pathological worry, shame, poorer self-confidence, body image avoidance, guilt, perceptions of failure, indecision, and deteriorated self-esteem (Bieling, Israeli, \& Antony, 2004; Dean et al., 1996; Handley, Egan, Kane, \& Rees, 2014; Hankin, Roberts, \& Gotlib, 1997; Hewitt, Flett, \& Ediger, 1995; Klibert et al., 2005). Perfectionism may further affect interpersonal functioning; evidence suggests perfectionism is associated with social dysfunction, loneliness, shyness, fear of negative evaluation, lower self-rated social skills, social stress, and ineffective expressions of anger (Flett, Hewitt, \& De Rosa, 1996; Hewitt et al., 2002; Hewitt, Flett, Sherry, \& Caelian, 2006; Pacht, 1984; Wyatt \& Gilbert, 1998). Perfectionism appears to disrupt effective coping, as it has been found to relate to deficits in problem solving and poorer cognitive, emotional, and behavioral coping (Flett, Hewitt, Blankstein, Solnik, \& Van Brunschot, 1996). Perfectionism is therefore associated with a variety of pernicious outcomes; its effects may further extend into the realm of psychopathology.

Beyond these broad negative correlates, perfectionism also confers risk for specific clinical symptoms and features. Elevated perfectionism is associated with body dysmorphic disorder (Buhlmann, Etcoff, \& Wilhelm, 2008) and eating disorders and disordered eating 
(Bardone-Cone, 2007; Brown, Parman, Rudat, \& Craighead, 2012; Downey \& Chang, 2007; Gross, Rosen, Leitenberg, \& Willmuth, 1986; Sassaroli et al., 2008). It likewise may relate to alcohol and drug abuse (Hewitt \& Flett, 1991b), symptoms of posttraumatic stress disorder (Egan, Hattaway, \& Kane, 2014), and risk for relapse in persons with schizophrenia (Vaughn \& Leff, 1981). Regarding personality problems, perfectionism has been linked to compulsive, paranoid, dependent, avoidant, schizotypal, antisocial, and passive aggressive personality features (Hewitt, Flett, \& Turnbull, 1992). Perfectionism is further associated with anxiety and related disorders, including generalized anxiety (Bieling et al., 2004; Handley et al., 2014; Hewitt \& Flett, 1991b; Juster et al., 1996; O'Connor, Rasmussen, \& Hawton, 2010), social anxiety (Antony, Purdon, Huta, \& Swinson, 1998; Juster et al., 1996; Saboonchi, Lundh, \& Öst, 1999), anxiety sensitivity (Flett, Greene, \& Hewitt, 2004), agoraphobia (Iketani et al., 2002), obsessive-compulsive disorder (Antony et al., 1998; Buhlmann et al., 2008; Frost \& Steketee, 1997; Hewitt \& Flett, 1991b; Sassaroli et al., 2008), and panic disorder (Antony et al., 1998). Perfectionism exhibits a robust relationship with depression (Bieling et al., 2004; Enns \& Cox, 2005; Flett, Hewitt, Blankstein, \& Mosher, 1995; Frost, Heimberg, Holt, Mattia, \& Neubauer, 1993; Hewitt \& Flett, 1993; Hewitt \& Dyck, 1986; O'Connor et al., 2010; Rasmussen, O’Connor, \& Brodie, 2008; Sassaroli et al., 2008; Wyatt \& Gilbert, 1998) and may predict persistence of depressive symptoms longitudinally (Enns \& Cox, 2005; Hooley \& Teasdale, 1989). These negative effects of perfectionism, both broad and clinical, may deplete an individual's resilience over time, leading to more severe consequences.

Perhaps most alarmingly, a growing body of research implicates perfectionism in risk for suicidal ideation and behaviors (Hamilton \& Schweitzer, 2000; Hewitt, Flett, \& Weber, 1994; O'Connor et al., 2010; Rasmussen et al., 2008). Perfectionism is related to nonsuicidal self-injury 
(NSSI; Brocklesby, 2017; Claes, Soenens, Vansteenkiste, \& Vandereycken, 2012; Hoff \& Muehlenkamp, 2009), particularly frequency of NSSI (Miskey, Hill, \& Huelsman, 2012). Qualitatively, case conceptualizations of suicide attempters using Plan analysis (Caspar, 2007; Grawe, 1980) have revealed themes of high personal standards, high perceived expectations from others, and fear of failure (Brüdern et al., 2015); similarly, results of psychological autopsies have suggested that $71.4 \%$ of those who died by suicide had expressed perceptions of high demands and expectations (Törnblom, Werbart, \& Rydelius, 2013). Empirically, perfectionism is associated with suicidal intent (Hewitt, Flett, \& Turnbull-Donovan, 1992) and, particularly in the context of life stress, may account for variance in suicidal ideation and suicide potential above and beyond depression, hopelessness, and prior attempts (Dean et al., 1996; Hewitt, Caelian, Chen, \& Flett, 2014; Hewitt et al., 1994). Perfectionism may discriminate between persons who have engaged in self-injurious behavior and hospital controls, above and beyond hopelessness, depression, and anxiety (Hunter \& O'Connor, 2003). Perfectionism is likewise associated with suicidal motivation, suicide preparation, and occurrence, number of lifetime, and severity of suicide attempts (Flamenbaum \& Holden, 2007; Hewitt, Norton, Flett, Callander, \& Cowan, 1998; Smith, Sherry, et al., 2018). However, much of this research has relied on cross-sectional methodologies which limits causal inferences.

Importantly, there is evidence to suggest that the relationship between perfectionism and suicide related variables may be more than just correlational. It has been suggested that the causal pathway may originate with perfectionism. Indeed, perfectionism predicts prospective increases in variables implicated in risk for suicide, such as hopelessness and psychological symptoms, including depression, anxiety, and hostility (Chang \& Rand, 2000; Enns, Cox, Sareen, \& Freeman, 2001; Flett et al., 1995; Smith et al., 2016). Additionally, longitudinal 
research suggests perfectionism may interfere with treatment progress (Blatt, Zuroff, Quinlan, \& Pilkonis, 1996; Jacobs et al., 2009), with implications for persistence of suicidal ideation over time (Jacobs et al., 2009). Perfectionism further predicts NSSI behavior prospectively (Brocklesby, 2017). Concerning variables more proximally related to suicide risk, perfectionism predicts longitudinal suicidal ideation, controlling for baseline ideation (Beevers \& Miller, 2004; Jacobs et al., 2009; Smith, Sherry, et al., 2018). Perfectionism, therefore, is associated with numerous suicide related variables, and research supports a directional pathway from perfectionism to these outcomes.

Thus, perfectionism is prevalent in a large portion of the population, with this prevalence steadily increasing over time. Perfectionism appears to be an established risk factor for myriad negative correlates and outcomes, with a growing body of research linking perfectionism to risk for suicide prospectively. Far less is known, however, about the contexts under which perfectionism is particularly maladaptive. Indeed, perfectionism may at times be a desirable trait, insomuch as it is associated with organization, goal-setting, and motivation (Amodeo, 2014; Hewitt \& Flett, 1991b). Given perfectionism's association with severe psychopathology and risk for suicidal thoughts and behaviors, yet, also with adaptive processes, it is imperative to better understand what factors may activate, potentiate, or even negate its possibly maladaptive effects. In particular, there is a dearth of research investigating the role of appraisal style in these processes. To the extent that appraisal may mediate perfectionism's influence, it is worth considering as an additional point of intervention. Moreover, the mechanism by which perfectionism confers risk for suicide specifically remains unclear; there is a paucity of theoretically informed mediators proposed or tested in the literature to adequately explain this 
relationship. The current study therefore seeks to investigate not only when perfectionism is maladaptive, but also why it may pose as a risk for suicide. 


\section{Literature Review}

\section{Prior Conceptualizations of Perfectionism}

Unidimensional. Initial conceptualizations of perfectionism often defined the construct as a unidimensional personality trait. Hollender (1965) broadly considered perfectionism to be characterized by self-imposed expectations for performance that exceeded the demands of the situation. This understanding of perfectionism is intrapersonal in that the individual is responsible for both producing the standards and for evaluating progress towards meeting these standards. Burns (1980) likewise considered perfectionism a unidimensional construct involving compulsive striving towards often unobtainable goals and standards; similar to Hollender, Burns proposed an intrapersonal definition of perfectionism in which individuals set high standards for themselves. Importantly, Burns distinguished ordinary adaptive striving from perfectionism; whereas he considered the former to be characterized by satisfaction with goal pursuit and acceptance of failures and setbacks, he proposed the latter would be characterized by a relentless pursuit of high standards, to the point of engaging in self-defeating behavior, and

disproportionate negative reactions to perceived failure and inadequacy (Burns, 1980).

The definitions proposed by Hollender and Burns are informative, to the extent that they capture the intrapersonal features of perfectionism. Both researchers invoked the role of unrealistic demands, expectations, and standards that the individual places upon the self; the idea that unrealistic standards are inherent in perfectionism continues today. Their conceptualizations of perfectionism further began to expose the destructive effects of a construct that may otherwise appear to be adaptive. That perfectionism confers risk for various negative outcomes continues to 
be supported, empirically and theoretically (e.g. Brocklesby, 2017; Frost et al., 1990; Hewitt \& Flett, 1991b; Smith, Sherry, et al., 2018; Smith et al., 2016). However, despite these contributions, the definitions of perfectionism proposed by these early researchers fall short of adequately representing the complexity of the construct. Indeed, their proposed etiologies and Burns's distinguishing of adaptive from maladaptive striving suggest a multifaceted construct. That is, both Hollender and Burns invoked the role of family systems, and other social reinforcement, in the development and maintenance of perfectionism. Hollender proposed a model whereby children with insecure attachment styles engage in perfectionistic practices to obtain approval of inconsistent or overly critical parents (Hollender, 1965); Burns similarly suggested the role of interpersonal dynamics in reinforcing perfectionistic striving, and further described the role of maladaptive cognitive processes, such as dichotomous thinking, in maintaining this striving (Burns, 1980). It is conceivable, then, that perfectionism may have both intra- and interpersonal dimensions. Moreover, Burns delineates ordinary striving from the compulsive pursuit of unrealistic standards that characterizes perfectionism. Whereas Burns considers the former to be entirely distinct from perfectionism, it is possible that it is instead an adaptive presentation of perfectionism; perfectionism may be comprised of both positive and negative qualities and manifestations. Early unidimensional definitions of perfectionism therefore provided a useful starting point to begin conceptualizing the construct, including its etiology and maintenance, but failed to account for the complexity of this trait. The proposed role of interpersonal dynamics in the development and maintenance of perfectionism would suggest that standards, real or perceived, may stem from an individual or individuals other than the self; the distinguishing between adaptive versus compulsive striving would suggest that 
perfectionism may manifest in both positive and negative variants. Accordingly, theories that considered the complex nature of perfectionism began to receive more attention.

Multidimensional. Alongside, and even prior to, these unidimensional definitions of perfectionism, multidimensional conceptualizations of the construct were proposed. As understandings of perfectionism progressed, these multidimensional conceptualizations became more predominant, with researchers tending to dichotomize it into "neurotic" and "normal," or "maladaptive" and "adaptive," variants. As far back as 1931, Adler proposed that individuals who are perfectionistic may be parsed into normal and neurotic perfectionists (1931). Adler largely viewed perfectionism as a universal construct, in that he believed that humans constantly strive to improve and adapt. However, he, like Hollender and Burns, theorized that neurotic perfectionism was characterized by excessive self-discrepancy and disproportionate negative affect in response to failure. Further, long before Burns suggested an adaptive counterpart to perfectionism's compulsive striving, Adler offered the comparable proposal that normal perfectionists view failure more adaptively and react in constructive ways, such that they may continue pursuit of goals (1931). The utility of Adler's early conceptualization of perfectionism is reflected in its similarity to later multidimensional definitions.

Years later, Hamachek paralleled Adler's distinction of normal and neurotic perfectionists; in Hamachek's model, neurotic perfectionists were defined by their setting of unrealistic standards and engagement in rigid striving towards goals (1978). He further postulated that neurotic perfectionists are less satisfied with progress that they do make. In contrast, he proposed that normal perfectionists could choose to flexibly employ their selfimposed standards and that these perfectionists obtained satisfaction from pursuing their goals. Like Hollender, Burns, and Adler, Hamachek defined perfectionism as an intrapersonal construct 
in which the individual has developed high standards for the self. Like Adler, Hamachek proposed both normal and neurotic manifestations of perfectionism. Finally, like Hollender and Burns, Hamachek proposed that maladaptive perfectionism may develop in contexts of absent, conditional, or inconsistent support from parents. He additionally theorized that normal perfectionism developed through a different mechanism- that of modeling from parents (Hamachek, 1978). Hamachek added to a growing understanding of perfectionism as multidimensional; this conceptualization was further developed by Slaney.

More recently, Slaney et al. proposed a multidimensional conceptualization of perfectionism in which the trait may be distinguished into adaptive and maladaptive manifestations; whether perfectionism is positive or negative largely depends on how well individuals perceive themselves to be meeting their own standards for success (Slaney, Ashby, \& Trippi, 1995; Slaney, Rice, Mobley, Trippi, \& Ashby, 2001). Within this conceptualization, the adaptive qualities of perfectionism include high standards and a high degree of organization; perfectionism is believed to be maladaptive to the extent that the individual perceives and is concerned over self-discrepancy (Slaney et al., 1995). Although Slaney distinguished perfectionism into adaptive and maladaptive variants, these closely map onto the normal and neurotic presentations described by Adler and Hollender. Collectively, these researchers viewed perfectionism to be concerning when it is accompanied by rigid striving towards unrealistically high standards, excessive discrepancy between ideal and current self, and reactions to failure that are disproportionately negative; they believed perfectionism may be advantageous when the individual flexibly pursues high, but achievable, standards and copes with failure in a healthy manner. 
Together, Adler, Hamachek, and Slaney, among others, provided the groundwork for a more nuanced understanding of perfectionism. Specifically, these researchers proposed models of perfectionism comprised of both positive and negative qualities. These theories are an advancement over unidimensional models of perfectionism, and this improvement is reflected in their longevity; current conceptualizations of perfectionism continue to view the trait as multifaceted. However, these theories, like those of Hollender and Burns, viewed perfectionism as intrapersonal, even as they invoked interpersonal etiologies. Somewhat more recently, conceptualizations of perfectionism have been proposed that incorporate both intra- and interpersonal dimensions of perfectionism.

\section{Contemporary Conceptualizations of Perfectionism}

Frost and colleagues were perhaps the first to present a conceptualization of perfectionism that was both multidimensional and interpersonal. Specifically, they proposed that perfectionism was comprised of six dimensions, including personal standards, organization, concern over mistakes, doubting of actions, parental expectations, and parental criticism (Frost et al., 1990). Personal standards are comparable to achievement striving; on their own, these standards are not thought to be maladaptive. Likewise, organization, defined as orderliness and precision, is not considered maladaptive, but instead refers to how individuals approach achievement striving. In contrast, concern over mistakes refers to excessive concern about perceived inadequacies, to the point that striving is driven by fear of failure, rather than desire for achievement. Similarly, doubting of actions relates to a broad, persisting dissatisfaction with the performance of one's work, rather than doubt about a specific task or behavior; at elevated levels, this doubt may actually disrupt or delay engagement in striving, for fear of poor performance. Frost et al.'s interpersonal dimensions, parental expectations and parental criticism, 
refer to the belief that one's parents have set high standards for performance and the belief that falling short of these standards is unacceptable to parents. Collectively, these dimensions yield a more complex understanding of perfectionism; nonetheless, this model bears notable similarities to the theories that preceded it.

These six dimensions, though novel, are consistent with many of the previously proposed conceptualizations of perfectionism. In keeping with prior intrapersonal understandings of the construct (e.g. Adler, 1931; Burns, 1980; Hamachek, 1978; Hollender, 1965), Frost et al. defined perfectionism as the combination of high standards with an overly critical self-evaluation (1990). And, in keeping with prior multidimensional understandings of the construct (e.g. Adler, 1931; Hamachek, 1978), Frost et al. theorized that these critical self-evaluations were likely more closely related to maladaptive outcomes than the setting of high standards; perfectionistic tendencies, such as striving, may be adaptive, so long as they are not accompanied by an overly critical view of the self (Frost et al., 1990). Moreover, Frost's unique dimensions of perfectionism draw directly from prior models. Concern over mistakes, for instance, is found in Burns's (1980) proposed excessive negative reactions to perceived failure, as well as in the maladaptive cognitive processes that he theorized maintained perfectionism. It's likewise consistent with Hamachek's discrimination between normal and neurotic perfectionists (1978). Doubting of actions is found in Burns's (1980) and Adler's (1931) theories, in which perfectionism may lead to self-defeating behavior and disrupt effective goal pursuit. Similarly, Frost's personal standards and organization dimensions likely capture the normal and adaptive perfectionism proposed in prior (Adler, 1931; Hamachek, 1978) and subsequent (Slaney et al., 2001) theories. Even Frost's more novel dimensions, parental expectations and parental criticism, directly relate to the development of perfectionism as theorized by Hollender (1965), 
Burns (1980), Hamachek (1978), and others. Frost et al.'s model is thus grounded in the work of prior theorists. However, it improves upon these understandings in an important way.

Frost and colleagues expanded prior theories by including the role of parents in the definition of perfectionism, rather than simply in its etiology. Whereas prior researchers proposed that inconsistent or overly critical parenting styles lead to the development of perfectionism, perfectionism itself was considered to be contained within the individual and not characterized by parental demands or evaluations. Frost et al. were among the first to argue that interpersonal features may be integral components of maladaptive perfectionism in their own right, rather than simply antecedents to it. In other words, individuals who Adler and Hamachek would classify as having neurotic perfectionism would likely exhibit concern over mistakes and doubting of actions; however, their perfectionism may also involve an excessive preoccupation with meeting real or imagined standards from parents and with avoiding parental criticism. This drive to satisfy perceived parental pressures is a part of their perfectionism, rather than just a cause of it. Individuals who would be classified as having normal perfectionism, in contrast, may simply endorse elevated personal standards and organization, those intrapersonal aspects of perfectionism also described by Slaney. They may endorse much lower levels of the interpersonal dimensions, either because they perceive fewer demands and criticisms, or because they are less concerned with these pressures; they would likewise endorse lower levels of concern over mistakes and doubting of actions. The proposal that parental expectations and criticisms are a component of perfectionism, rather than separate from and anterior to it, is supported by research on the longevity of such influences; namely, the extent to which individuals endorse concern about excessive criticism and unrealistic standards from parents does not appear to decline with age, even when examined in individuals as old as 66 years old 
(Amodeo, 2014). To this end, Frost's model may better capture the enduring influence of interpersonal factors. Thus, the definition of perfectionism proposed by Frost and colleagues began to extend perfectionism into the realm of interpersonal dimensions. Despite this improvement, however, it maintained several limitations.

Frost's model is primarily limited by its emphasis on just parental relationships. While unarguably important to consider, parents are only one source of many from which perceived expectations and criticisms may stem. Frost's model neglects to account for how individuals may intuit or be prescribed expectations from friends, peers, coworkers, authority figures, siblings, or others. The conceptualization unnecessarily limits the focus to include only parents, and thus sacrifices valuable information on the roles that additional persons may have in one's perceptions of standards. A secondary limitation of this model is that the interpersonal facets of perfectionism are not theorized to affect the other maladaptive dimensions, but instead are theorized to operate independently alongside them. That is, the interpersonal features of perfectionism may not only comprise perfectionism but may also interact with other features of perfectionism to potentiate their effects; parental criticism and expectations may affect the presence or degree of one's concern over mistakes and self-doubt, a possibility that Frost et al. do not consider. Their model largely neglects to consider how source of standards, either self or others, may contribute to the adaptive or maladaptive nature of perfectionism. Within their conceptualization, an individual would conceivably be just as likely to exhibit excessive concern over mistakes and doubting of actions with or without interpersonal features. In reality, source of standards may not be entirely independent from the degree to which perfectionism is adaptive or maladaptive. Standards derived from others may be particularly likely to be accompanied by 
other deleterious features and outcomes of perfectionism. A theory that has developed alongside that of Frost et al. began to consider this possibility.

As Frost and colleagues developed a multidimensional, interpersonal definition of perfectionism, so too did Hewitt and Flett $(1989,1991 b)$. Whereas Frost's model defined perfectionism according to six unique dimensions, Hewitt and Flett proposed a model of perfectionism defined by three dimensions; namely, they characterized perfectionism by the extent to which individuals set high standards for themselves, the extent to which individuals perceive others have set high standards for them, and the extent to which individuals have set high standards for others. Hewitt and Flett labeled these, respectively, as self-oriented, socially prescribed, and other-oriented perfectionism. Although this categorization is perhaps the most unique among the various theories proposed, it bears some similarity to prior conceptualizations.

Hewitt and Flett's model incorporates features of perfectionism found in the models that preceded it. In particular, their model is comparable to that of Burns (1980); both models proposed that perfectionism was defined by setting and striving for unrealistic standards, and was accompanied by cognitive biases, including selective attention to failure, overgeneralization of failure, a skewed self-schema, all-or-none thinking, and overly critical self-evaluations (Hewitt \& Flett, 1991b). Moreover, Hewitt and Flett's self-oriented dimension of perfectionism closely resembles the unidimensional, intrapersonal definition of perfectionism proposed in prior theories (e.g. Burns, 1980; Hollender, 1965) in that individuals set, impose, and evaluate progress towards their own high standards. Finally, like Frost and colleagues (1990), Hewitt and Flett acknowledged the previously neglected interpersonal dimensions of perfectionism, incorporating perceived standards that stem from someone other than the self. Despite these 
commonalities, the model proposed by Hewitt and Flett deviates substantially from extant multidimensional definitions of perfectionism.

Whereas prior theories delineated adaptive from maladaptive perfectionism (Adler, 1931; Frost et al., 1990; Hamachek, 1978), Hewitt and Flett did not explicitly do so. Instead, they primarily divided perfectionism into self- and other-derived dimensions, and perfectionism's adaptive or maladaptive nature was therefore secondary to this classification. Specifically, they theorized that the extent to which perfectionism is adaptive or maladaptive is inherently associated, at least in part, with the source from which one derives standards; relative to past conceptualizations, self-oriented perfectionism may mostly closely mirror adaptive, normal perfectionism, whereas socially prescribed perfectionism may better resemble maladaptive, neurotic perfectionism (Amodeo, 2014). Although Hewitt and Flett improved upon prior interpersonal theories of perfectionism (Frost et al., 1990) by conferring greater influence to source of standards, this may also be considered a limitation of the model. Where Frost et al. neglected to consider how the source of standards influences perfectionism's nature, Hewitt and Flett arguably put too great an emphasis on the source of standards as the framework driving perfectionism's maladaptive qualities. That is, it may be too simplistic to ascribe the entirety of perfectionism's concerning effects to one's perceiving standards from external sources; it is almost certain that other factors play a role as well. And, though arguably more adaptive, setting standards for oneself is unlikely to be completely protective against perfectionism's negative consequences. However, it is important to note that they do not consider self-oriented perfectionism to be entirely devoid of negative correlates (Hewitt \& Flett, 1991b). Instead, they theorized that self-oriented perfectionism would be associated with these negative correlates to a lesser degree or with less frequency than is socially prescribed perfectionism. Further, the model 
does not preclude other factors from influencing perfectionism's maladaptive nature; this model would potentially allow for other variables such as negative life events, excessive self-criticism, or deficient coping to potentiate or moderate the quality of both self-oriented and socially prescribed perfectionism. And, perhaps most importantly, the data appear to support this proposed framework.

Research findings lend support to the model proposed by Hewitt and Flett, in that the source of one's standards may be largely influential in determining whether or not perfectionism is maladaptive, and to what extent. Whereas self-imposed standards are sometimes marked by negative correlates and outcomes, these findings are more equivocal, less significant, and of smaller magnitude, than findings related to perceived standards from others (e.g. Blankstein, Lumley, \& Crawford, 2007; Dean \& Range, 1999; Dean et al., 1996; Flett et al., 1995; Smith, Sherry, et al., 2018; Wyatt \& Gilbert, 1998). Socially prescribed perfectionism has been found to relate to negative affect, lowered self-esteem, lowered self-control, lowered achievement motivation, dysthymia, shame, and guilt, despite no significant associations found for selforiented perfectionism (Frost et al., 1993; Hewitt \& Flett, 1991b; Klibert et al., 2005). In contrast, self-oriented perfectionism, but not socially prescribed perfectionism, relates to greater positive affect (Frost et al., 1993). Across studies, socially prescribed perfectionism maintains a larger, and more significant, effect with psychopathology such as depression, anxiety disorders, obsessive-compulsive disorder, anorexia nervosa, and borderline personality disorder (Enns \& Cox, 1999; Flett et al., 2004; Flett, Hewitt, Blankstein, \& O'Brien, 1991; Hewitt \& Flett, 1991a, 1991b; Limburg, Watson, Hagger, \& Egan, 2017; Martin, Flett, Hewitt, Krames, \& Szanto, 1996; Shafran \& Mansell, 2001; Wyatt \& Gilbert, 1998). Alarmingly, socially prescribed perfectionism predicts prospective increases in suicidal ideation and presence and number of 
suicide attempts; self-oriented perfectionism appears to relate only to concurrent suicidal ideation, and this association is no longer significant after controlling for overlap with socially prescribed perfectionism (Smith, Sherry, et al., 2018). Thus, distinguishing socially prescribed from self-oriented perfectionism along the continuum of harm bears merit, given their respective relationships with negative consequences. This is further supported by comparisons of Frost's and Hewitt's conceptualizations.

When the theories of Frost and Hewitt are compared together, factor analysis supports a two-factor solution (Bieling et al., 2004; Frost et al., 1993). Hewitt's socially prescribed perfectionism groups with Frost's concern over mistakes, doubting of actions, parental criticism, and parental expectations, the four dimensions that Frost considers to be maladaptive. In contrast, Hewitt's self-oriented perfectionism groups with Frost's personal standards and organization, which both Frost (1990) and Slaney (2001) consider to be adaptive. Thus, although Frost's four maladaptive dimensions of perfectionism were theorized to operate parallel to and independent of one another, it instead appears that they are interrelated. The results of these factor analyses suggest that interpersonal features of perfectionism, whether Frost's parental criticisms and expectations or Hewitt's socially prescribed perfectionism, reflect the same underlying construct as, and are thus closely related to, other maladaptive features. Simultaneously, these interpersonal features do not appear to be closely related to the adaptive dimensions of perfectionism; the latter may reflect a distinct construct, of which self-oriented perfectionism is also a part. Therefore, the data suggest that the source of perceived standards is strongly related to both the presence and severity of perfectionism's maladaptive features and negative outcomes, with external sources posing as particularly deleterious. Although one may experience maladaptive perfectionism without interpersonal features, this may depend on the 
presence of other factors, such as excessive life stress (Flett et al., 1995; Hewitt, Flett, \& Ediger, 1996; Hewitt, Flett, \& Weber, 1994) or particularly dysfunctional cognitions (Besser, Flett, \& Hewitt, 2004; Chang \& Sanna, 2001; Kobori \& Tanno, 2005; Olson \& Kwon, 2008). Given these findings, it would appear that the most appropriate model of perfectionism is one in which the construct is both presented multidimensionally and grounded in a framework of intra- and interpersonal features.

In summary, perfectionism has been defined a number of ways, but appears to be characterized by striving for excessively high standards. Although prior theorists have considered the trait to be unidimensional, and largely maladaptive, current understandings of perfectionism view it as having both positive and negative qualities. Accordingly, prior researchers have postulated on what may discriminate adaptive, or normal, perfectionism from maladaptive, or neurotic, perfectionism, with self-discrepancy, self-criticism, and other factors proposed. Research has begun to consider the role of interpersonal elements in perfectionism, with evidence to suggest that the perceived source of high standards may be informative in discerning the trait's maladaptive or adaptive nature. Given this possibility, it is important to better understand how perfectionism comes to be destructive rather than desirable.

\section{Theories of Consequences of Perfectionism}

As suggested in the reviewed definitions, there are many facets of perfectionism that may make it particularly deleterious. Because perfectionistic individuals set or perceive excessively high standards, they will be more prone both to failing to meet these standards and to interpreting minor mistakes or shortcomings as failures (Burns, 1980; Frost \& Henderson, 1991; Frost et al., 1990). When these individuals also have overly critical self-evaluations, the self-discrepancy between standards and performance will be experienced as highly aversive. When additionally 
coupled with distorted cognitive processes, such as all-or-none thinking, catastrophizing, and selective attention to failure, this aversive experience will be potentiated and maintained. The individuals may then reengage in perfectionistic striving, in an effort to reduce self-discrepancy and its aversive effects; the destructiveness of perfectionism may thus self-perpetuate. Alternatively, individuals may fear failure to the extent that they attempt to avoid striving altogether. That is, perfectionism may not always manifest as a rigid, compulsive pursuit of high standards, but may sometimes manifest as an avoidance of goal pursuit entirely. Prior research lends support to this possibility, as perfectionism is associated with self-handicapping, such as procrastination (Martin et al., 1996; Neumeister, 2004; Saddler \& Sacks, 1993; Sheykhi, Fathabadi, \& Heidari, 2013; Sudler, 2014) and reduced effort after perceived failure (Hill, Hall, Duda, \& Appleton, 2011). Thus, whether associated with excessive striving or avoidance of striving, perfectionism, broadly defined, may confer risk for negative outcomes to the extent that the individual fails to meet standards and subsequently experiences aversive self-discrepancy. However, Hewitt and Flett's socially prescribed perfectionism may be uniquely concerning.

As previously mentioned, socially prescribed perfectionism appears to be more maladaptive than self-oriented perfectionism, both in degree and consistency of findings. Whereas self-oriented perfectionism intermittently exhibits modest associations with negative outcomes, socially prescribed perfectionism tends to maintain consistent, robust relations with these concerning effects. To this end, there appears to be something particularly maladaptive about socially prescribed perfectionism, even relative to the achievement striving found in selforiented perfectionism. This may be partially attributable to their different associations with variables theorized to maintain perfectionism. For instance, the relationship between perfectionism and procrastination is inconsistent in self-oriented perfectionism; whereas some 
studies find an association (Sudler, 2014), other studies do not (Mushquash \& Sherry, 2012;

Saddler \& Sacks, 1993; Steel, 2007) or find an inverse association (Martin et al., 1996), despite a consistent relationship between socially prescribed perfectionism and procrastination across these studies. Moreover, socially prescribed perfectionism, relative to self-oriented perfectionism, exhibits stronger relationships with overgeneralization, self-blame, avoidance, academic hassles, achievement hopelessness, lowered self-esteem, and fear of negative evaluations (Blankstein et al., 2007; Flett et al., 1991; Hewitt \& Flett, 1991b). In contrast, selforiented perfectionism is associated with greater self-efficacy and self-control (Flett et al., 1991; Martin et al., 1996). Thus, socially prescribed perfectionism may be more maladaptive, in part, because it is more closely associated with factors that are implicated in the maintenance of perfectionism, such as cognitive biases, self-criticism, and self-handicapping. However, there are additional pathways by which socially prescribed perfectionism confers risk for negative outcomes.

The reason that socially prescribed perfectionism is especially concerning may lie in its interpersonal nature. That is, when an individual perceives standards or expectations from a person other than the self, the anticipated consequences of not meeting these standards extend beyond failing to meet valued goals; instead, failure to meet standards may further be assumed to affect quality or stability of relationships with important others. In this way, perceived failure confers risk not only for personal disappointment, but also for loss of social support systems. Although understudied, this mechanism is supported by the finding that socially prescribed perfectionism is associated with beliefs that failure has interpersonal consequences, such as losing or upsetting important others (Conroy, Kaye, \& Fifer, 2007), whereas self- and otheroriented perfectionism did not exhibit this association. Thus, even when meeting standards, 
failure and its consequences may be more salient in socially prescribed perfectionism because of this anticipatory fear of losing social supports. Socially prescribed perfectionism may be additionally destructive for its association with interpersonal conflict.

Individuals with elevated socially prescribed perfectionism are not only highly sensitive to, but also incite, interpersonal problems (Mushquash \& Sherry, 2012). Building on this, the Perfectionism Social Disconnection Model (PSDM; Hewitt, Flett, Sherry, \& Caelian, 2006) proposes that individuals high in socially prescribed perfectionism may inadvertently deteriorate social connections, through processes such as hostile attributions or oversensitivity to rejection cues. The disruption of the social connection may then prompt the individual to attempt to present as perfect, in an effort to regain interpersonal acceptance and mend the relationship. However, this perfectionistic self-presentation may prevent the individual from authentic disclosure and connection. Supporting this model, socially prescribed perfectionism relates to social hopelessness, or feeling hopeless about one's relationships (Roxborough et al., 2012), to frequency of negative social interactions (Flett, Hewitt, Garshowitz, \& Martin, 1997) and to socially distant characteristics, social maladjustment, and distress (Hill, Zrull, \& Turlington, 1997). It likewise relates to perfectionistic self-presentation, including nondisplay and nondisclosure of imperfections (Chen, Hewitt, \& Flett, 2015; Mushquash \& Sherry, 2012). Selforiented perfectionism is less associated with these features (Chen et al., 2015; Mushquash \& Sherry, 2012; Roxborough et al., 2012) and is instead associated with appropriate assertiveness and adaptive interpersonal styles (Hill et al., 1997). In addition to these processes, motivation may also play a role in socially prescribed perfectionism's negative effects.

Beyond the interpersonal conflicts that may accompany socially prescribed perfectionism, this trait may also be destructive for its effect on motivation. That is, motivation 
in socially prescribed perfectionism is largely external; standards are not set by oneself but are instead perceived to be imposed by others. Indeed, socially prescribed perfectionism is negatively associated with intrinsic motivation and positively associated with extrinsic motivation, with specific motivation to display superior performance relative to others (Mills \& Blankstein, 2000). In contrast, self-oriented perfectionism is associated with intrinsic goal motivation (Mills \& Blankstein, 2000). Moreover, whereas self-oriented perfectionism is related to mastery goals, or desire to gain competence and proficiency relative to one's own prior ability, socially prescribed perfectionism is associated with performance-avoidance goals, or goals related to avoiding instances in which one's imperfections may be exposed, as well as performance-approach goals, or goals related to performance relative to peers (Neumeister \& Finch, 2006). Because of their extrinsic nature, the standards in socially prescribed perfectionism likely do not invigorate, excite, or challenge the individual, but instead pose as exhausting demands one must satisfy. Supporting this, socially prescribed perfectionism is associated with burnout and exhaustion and is inversely related to engagement, including vigor, dedication, and absorption (Childs \& Stoeber, 2010); self-oriented perfectionism exhibits the reverse relationship, relating to lower levels of burnout and exhaustion and to higher levels of engagement. Thus, motivation appears to be an additional mechanism by which socially prescribed perfectionism confers negative effects.

In sum, socially prescribed perfectionism may be particularly maladaptive, even when one is meeting perceived standards, because it is accompanied by an anticipatory fear of damaging social connections (Conroy et al., 2007), an increased frequency of negative social interactions (Flett et al., 1997; Hewitt et al., 2006; Mushquash \& Sherry, 2012), an isolating perfectionistic self-presentation (Chen et al., 2015; Mushquash \& Sherry, 2012), a motivation 
that is primarily external in nature (Mills \& Blankstein, 2000; Neumeister \& Finch, 2006), and an increased risk for burnout (Childs \& Stoeber, 2010). Self-oriented perfectionism does not tend to be characterized by these features. When individuals high in self-oriented perfectionism perform commensurate with their standards, their perfectionism is likely not a source of severe stress; they have little reason to anticipate or inadvertently incite loss of social support (Conroy et al., 2007; Flett et al., 1997; Hewitt et al., 2006; Mushquash \& Sherry, 2012), their perfectionism should not systematically disrupt authentic connection with others (Chen et al., 2015; Mushquash \& Sherry, 2012), they are striving towards personally valued goals (Mills \& Blankstein, 2000; Neumeister \& Finch, 2006), and they are more engaged and at a lower risk for burnout (Childs \& Stoeber, 2010). Less clear is the conditions under which the deleterious qualities of either dimension of perfectionism may be activated or exacerbated. That is, one's environment or life circumstances may alter the nature of perfectionism, such that both selforiented and socially prescribed perfectionism merit greater concern; a nascent, but neglected, body of research suggests negative life events, in particular, may moderate the effect of perfectionism on myriad negative outcomes. Likewise, perfectionism may reciprocally maintain or intensify the effects of negative life events. To this end, research has begun to investigate the interaction of negative life events with perfectionism. It is first worthwhile to examine how negative life events have been defined and conceptualized.

\section{Perfectionism in the Context of Negative Life Events}

Negative life events are theorized to be environmental conditions that catalyze stress processes (Linden, 1984; Rahe \& Arthur, 1978). Holmes and Rahe were perhaps the first to produce an empirically derived list of commonly experienced negative life events; they defined negative life events as those occurrences which necessitate noticeable change in adjustment and 
functioning (1967). Likewise, Myers, Lindenthal, Pepper, and Ostrander defined negative life events as occurrences which entail "role transformation, changes in status or environment, or impositions of pain" (1972, p. 399) and Sarason, Johnson, and Siegel defined negative life events by their undesirability and personal impact (1978). Brown theorized that life events were negative to the extent that they involve perception of threat (1974); Paykel similarly posited that a life event is negative insomuch as it is associated with distress (1974). Initial operationalizations of negative life events were derived from clinical experience and ranked by participants to reflect the anticipated adjustment required after such an event (Holmes \& Rahe, 1967). Subsequent operationalizations have built off these measures (Sarason et al., 1978), often informed by normative data (Linden, 1984; Tennant \& Andrews, 1976). Additionally, several measures have sought to include negative life events that may be relevant for particular populations, based on age (Liu, Liu, Yang, \& Zhao, 1997), life stage (Linden, 1984), and race (Utsey \& Ponterotto, 1996), among others. As definitions of negative life events have progressed, so too has the understanding of how these events affect relevant outcomes.

Research supports the destructive effect of negative life events on a range of outcomes. These events may relate to physical health, including severity of chronic illness, functional limitations, self-rated health status, mortality, and sudden cardiac death (Lantz, House, Mero, \& Williams, 2005; Rahe \& Lind, 1971; Wyler, Masuda, \& Holmes, 1971). Negative life events likewise affect mental health outcomes and correlates, including heavy drinking, drug use, anger, low self-esteem, depressive symptomatology, anxiety, hopelessness, presence and severity of NSSI, presence and severity of suicidal ideation, duration of acute suicidal crises, and odds of a suicide attempt (Bagge, Glenn, \& Lee, 2013; Covault et al., 2007; Dean et al., 1996; Flett et al., 1995; Joiner \& Rudd, 2000; Madge et al., 2011; O'Connor et al., 2010; Osvath, Vörös, \& Fekete, 
2004). Negative life events may discriminate those who ideate about NSSI and suicide attempts from those who engage in either behavior (Liu \& Tein, 2005; O'Connor, Rasmussen, \& Hawton, 2012). Alarmingly, recent negative life events predict prospective suicide attempts, even controlling for baseline diagnoses of borderline personality disorder, major depressive disorders, substance use disorders, and a history of childhood sexual abuse (Yen et al., 2005). On their own, negative life events are therefore related to myriad negative outcomes. As suggested, these relationships appear to be potentiated in individuals with elevated perfectionism.

Despite their concerning independent effects on physical and psychological health, negative life events may be experienced as more aversive for an individual with greater perfectionism. Researchers have primarily examined this moderating relationship as it relates to depression, distress, and, to a lesser extent, suicide. Related to depression and distress, prior research supports a relationship between negative life events and depressive symptomatology only for those high in perfectionism (Hewitt \& Dyck, 1986). Perfectionism appears to worsen the effects of negative life events on depressive symptomatology (Cheng, 2001; Lynd-Stevenson \& Hearne, 1999). There is some support for a directional pathway, with perfectionism and negative life events interacting to predict prospective distress, psychological adjustment, anxiety, and depression (Campos, Holden, Caçador, Fragata, \& Baleizão, 2018; Chang \& Rand, 2000; Flett et al., 1995; Hewitt et al., 1996; Joiner \& Schmidt, 1995; O'Connor et al., 2010). However, some research has failed to find an interaction between either dimension of perfectionism and negative life events in predicting these outcomes (Dean \& Range, 1999; Hewitt et al., 2002; Hewitt et al., 1996). Related to suicide, perfectionism and negative life events appear to elevate risk for both thoughts and behaviors. Research supports a potentiating effect of socially prescribed and selforiented perfectionism on the relationship between negative life events and suicidal ideation 
(Hewitt et al., 1994). Moreover, results of path analyses indicate that socially prescribed perfectionism moderates the relationship between negative life events and suicide behaviors, such that those with elevated perfectionism are at greater risk (Dean \& Range, 1996; Dean et al., 1996). Prospective studies lend support for a directional pathway, with socially prescribed perfectionism interacting with life stress to predict subsequent NSSI (O'Connor et al., 2010). However, as with depression and distress, other studies have found no significant interaction effect in predicting suicidal ideation (Dean \& Range, 1999). There are various reasons why perfectionism or negative life events may be especially concerning in the context of the other.

Perfectionism may become particularly maladaptive in the context of negative life events in part because of a general diathesis-stress framework (e.g. Chang \& Rand, 2000; Flett et al., 1995; O'Connor et al., 2010). That is, perfectionism may represent a form of vulnerability for outcomes such as depression and suicide that is activated by stressful life events (Hewitt \& Dyck, 1986; O'Connor, 2011). The mechanisms of such a relationship likely relate to various features of perfectionism; for instance, cognitive and attentional biases, such as overgeneralization, all-or-none thinking, and rigid thought processes (Burns, 1980), may lead an individual to evaluate a negative life event as more severe, persisting, or consequential than would someone with lower levels of perfectionism. Perfectionism is also associated with less effective coping strategies and lowered self-efficacy (Dunkley \& Blankstein, 2000; Sun, Gan, Liu, \& Chen, 2006), which may likewise deplete one's ability to manage negative life events. In addition, significant negative life events may disrupt an individual's ability to engage in achievement striving behavior (Flett et al., 1995), which may provoke extraneous anxiety in addition to that generated by the event itself. Moreover, individuals with elevated self-oriented or socially prescribed perfectionism are often characterized by a desire for control or a perceived 
lack of control, respectively (Flett et al., 1995; Hewitt \& Flett, 1991b); negative life events may thus thwart one's ability to feel in control or may confirm beliefs that one has little to no meaningful influence in life circumstances. However, in addition to these mechanisms, negative life events may potentiate the effects of perfectionism to the extent that one has concurrent perceptions of having failed.

\section{Negative Life Events as Perceived Failure}

Perhaps the most potent mechanism by which negative life events may interact with perfectionism is the appraisal of these events as failures. That is, the experience of failing should be increasingly aversive as one is increasingly perfectionistic; if negative life events are appraised as failures, they should be particularly distressing for individuals with elevated perfectionism, above and beyond the reasons already reviewed. Indeed, many studies investigating the interaction of negative life events with perfectionism operate under the assumption that these events signify a failure or shortcoming to individuals who are perfectionistic (e.g. Dean \& Range, 1996, 1999; Dean et al., 1996; Hewitt \& Flett, 1993; Hewitt et al., 1996). Hewitt, Flett, and Weber (1994, p. 449) write that perfectionists are "seen to view [life] stresses as failures." Dean, Range, and Goggin write of "stressful life events [that] fall short of the individual's standards and expectations" (1996, p. 182). However, the researchers' assumptions in employing negative life events in this capacity reveal what may be considered a missing factor; to date, there has been no known examination of how one's appraisal of a negative life event may partly explain this interaction effect. In other words, negative life events are inherently negative; losing a job or getting into a fight with a significant other would be likely to be experienced as unpleasant for almost anyone. Nonetheless, it appears that these events are experienced as even worse in individuals higher in perfectionism, and this may in part 
be attributable to the ways in which these individuals appraise the negative life events. That is, perfectionism may make one more prone to interpreting negative life events as failures. Whereas factors such as attentional biases, deficient coping, disrupted achievement striving, and perceived lack of control - among others — are aversive, perceived failure is perhaps most proximally related to perfectionism and may accordingly be most informative in explaining the interactive effect. Indeed, though empirically neglected, this postulation has received theoretical support; prior researchers have argued that it is not simply the number of life events, but how these events are interpreted, that elevates risk for suicidal ideation in perfectionism (Blatt, 1995; Dean \& Range, 1999). Despite the extensive research on appraisal style broadly, these processes have been severely understudied as they relate to perfectionism specifically. While a comprehensive review of cognitive appraisal is beyond the scope of this paper, it is nonetheless worthwhile to briefly define the concept before reviewing its relevance for perfectionism.

\section{The Role of Appraisal}

Throughout extant literature, appraisal is generally considered to be one's interpretation or evaluation of an event (Lazarus, 1966; Smith \& Lazarus, 1990). Appraisal, and reappraisal, may be considered forms of antecedent-focused emotion regulation, in that they target one's experiencing of an event, rather than just the external response to this internal experience (Gross, 1998). Researchers have proposed various domains which may be relevant for appraisal. For instance, one may make evaluations of the self, the world, and self-blame (Foa, Ehlers, Clark, Tolin, \& Orsillo, 1999). Related to an event, one may evaluate pleasantness, anticipated effort, novelty, attentional allocation, and locus of control and responsibility (Smith \& Ellsworth, 1985). Lazarus et al. (Lazarus, 1966; Lazarus \& Folkman, 1984; Smith \& Lazarus, 1993) delineate appraisals into primary appraisals, or how the event is perceived to relate to one's wellbeing, and 
secondary appraisals, or how the individual is perceived to be able to cope with the event. Primary appraisals are further delineated into motivational relevance, or the extent to which one is invested in the event, and motivational congruence, or the extent to which the event contributes to or detracts from personal goals. If the event is relevant and incongruent with goals, individuals may then engage in secondary appraisals, or evaluations of accountability, problemfocused coping potential, emotion-focused coping potential, and future expectancy (Lazarus, 1966; Lazarus \& Folkman, 1984; Smith \& Lazarus, 1990; Smith \& Lazarus, 1993). These secondary appraisals relate to assignment of responsibility, perceived ability to cope, and expectations for changes in one's current situation, respectively. The appraisals proposed by Lazarus et al. may be particularly relevant for the interpretation of a negative life event as a failure.

For a negative life event to be considered a failure, it would be relevant to the individual's goals and standards, it would be seen as counter to, or falling short of, these standards, and it would be appraised as being, at least partly, the individual's fault. If the event is irrelevant, it is unlikely that individuals would set, and thus be able to fall short of, standards. If the event is relevant, but congruent with standards, then the individual is performing commensurate with goals, and this event is less likely to be perceived as a failure. And if it is an event that is relevant and falls short of standards, but for which one nonetheless does not assume responsibility, this is less likely to be interpreted as a failure. Indeed, Lazarus and Smith argue that these appraisals collectively combine to predict certain emotional responses; one is likely to experience guilt, an emotion particularly germane to perfectionism, when an event is appraised as motivationally relevant and incongruent, and when one appraises the self, rather than others, 
as accountable (1990). Accordingly, how one appraises negative life events may have implications for their interaction with perfectionism.

Perfectionism may potentiate and maintain the effects of a negative life event to the extent that individuals view the event as a failure. In other words, for individuals higher in perfectionism, it may be more likely that the event is appraised as an occurrence relevant to the standards that have been set or imposed (e.g. motivational relevance; Smith \& Lazarus, 1990), as an instance of falling short of these standards (e.g. motivational congruence; Smith \& Lazarus, 1990), and as something the individual is responsible for (e.g. internal attribution, personal accountability; Abramson, Seligman, \& Teasdale, 1978; Smith \& Lazarus, 1990). To this end, fighting with a friend, for instance, may be experienced as particularly stressful to individuals with elevated perfectionism, in part because of the reasons reviewed (e.g. feeling a lack of control, struggling to identify effective coping responses), but primarily because the event is appraised as a failure — that is, because it is appraised as relevant to standards (e.g. to have relationships devoid of conflict), as falling short of standards, and as something for which one is responsible. Thus, perfectionism's interaction with negative life events likely operates along a pathway of appraisal of these events. Indeed, appraisal of these events as personal failures may also activate the other pernicious features of perfectionism (e.g. ineffective coping), further explaining the pathway to suicide risk and other maladaptive outcomes. As understandings of these processes become increasingly clear, it becomes increasingly important to elucidate their underlying mechanisms, so that prevention and intervention efforts may be appropriately tailored. 


\section{The Pathway to Risk for Suicide}

As reviewed, perfectionism exhibits a robust relationship with suicide intent (Hewitt, Flett, \& Turnbull-Donovan, 1992), suicidal ideation (Beevers \& Miller, 2004; Dean et al., 1996; Hewitt et al., 2014; Hewitt et al., 1994; Jacobs et al., 2009; Smith, Speth, et al., 2017), and suicidal motivation, suicide preparation, and occurrence, severity, and number of lifetime suicide attempts (Flamenbaum \& Holden, 2007; Hewitt et al., 1998; Smith, Sherry, et al., 2018). Negative life events likewise elevate risk for suicide ideation (Dean \& Range, 1999; Hirsch, Wolford, LaLonde, Brunk, \& Parker-Morris, 2009), suicide attempts (Liu \& Tein, 2005; O'Connor et al., 2012; Yen et al., 2005) and intensity and duration of suicidal crises (Joiner \& Rudd, 2000). Similarly, perceptions of failing have been implicated theoretically (Baumeister, 1990; Domino, 2005) and empirically to suicide ideation (Chatard \& Selimbegović, 2011; Tang, Wu, \& Miao, 2013) and risk for an attempt (Bulik, Carpenter, Kupfer, \& Frank, 1990; Gould, Fisher, Parides, Flory, \& Shaffer, 1996; Richardson, Bergen, Martin, Roeger, \& Allison, 2005). However, to the extent that there may be intermediary variables along the pathway to risk, examination of the direct effects is insufficient; effective amelioration of risk requires knowledge of, and intervention in, those variables that precede acute risk. Accordingly, research has begun to consider the mechanisms by which perfectionism, negative life events, and appraisal confer risk for suicide.

Whereas recognizing the relationship of, collectively, perfectionism, negative life events, and appraisal with risk for suicide is informative, examination of the direct effect in isolation misses valuable information on the processes by which these variables confer risk. That is, there are likely intervening variables, and these may serve as important indicators of progression of risk. Knowledge of such mediators may allow for more specified assessment, monitoring, and 
intervention regarding preventing suicidal thoughts and behaviors in relevant individuals. Prior researchers have begun to propose mechanisms by which these variables may generate maladaptive outcomes. For instance, theories of consequences of perfectionism refer to its association with cognitive distortions (Burns, 1980), self-handicapping (Hill et al., 2011; Martin et al., 1996; Neumeister, 2004; Saddler \& Sacks, 1993; Sheykhi et al., 2013; Sudler, 2014), belief that failure has interpersonal consequences (Conroy et al., 2007), social hopelessness (Roxborough et al., 2012), frequency of negative social interactions (Flett et al., 1997), perfectionistic self-presentation (Chen et al., 2015; Mushquash \& Sherry, 2012), extrinsic motivation (Mills \& Blankstein, 2000), and burnout and exhaustion (Childs \& Stoeber, 2010), among other consequences. Whereas these factors may explain why perfectionism, and particularly socially prescribed perfectionism, is maladaptive, they do not have specificity in explaining perfectionism's relationship with suicide. In other words, extrinsic motivation or frequency of negative social interactions may comprise perfectionism's negative qualities, but it is unclear if these would bridge the gap between perfectionism and suicide. Instead, these pernicious features of perfectionism may lead the individual to an affective state which in turn predicts risk for suicide. Likewise, negative life events and appraisal of these events as failures may generate unbearable distress, with this distress, rather than the events or appraisals themselves, more proximally predicting risk for suicide. The Cry of Pain model of suicide (Williams, 2001) may be particularly well-suited to clarifying this pathway and identifying the intermediate factors implicated in the progression to suicide risk.

\section{The Cry of Pain Model of Suicide}

The Cry of Pain model of suicide (Williams, 2001) may offer a compelling framework by which to understand the association of perfectionism, negative life events, appraisal of these 
events, and risk for suicide. Williams's model is an escape theory of suicide, which built off of that proposed by Baumeister (1990) and developed from a general diathesis-stress perspective (e.g. Alloy et al., 1999; O'Connor \& Sheehy, 2000). Additionally, it bears similarity to the "arrested flight" model in animal studies which invokes the role of social rank variables, such as perceived inferiority, submission, helplessness, and passivity (Gilbert \& Allan, 1998). Broadly, this model predicts that individuals develop risk for suicide when they encounter excessively stressful events or life circumstances, when they feel defeated by these circumstances, and when they perceive little chance of rescue from external sources of support (Williams, 2001; Williams $\&$ Pollock, 2008). Despite its succinctness, a growing body of research supports the usefulness of this model. These factors discriminate suicidal individuals from hospital controls, above and beyond depression and hopelessness (O'Connor, 2003); they likewise distinguish first-time from repeat self-harm patients and both groups from hospital controls (Rasmussen et al., 2010). Supporting its independent relationship with suicide risk, defeat is associated with suicidal ideation, above and beyond the effects of depression and hopelessness (Gilbert \& Allan, 1998; Taylor, Gooding, Wood, \& Tarrier, 2011; Taylor, Wood, Gooding, \& Tarrier, 2010). Moreover, defeat predicts prospective suicidal ideation, controlling for baseline ideation, posttraumatic stress disorder severity, depressive symptoms, and hopelessness (Panagioti, Gooding, \& Tarrier, 2015). Thus, Williams's Cry of Pain model may offer a concise and specific framework within which to conceptualize suicide risk. However, the use of defeat as a proximal risk factor for suicide may be somewhat inconsistent with the data. That is, defeat appears to be more accurately understood as a stable personality trait (e.g. 1-year test-rest $r=.90$; Griffiths, Wood, Maltby, Taylor, \& Tai, 2014), rather than the acutely aversive, fluctuating emotional state described in the model (Williams, 2001). William's model may be better represented by the 
conceptually similar, more mutable construct of demoralization. Demoralization is considered to be state-like - amenable to change corresponding to shifts in one's internal or external environment (Clarke \& Kissane, 2002; Frank, 1974; Frank \& Frank, 1993; Kissane, Levin, Hales, Lo, \& Rodin, 2011; Kissane, Wein, Love, \& Lee, 2004). Supporting this, demoralization exhibits expected reductions after interventions (Griffith \& Gaby, 2005; Marsa, Bahmani, Naghiyaee, \& Barekati, 2017). Demoralization is associated with, but distinct from, depressive symptoms, hopelessness, poorer quality of life, and attenuated positive future expectancies (Clarke \& Kissane, 2002; Kissane et al., 2004; Lee et al., 2012; Mehnert, Vehling, Höcker, Lehmann, \& Koch, 2011; Rafanelli et al., 2013; Tang, Wang, \& Chou, 2015). Because of the severity of the state, it is theorized to be more proximal to, and specific in predicting, suicide risk, relative to these more pervasive or stable factors (Clarke \& Kissane, 2002) and empirical work supports this postulation (Fang et al., 2014; Jacobsen et al., 2006). In sum, whereas Williams theorized that individuals may be suicidal to the extent that they feel defeated, the similar but distinct state of demoralization may better approximate the unbearable distress that precedes elevated suicide risk.

\section{Demoralization}

Demoralization is broadly defined as a sense of learned helplessness and existential anguish (Kissane, 2004). Specifically, it is characterized by a perceived failure to effectively cope with actual or appraised stressors, as well as dysphoria, disheartenment, despair, dejection, perceived inefficacy, and isolation (Frank, 1974; Kissane et al., 2004). Indeed, demoralization is described as a state "in which meaningless predominates and from which profound hopelessness and desire to die may result" (Kissane, 2004, p. 23). In a state of acute demoralization, "life is viewed as pointless and worthless, with no hope for future fulfillment;" the individual feels both 
helpless and trapped by this aversive experience (Kissane, 2004, p. 27). Accordingly, demoralization has been linked qualitatively (Clarke \& Kissane, 2002; Kissane, Clarke, \& Street, 2001) and empirically (Anestis et al., 2018; Fang et al., 2014; Kissane et al., 2004; Lau, Morse, \& Macfarlane, 2010; Vehling et al., 2017) to suicidal ideation, as well as empirically to clinicianrated suicide risk (Anestis et al., 2018). To the extent that demoralization resembles an acute state of defeat, Williams's model may offer a useful perspective from which to conceptualize the association of perfectionism, negative life events, appraisal, and suicide risk. Importantly, there is reason to believe that these variables would relate to demoralization.

Even in the absence of negative life events, perfectionism may relate to demoralization, with both broad and specific support for this pathway. Broadly, socially prescribed perfectionism appears to lower stress tolerance, such that, even at low levels of acute life stress, it is associated with self-harm (O'Connor et al., 2010), suggesting that it does not require the presence of stressors to activate these maladaptive outcomes. Accordingly, socially prescribed perfectionism could conceivably relate to demoralization even without moderating factors such as negative life events. Additionally, and as reviewed earlier, perfectionism exhibits a main effect with similar, but distinct outcomes, such as depression (e.g. Bieling et al., 2004; Enns \& Cox, 2005; Sassaroli et al., 2008). Despite the robust literature on perfectionism and various negative outcomes broadly, the specific pathway from perfectionism to demoralization has been severely neglected. Two known studies have examined perfectionism's association with defeat, finding support for this relationship (Wetherall, Robb, \& O'Connor, 2018; Wyatt \& Gilbert, 1998). No known studies have examined if perfectionism relates to demoralization. Whereas the association of perfectionism and defeat is informative, its predictive utility is limited, to the extent that it likely concerns the static relationship of two relatively stable personality constructs. Such findings do 
not provide insight on the role perfectionism may play as a vulnerability factor for subsequent fluctuations in demoralization, particularly in the context of certain life circumstances. Despite a lack of empirical investigation, early theoretical conceptualizations of demoralization considered it to stem directly from perceptions of failing to meet one's own or others' expectations (Frank, 1974). Thus, as individuals set or intuit increasingly high standards that are increasingly discrepant with their actual or perceived abilities, they may be increasingly prone to these experiences of demoralization. In other words, individuals who are highly perfectionistic will likely not be able to sustainably and consistently meet unrealistically high standards; in those instances in which they fall short, they may experience the extreme distress, helplessness, inefficacy, dejection, and despair characteristic of demoralization. Broadly then, socially prescribed perfectionism in particular may be associated with demoralization as a main effect simply because the expectations are perceived as overwhelming and impossible to meet, while failure is simultaneously viewed as conferring severe consequences (e.g. Conroy et al., 2007). The individual is trapped between, on the one hand, burnout (Childs \& Stoeber, 2010) and striving to meet seemingly unachievable standards, and, on the other hand, the implications of relinquishing standards and potentially deteriorating social support and disappointing close others (Conroy et al., 2007). The individual may begin to feel demoralized and hopeless in the face of this nonchoice and may accordingly view suicide as an escape. Furthermore, individuals who are perfectionistic may be particularly prone to feeling as though they are failing to effectively cope, a key feature of demoralization, both because of actual deficits in coping (Flett, Hewitt, Blankstein, et al., 1996) and because of perfectionism's cognitive biases (e.g. selective attention to failure; Burns, 1980). In this way, the weight of expectations, the fear of consequences of failure, the need to present as perfect, the burnout and exhaustion, and the 
coping deficits inherent in perfectionism may all increase feelings of demoralization. Negative life events may operate similarly in their relationship with demoralization.

As with the literature on perfectionism, there is a relatively sizeable amount of support for a broad relationship between negative life events and maladaptive outcomes and a smaller body of research to support the specific pathway to demoralization. For instance, like perfectionism and as reviewed earlier, a growing body of research associates negative life events with similar constructs such as depression (e.g. Cheng, 2001; Hewitt \& Dyck, 1986). Nonetheless, there is some extant empirical work supporting the relationship of cumulative negative life events with demoralization specifically. Namely, the experience of a recent stressful life event predicts greater demoralization (Evans, Jacobs, Dooley, \& Catalano, 1987) and the severity of demoralization appears to increase linearly as one endorses a greater number of negative life events (Tein, Sandler, \& Zautra, 2000). Additional research indicates that stressful life events relate to demoralization, insomuch as that they are associated with negative change (Lennon, Dohrenwend, Zautra, \& Marbach, 1990), and the experience of one or more recent life events that feels uncontrollable and disruptive relates to demoralization (Shrout et al., 1989). Conceptually, negative life events may relate to perceptions of demoralization to the extent that they catalyze stress processes (Linden, 1984; Rahe \& Arthur, 1978) or incite pain (Myers et al., 1972) or distress (Paykel, 1974). As one experiences a greater number of negative life events, one may begin to question the existence of a "caring world," or a world in which exists a level of protection, security, and freedom from harm (Janoff-Bulman \& Morgan, 1994, p. 58) Accordingly, individuals may feel demoralized by the apparent lack of protection from these events. Moreover, as the experience of negative life events accumulates, individuals may feel increasingly disheartened, hopeless, helpless, and dejected; may increasingly perceive 
themselves as unable to cope with these events; and may increasingly view life as unpredictable and meaningless, all defining features of demoralization. When individuals simultaneously experience high levels of perfectionism and appraisal of these events as personal failures, it is this much more likely that they will face perceptions of demoralization.

On their own, perfectionism, particularly socially prescribed perfectionism, and negative life events may elevate one's risk for feelings of demoralization. However, it is likely that their respective effects are potentiated in the context of one another and that this interaction itself is partly attributable to the interpretation of negative life events as personal failures. That is, as individuals high in perfectionism strive to balance their exhaustion from goal pursuit (Childs \& Stoeber, 2010) with their fear of consequences of failing to meet goals (Conroy et al., 2007), the experience of negative life events interpreted as failures may thwart any sense of control the individual has of being able to maintain a semblance of perfection and balance. Accordingly, these individuals are not only demoralized by this nonchoice, and by the already severe effects of negative life events, but now also by the prospect of failing despite their valuing of perfection. These negative life events appraised as failures may undermine any sense of self-efficacy or empowerment, of which perfectionism may give the illusion, and may instead foster a sense of helplessness to meet standards. For individuals who desire a high level of control in their lives (Flett et al., 1995; Hewitt \& Flett, 1991b), the occurrence of negative life events appraised as failures may feel unbearably discouraging; despite pursuing these high standards, whether for inter- or intrapersonal reasons, the individual has failed to manifest perfection and has instead encountered failure. Thus, as theoretically postulated (Frank, 1974; O'Connor, 2011; Williams, 2001) and as supported in preliminary research (Evans et al., 1987; Lennon et al., 1990; Shrout et al., 1989; Tein et al., 2000; Wetherall et al., 2018; Wyatt \& Gilbert, 1998), demoralization may 
be particularly germane for understanding perfectionism's maladaptive nature, especially in the presence of negative life events, to the extent that these events are appraised as failures.

\section{An Integrated Model of the Contextual Effects of Perfectionism}

As indicated by the conceptual model in Figure 1, perfectionism, negative life events, and appraisal of those events as personal failures may collectively relate to feelings of demoralization. The association of perfectionism with demoralization has been severely understudied; nonetheless, preliminary research supports a relationship with the similar construct of defeat (Wetherall et al., 2018; Wyatt \& Gilbert, 1998). There is reason to believe that perfectionism, and socially prescribed perfectionism in particular, may relate to demoralization specifically. To the extent that such individuals are caught between exhaustive achievement striving (e.g. Childs \& Stoeber, 2010), on the one hand, and anticipation of severely negative consequences of failure (e.g. Blankstein et al., 2007; Conroy et al., 2007), on the other, they will likely feel the entrapment, anguish, despair, and dejection characteristic of demoralization. Whereas individuals with elevated self-oriented perfectionism may fear and strive to avoid failure and may anticipate some aversive intrapersonal consequences (e.g. disappointment with self), those with elevated socially prescribed perfectionism are additionally burdened by their concern for interpersonal consequences of failure (e.g. being devalued by close others, loss of social support; Conroy et al., 2007). To this end, socially prescribed perfectionism may exhibit a greater relationship with demoralization. Furthermore, socially prescribed perfectionism may more closely relate to demoralization due to the cognitive biases and actual coping deficits associated with this dimension of perfectionism (Dunkley \& Blankstein, 2000; Flett, Hewitt, Blankstein, et al., 1996; Sun et al., 2006) which may relate to the perceived inability to cope inherent in demoralization. 
As suggested in Figure 1, the relationship of these dimensions of perfectionism to demoralization may be potentiated as the individual experiences a greater number of negative life events. Extant research supports the pathway from negative life events to demoralization as a main effect (Lennon et al., 1990; Shrout et al., 1989; Tein et al., 2000), and there is reason to believe that these events would augment the main effects of perfectionism. In individuals with elevated perfectionism, these negative life events may tax already depleted coping mechanisms (Flett, Hewitt, Blankstein, et al., 1996), may be perceived as more severe or enduring, may cause additional anxiety or distress secondary to disruption of achievement striving (Flett et al., 1995), and may thwart a highly valued sense of control (Flett et al., 1995; Hewitt \& Flett, 1991b). Importantly, however, and as reflected in the model, negative life events may interact with perfectionism primarily through a pathway of appraising these events as personal failure. It is likely that events appraised in this manner will be both increasingly distressing and increasingly likely as individuals exhibit increasingly elevated levels of perfectionism. Not only does the individual face the alternative mechanisms of the interaction of perfectionism and negative life events (e.g. disruption of achievement striving, thwarted sense of control), but must also face the possibility of perceived failure despite desiring perfection. Even when performing commensurate with standards, perfectionism appears to be deleterious; it is likely this is much more so in the context of perceptions of falling short of standards. This has yet to be adequately empirically tested.

In summary, there is conceptual and, in some instances, preliminary empirical support to suggest that perfectionism, negative life events, and appraisals of personal failure would lead to an acute state of demoralization. Given that demoralization has been linked theoretically and empirically to suicide risk (Anestis et al., 2018; Fang et al., 2014; Kissane et al., 2004; Lau et al., 
2010; Vehling et al., 2017), determining the degree to which these vulnerabilities relate to demoralization, and in which contexts, may inform more targeted prevention and intervention efforts. Therefore, the present study seeks to integrate this existing research, with the objective of conceptualizing and testing a more comprehensive model by which perfectionism relates to a state of elevated risk.

\section{Objectives of the Present Study}

The present study seeks to examine the relationship between perfectionism and demoralization, to evaluate a potential mechanism by which perfectionism may come to be distally associated with suicidal ideation. Drawing from extant conceptualizations of perfectionism (Hewitt \& Flett, 1991b) and theories of consequences of perfectionism (Burns, 1980; Frost \& Henderson, 1991; Frost et al., 1990), this study extends prior work by proposing a more novel, and potentially more informative, pathway and by examining the influence of certain life circumstances on this relationship. The study likewise incorporates theories of appraisal (Smith \& Lazarus, 1990; Smith, Haynes, Lazarus, \& Pope, 1993), to consider and test the role that cognitive factors may play in moderating these processes. Whereas prior literature has argued that perfectionism may be particularly destructive in the context of negative life events to the extent that these events are proxies for failing (Dean \& Range, 1996, 1999; Dean et al., 1996; Hewitt \& Flett, 1993; Hewitt et al., 1996), there is no known empirical investigation of how relevant appraisals (i.e. appraising the event as a personal failure) mediate the effect of negative life events on the relationship between perfectionism and negative outcomes. Given that appraisals may be more easily changed than the occurrence of negative life events, knowing the degree to which they explain this interaction will be integral in determining their utility as a point of intervention. That is, if negative life events interact with perfectionism through a pathway of 

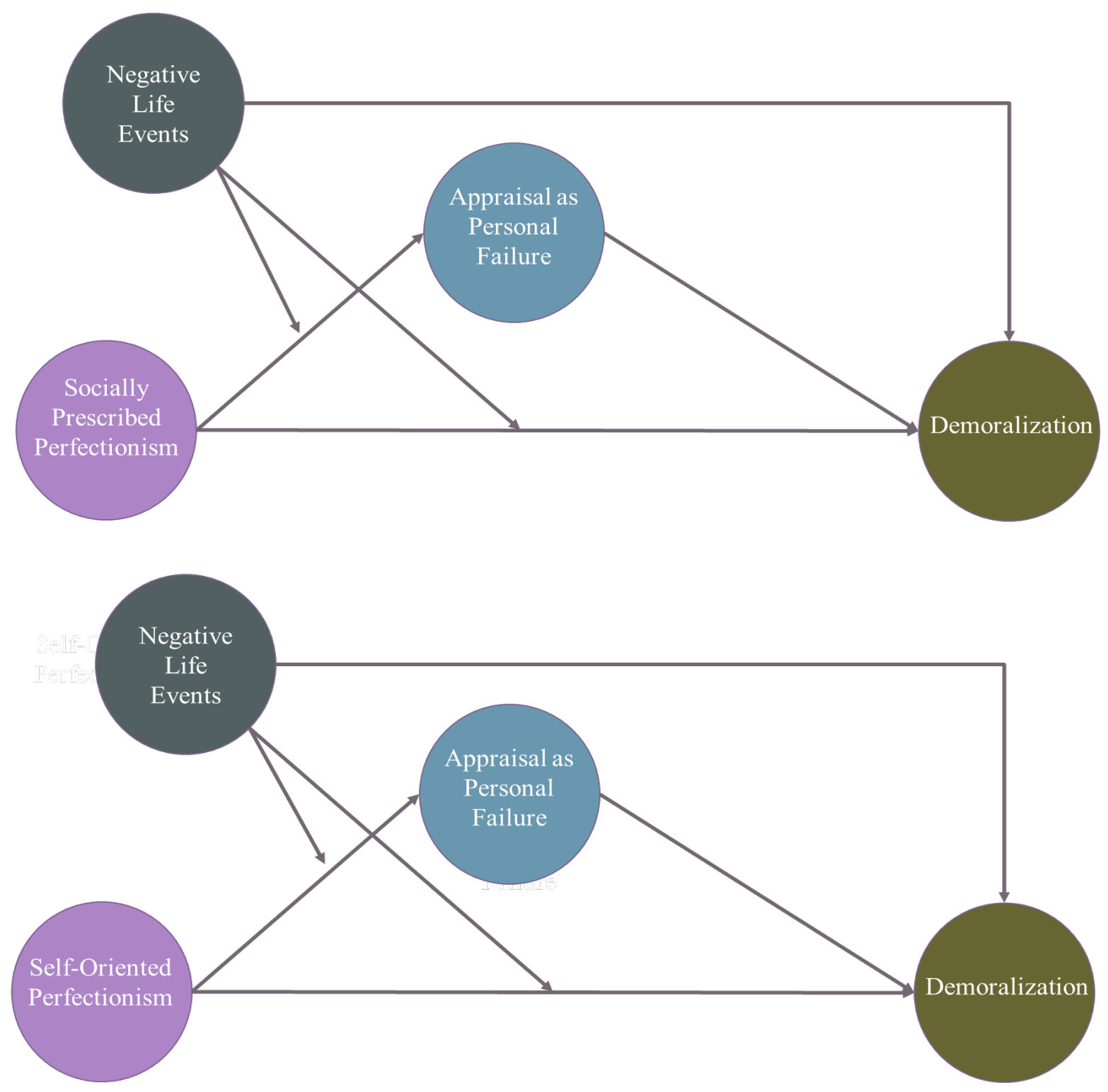

Figure 1. Hypothesized model of the relationship between perfectionism (predictor) and demoralization (dependent variable), in the context of negative life events (moderator) and through a pathway of appraisal as personal failures (mediator). 
appraisal as failures, reappraisal may be protective for such individuals. This investigation will operate within a defeat framework of suicide (Williams, 2001). There appears to be extensive theoretical support for such a model (e.g. Frank, 1974; O'Connor, 2011), but there is a dearth of integrated empirical research. The few studies that have been conducted have failed to account for these cognitive and environmental moderators and have examined perfectionism in relation to defeat (Wetherall et al., 2018; Wyatt \& Gilbert, 1998). Defeat appears to be a relatively stable trait (Griffiths et al., 2014) and may therefore serve little utility in predicting acute fluctuations in suicide risk. To this end, the present study seeks to test a related construct, demoralization, which may better approximate experiences of acute defeat and which may be particularly relevant to the features of perfectionism, negative life events, and appraisals.

Given that perfectionism is both pervasive (Grzegorek et al., 2004; Herman et al., 2011; Rice et al., 2011) and steadily increasing in prevalence (Curran \& Hill, 2017; Flett \& Hewitt, 2014) and given that it is robustly associated with myriad negative correlates and outcomes (e.g. Hamilton \& Schweitzer, 2000; Hewitt et al., 1994; O'Connor et al., 2010; Rasmussen et al., 2008), it is imperative to better identify both the mechanisms by which it effects these undesirable consequences and the factors that may potentiate or ameliorate its pernicious qualities. Accordingly, the present study seeks to propose and test an integrated model of the maladaptive nature of perfectionism.

The present study includes the following aims and specific hypotheses:

Aim 1.) Determine whether perfectionism alone may confer risk for demoralization. A better understanding of perfectionism's main effects will help elucidate whether its maladaptive nature requires the presence of perceived failure, or if it may be activated even while individuals perceive themselves as performing commensurate with standards. 
Hypothesis 1A. It is hypothesized that socially prescribed perfectionism will relate to feelings of demoralization as a main effect and independent from co-occurring self-oriented perfectionism.

Hypothesis 1B. It is hypothesized that self-oriented perfectionism will relate to feelings of demoralization as a main effect and independent from co-occurring socially prescribed perfectionism.

Hypothesis 1C. It is hypothesized that the unique main effect of socially prescribed perfectionism on feelings of demoralization will be significantly greater than the unique main effect of self-oriented perfectionism on feelings of demoralization.

Aim 2.) Document the relationship between negative life events and demoralization. Evaluate the degree to which life circumstances activate or potentiate the maladaptive features of perfectionism as related to demoralization. Understanding the contexts in which perfectionism may be particularly destructive will allow for more targeted identification of risk and implementation of intervention.

Hypothesis 2A. It is hypothesized that as individuals experience an increasing number of negative life events, they will experience increasingly greater feelings of demoralization as a main effect.

Hypothesis 2B. It is hypothesized that socially prescribed perfectionism will increasingly relate to demoralization as individuals experience a greater number of negative life events.

Hypothesis 2C. It is hypothesized that self-oriented perfectionism will increasingly relate to demoralization as individuals experience a greater number of negative life events. 
Aim 3.) Examine the role of appraisal in mediating the interaction of perfectionism and negative life events. By considering the influence of appraisal in these processes, it may be possible to identify the pathway by which negative life events interact with perfectionism, and thus identify points of intervention which may ameliorate or attenuate the effects of this interaction. If perfectionism is particularly maladaptive for individuals who experience greater negative life events because these events are appraised as personal failures, it may be possible to attend to and challenge these appraisals to reduce the effects of this interaction.

Hypothesis 3A. It is hypothesized that the interactive effect of socially prescribed perfectionism and negative life events on demoralization will operate in part through a pathway of appraisal. In the context of negative life events, individuals who have increasingly elevated socially prescribed perfectionism will increasingly appraise these negative life events as personal failures and will accordingly feel increasingly demoralized.

Hypothesis 3A (i). It is hypothesized that as individuals are increasingly elevated in socially prescribed perfectionism, they will increasingly appraise negative life events as personal failures as a main effect.

Hypothesis 3A (ii). It is hypothesized that socially prescribed perfectionism will increasingly relate to appraisal as failure as individuals experience a greater number of negative life events.

Hypothesis 3A (iii). It is hypothesized that as individuals increasingly appraise negative life events as personal failures they will feel increasingly demoralized. 
Hypothesis 3B. It is hypothesized that the interactive effect of self-oriented perfectionism and negative life events on demoralization will operate in part through a pathway of appraisal. In the context of negative life events, individuals who have increasingly elevated self-oriented perfectionism will increasingly appraise these negative life events as personal failures and will accordingly feel increasingly demoralized.

Hypothesis 3B (i). It is hypothesized that as individuals are increasingly elevated in self-oriented perfectionism, they will increasingly appraise negative life events as personal failures as a main effect.

Hypothesis 3B (ii). It is hypothesized that self-oriented perfectionism will increasingly relate to appraisal as failure as individuals experience a greater number of negative life events.

Hypothesis 3B (iii). It is hypothesized that as individuals increasingly appraise negative life events as personal failures they will feel increasingly demoralized. 


\section{Methods}

\section{Participants}

364 participants were recruited via the University of South Florida psychology department's online SONA participant pool. Sample size was determined with consideration to calculations of statistical power using G*POWER 3.1.9.2. Power analysis was based on the ability to detect the additional variance accounted for by the proposed two-way interaction term. The power analysis indicated that a sample size of 199 would be needed to detect a small to medium effect $\left(\Delta R^{2}=.04\right)$ with power $=.80$ and $\alpha=.05$. A total of 320 participants passed attention check tests, so this sample was used in all analyses.

Inclusion criteria consisted of any individuals who were at least 18 years of age, enrolled in a psychology course, currently enrolled as part- or full-time undergraduate students, and able to read and comprehend English written language fluently, to ensure accurate completion of the study measures. No other exclusion criteria were applied. Participants were not financially compensated for participation but received one point of extra credit per half hour of participation or as indicated by respective instructor policies.

The included participants had a mean age of $21.18(S D=4.10)$ and were primarily female $(81.6 \%)$ and heterosexual $(82.8 \%)$. The sample was relatively diverse with regards to race and ethnicity, with $44.7 \%$ of participants identifying as Caucasian, $14.4 \%$ identifying as Hispanic or Latino, 9.7\% identifying as Asian or Asian American, 8.4\% identifying as Black or African American, 3.8\% identifying as Arabic or Middle Eastern, 2.8\% identifying as another race or ethnicity, and the remaining $16.2 \%$ of participants identifying as multiracial. Approximately $28 \%$ 
of the participants were first-generation college students; $24.1 \%$ were freshmen, $25.0 \%$ were sophomores, $26.6 \%$ were juniors, $19.6 \%$ were seniors, and $4.7 \%$ were in their fifth year of college or greater. Participants primarily reported living in an off-campus house or apartment $(42.5 \%)$, in an on-campus residence hall $(28.4 \%)$, or at home with family $(28.1 \%)$. See Table 1 for more detailed information on demographics in the present sample.

Table 1. Sample Demographics.

\begin{tabular}{|c|c|c|c|c|c|c|}
\hline$\underline{\text { Variable }}$ & & $\underline{N(\%)}$ & $\underline{\text { Mean }}$ & $\underline{\mathrm{SD}}$ & $\underline{\text { Skewness }}$ & $\underline{\text { Kurtosis }}$ \\
\hline Age & & $319(100 \%)$ & 21.18 & 4.10 & 3.73 & 18.07 \\
\hline \multirow[t]{3}{*}{$\underline{\text { Gender }}$} & Female & $261(82 \%)$ & & & & \\
\hline & Male & $56(18 \%)$ & & & & \\
\hline & Neither & $3(0.9 \%)$ & & & & \\
\hline \multirow[t]{2}{*}{$\underline{\text { Transgender }}$} & No & $315(98 \%)$ & & & & \\
\hline & Yes & $5(1.6 \%)$ & & & & \\
\hline \multicolumn{7}{|l|}{ Sexual } \\
\hline \multirow[t]{5}{*}{ Orientation } & Heterosexual & $265(83 \%)$ & & & & \\
\hline & Bisexual & $35(11 \%)$ & & & & \\
\hline & Gay/Lesbian & $11(3.4 \%)$ & & & & \\
\hline & Asexual & $1(0.3 \%)$ & & & & \\
\hline & Other & $8(2.5 \%)$ & & & & \\
\hline
\end{tabular}


Table 1. (Continued)

\begin{tabular}{|c|c|c|c|c|c|c|}
\hline Variable & & $\underline{N(\%)}$ & Mean & $\underline{\mathrm{SD}}$ & $\underline{\text { Skewness }}$ & $\underline{\text { Kurtosis }}$ \\
\hline \multirow[t]{7}{*}{ Race/Ethnicity } & Caucasian & $143(44 \%)$ & & & & \\
\hline & Hispanic/Latinx & $46(14 \%)$ & & & & \\
\hline & $\begin{array}{l}\text { Asian/Asian- } \\
\text { American }\end{array}$ & $31(9.7 \%)$ & & & & \\
\hline & $\begin{array}{c}\text { Black/ } \\
\text { African- } \\
\text { American }\end{array}$ & $27(8.4 \%)$ & & & & \\
\hline & $\begin{array}{l}\text { Arabic/Middle } \\
\text { Eastern }\end{array}$ & $12(3.8 \%)$ & & & & \\
\hline & More than 1 race & $51(16 \%)$ & & & & \\
\hline & Other & $9(2.8 \%)$ & & & & \\
\hline \multicolumn{7}{|l|}{$\begin{array}{l}\text { First- } \\
\text { Generation }\end{array}$} \\
\hline \multirow[t]{2}{*}{ College Student } & No & $230(72 \%)$ & & & & \\
\hline & Yes & $90(28 \%)$ & & & & \\
\hline \multirow[t]{5}{*}{ Year } & Freshman & $76(24 \%)$ & & & & \\
\hline & Sophomore & $79(25 \%)$ & & & & \\
\hline & Junior & $84(26 \%)$ & & & & \\
\hline & Senior & $62(19 \%)$ & & & & \\
\hline & Year 5+ & $15(4.7 \%)$ & & & & \\
\hline Living & Off-Campus & & & & & \\
\hline \multirow[t]{5}{*}{ Arrangement } & $\begin{array}{l}\text { House or } \\
\text { Apartment }\end{array}$ & $136(43 \%)$ & & & & \\
\hline & $\begin{array}{c}\text { On-Campus } \\
\text { Residence Hall }\end{array}$ & $91(28 \%)$ & & & & \\
\hline & $\begin{array}{l}\text { At Home with } \\
\text { Family }\end{array}$ & $90(28 \%)$ & & & & \\
\hline & $\begin{array}{l}\text { Fraternity or } \\
\text { Sorority }\end{array}$ & $2(0.6 \%)$ & & & & \\
\hline & Other & $1(0.3 \%)$ & & & & \\
\hline
\end{tabular}




\section{Table 1. (Continued)}

\begin{tabular}{|c|c|c|c|c|c|c|}
\hline$\underline{\text { Variable }}$ & & $\mathrm{N}(\%)$ & $\underline{\text { Mean }}$ & $\underline{\mathrm{SD}}$ & $\underline{\text { Skewness }}$ & $\underline{\text { Kurtosis }}$ \\
\hline \multicolumn{6}{|l|}{ Marital and } & Relationship \\
\hline \multirow[t]{6}{*}{$\underline{\text { Status }}$} & Single & $180(56 \%)$ & & & & \\
\hline & $\begin{array}{c}\text { In Relationship } \\
\text { and Not Living } \\
\text { with Partner }\end{array}$ & $98(31 \%)$ & & & & \\
\hline & $\begin{array}{l}\text { In Relationship } \\
\text { and Living with } \\
\text { Partner }\end{array}$ & $32(10 \%)$ & & & & \\
\hline & Married & $8(2.5 \%)$ & & & & \\
\hline & Widowed & $1(0.3 \%)$ & & & & \\
\hline & Other & $1(0.3 \%)$ & & & & \\
\hline
\end{tabular}

\section{Measures}

Demographic Questionnaire: The present study used a demographic assessment measure (see Appendix A) to collect basic demographic data, including age, gender, year in undergraduate course of study, sexual identity, race/ethnicity, language(s) spoken, current living arrangement (e.g. on-campus, off-campus apartment, etc.), marital status, and employment status. This measure took approximately five minutes to complete.

Demoralization Scale; DS (Appendix B; Kissane et al., 2004): The DS is a 24-item selfreport measure of feelings of state demoralization, which took approximately five minutes to complete. Respondents used a 5-point Likert scale ranging from 0, "Never," to 4, "All the Time," to indicate how often in the previous two weeks they felt various features of demoralization. The scale produces five subscales, measuring "Dysphoria," "Loss of meaning and purpose," "Disheartenment," "Helplessness," and "Sense of failure." Example items from each scale are, 
respectively, "I have a lot of regret about my life," "My life seems to be pointless," "I feel trapped by what is happening to me," "I feel that I cannot help myself," and "I am proud of my accomplishments (reversed)." The total score is produced by summing the items, and subscale scores are produced by summing the items within the subscales. The total score has adequate internal consistency ( $\alpha=0.94)$, as do the Dysphoria $(\alpha=0.85)$, Loss of meaning and purpose $(\alpha$ $=0.87)$, Disheartenment $(\alpha=0.89)$, Helplessness $(\alpha=0.84)$, and Sense of failure $(\alpha=0.71)$ subscales (Kissane et al., 2004). The DS demonstrated good internal consistency in the present sample, with a Cronbach's alpha of 0.95 . There is support for concurrent validity of the scale and subscales, given their significant associations $(p<.01)$ in the expected directions with positive expectancies, quality of life, depression, anxiety, distress, hopelessness, and desire for death (Kissane et al., 2004; Mehnert et al., 2011; Mullane, Dooley, Tiernan, \& Bates, 2009). Despite its association with depression, divergent validity is nonetheless supported by research finding it to be a distinct construct when assessed alongside the Beck Depression Inventory II and the Patient Health Questionnaire (Fang et al., 2014; Kissane et al., 2004; Mehnert et al., 2011). For instance, anywhere from $60-85.2 \%$ of participants with moderate demoralization do not meet criteria for depression (Jacobsen et al., 2006; Mehnert et al., 2011). The scale is theorized to reflect a "mental state" (Kissane et al., 2004, p. 269) and this is supported by fluctuations in the scale in response to treatment. Specifically, scores on the scale have been reduced by as much as 66.6\% within a 24-hour period, given injection with ketamine (Zanicotti, Perez, \& Glue, 2012). Likewise, the scale exhibits significant, meaningful (e.g. Cohen's $d=0.71$; Marsa et al., 2017) reductions after brief psychotherapeutic interventions (Catanese, John, Di Battista, \& Clarke, 2009; Marsa et al., 2017). Its state-like nature and responsiveness to environment is further supported by its being predicted by joblessness, cancer diagnosis, pain, and presence and severity 
of physical problems, including in prospective investigations (De Jong, Kissane, Geessink, \& Velden, 2008; Hadnagy, Csikós, \& Nagy, 2012; Hung et al., 2010; Lee et al., 2012; Vehling et al., 2011, 2012; Vehling, Oechsle, Koch, \& Mehnert, 2013).

Multidimensional Perfectionism Scale; MPS (Appendix C; Hewitt \& Flett, 1990): The MPS is a 45-item self-report measure of perfectionism, which took approximately five to ten minutes to complete. Responses are given on a 7-point Likert scale ranging from "Disagree" to "Agree," with greater responses indicating a greater degree of endorsement of perfectionism. The measure is comprised of three subscales, reflecting self-oriented, socially prescribed, and otheroriented perfectionism. Examples of items reflecting each dimension of perfectionism are, respectively, "I strive to be the best at everything I do," "People expect nothing less than perfection from me," and "If I ask someone to do something, I expect it to be done flawlessly." A summed total score is obtained for each subscale. The scale is commonly employed in studies of perfectionism (e.g., Blankstein, Flett, Hewitt, \& Eng, 1993; Childs \& Stoeber, 2010; Klibert et al., 2005; Martin et al., 1996; Onwuegbuzie, 2000; Roxborough et al., 2012; Sherry, Hewitt, Besser, McGee, \& Flett, 2004; Stoeber, Feast, \& Hayward, 2009; Tissot \& Crowther, 2008). The scale is accessible, requiring a Grade 6-7 reading level and has been validated for use in both clinical and community samples (Hewitt \& Flett, 1991b; Hewitt, Flett, Turnbull-Donovan, \& Mikail, 1991). The self-oriented, socially prescribed, and other-oriented perfectionism subscales are reliable over time ( $r=0.88,0.75,0.85$, respectively; Hewitt \& Flett, 1991b). Moreover, the self-oriented, socially prescribed, and other-oriented perfectionism subscales appear to be internally consistent, with Cronbach's alphas of $0.86,0.87$, and 0.82 , respectively (Hewitt \& Flett, 1991b). In the present sample, the self-oriented and socially prescribed perfectionism subscales were internally reliable (Cronbach's alpha of 0.92 and 0.85 respectively). Convergent 
validity of the self-oriented perfectionism subscale is supported by its association with high standards, self-importance of performance, self-importance of goal attainment, self-criticism, and self-blame, whereas its discriminant validity is supported by a lack of association with demand for approval of others, fear of negative evaluation, authoritarianism, dominance, or otherdirected blame (Hewitt \& Flett, 1991b). Likewise, the socially prescribed perfectionism subscale evidences convergent validity by its association with demand for approval of others, fear of negative evaluation, social standards, and social importance of goal attainment; its discriminant validity is supported by a lack of relationship with high self-standards, authoritarianism, dominance, self-importance of performance, or self-importance of goal attainment (Hewitt \& Flett, 1991b).

Life Events Scale for Students; LESS (Appendix D; Clements \& Turpin, 1996; Linden, 1984): The LESS is a 36-item measure of self-reported experiencing of significant life events. It took approximately five to ten minutes to complete. Given that the LESS represents a formative measurement model, internal consistency is not assumed or expected (Bollen, 1984). The scale was developed from the Social Readjustment Rating Scale (Holmes \& Rahe, 1967) and was adapted to produce an empirically derived list of life events relevant to student and young adult populations, with item selection informed by normative data (Linden, 1984). For analyses in the present study, 25 items that have been identified by three independent raters in a prior investigation (Covault et al., 2007) to be negative, rather than neutral or ambiguous, were used. This is consistent with prior studies employing the measure to assess negative life events (Armeli, Conner, Covault, Tennen, \& Kranzler, 2008; Covault et al., 2007; Holt et al., 2013). Four of these items were adapted as follows. "Getting an unjustified low mark on a test" was adapted to read "Getting a low mark on a test," given that the original item may imply an 
appraisal component, which is a separate variable of interest in the present analyses. "Seeking psychological or psychiatric consultation" was adapted to read "Being the victim of direct or passive aggression.” The original item was not considered to be inherently negative; nonetheless, such treatment seeking may be indicative of experiences of trauma or major triggering events, lacking in the original scale. "Seriously thinking about dropping college" was adapted to read "Being placed on academic probation" as this was considered less redundant with items assessing the experience of failing courses. This was drawn from a prior measure (Sarason, 1978). Finally, "Moving out of town with parents" was likewise not considered to be inherently negative and was replaced with the similar item "Negative change in living condition of family," again from a prior measure (Sarason, 1978). Respondents indicated if they have experienced the given events or not by answering "Yes," or "No." Example items include, "Minor car accident," "Minor violation of the law," and "Major argument with girl/boyfriend." The scale is summed to produce a total score indicating number of life events experienced in the past year. The scale is commonly used in evaluations of stressful life events in young adult samples (e.g. Armeli et al., 2008; Burns, Carroll, Drayson, Whitham, \& Ring, 2003; Burns, Drayson, Ring, \& Carroll, 2002; Covault et al., 2007; Johnson, Gooding, Wood, \& Tarrier, 2010; Kranzler et al., 2012; Nikolova, Bogdan, Brigidi, \& Hariri, 2012; Phillips, Burns, Carroll, Ring, \& Drayson, 2005; Swartz, Knodt, Radtke, \& Hariri, 2015). The scale demonstrates concurrent validity, supported by its association with major personal illness, seeking psychological/psychiatric help, and failing academic courses (Linden, 1984), as well as general distress (Clements \& Turpin, 1996). Over a six month period, test-retest reliability is adequate $(r=.61$; Clements \& Turpin, 1996).

Scale for Assessing Appraisal Components (Appendix E; Smith \& Lazarus, 1993): The Scale for Assessing Appraisal Components is an eight-item nine-point Likert scale, anchored 
from 1, "Not at all," to 9, "Extremely much." The scale took approximately one minute per event to complete. From this scale, two original items were used, and one additional item was adapted. The two original items correspond to motivational relevance and self-accountability; the adapted item corresponds to motivational incongruence. Respondents rated their appraisals of a specific negative life event along these three dimensions; namely, participants rated the degree to which the event was appraised as relevant or important to the individual (motivational relevance), as falling short of standards (motivational incongruence), and as being the individual's responsibility (self-accountability). Motivational relevance is assessed by "How important was what was happening in the situation to you?" Motivational incongruence will be determined with the question: "To what extent did the results of this event fall short of your standards or expectations?" This adapted item was developed based on prior measures (e.g. Bissell \& Rask, 2010; Heidrich, 1994; Heidrich, 1999) and informed by the wording of the other two items on the scale. Self-accountability is assessed by "To what extent did you consider yourself responsible for this situation?" These three items were summed to produce a total score per event, consistent with intended use of the scale and as employed in prior investigations (Coo, Milgrom, Kuppens, \& Trinder, 2015; Lowe \& Bennett, 2003; Smith \& Lazarus, 1990; Smith et al., 1993; Smith \& Lazarus, 1993). The concurrent validity of the full eight item scale is supported by expected associations with causal attributions (Smith et al., 1993). Concurrent and discriminant validity of the three items is supported by their specific association with the emotion of guilt and the core relational theme of self-blame, relative to other appraisal dimensions, and attenuated relationships of the three items with other emotions and core relational themes (e.g. emotions of anger, fear/anxiety, and sadness; core relational themes of other-blame, danger/threat, and irrevocable loss; Smith et al., 1993; Smith \& Lazarus, 1993). For 
instance, appraisals of events as important (i.e. relevant), as incongruent with desired outcomes, and as one's own responsibility are theorized to be related to guilt through a pathway of selfblame, whereas appraisals of an event as important, as incongruent with desired outcomes, and as externally caused are theorized to be related to anger through a pathway of other-blame; the scale exhibits these specific associations (Bennett, Lowe, \& Honey, 2003; Smith et al., 1993; Smith \& Lazarus, 1993). Furthermore, the scale demonstrates high test-retest reliability (Bennett et al., 2003), with no significant effect of time on these three appraisals across a one-week interval. The scale demonstrated poor internal consistency in the present sample, with an average Cronbach's alpha across the negative life events of 0.56 (range $=-0.20$ to 0.75 ). This may be an artifact of the few number of items on the measure (Bernstein \& Nunnally, 1994; Streiner, 2003; Tavakol \& Dennick, 2011). However, it is also conceivable that motivational relevance, motivational incongruence, and self-accountability represent distinct constructs which would not necessarily be expected to change together.

\section{Procedure}

Participants were recruited through the University of South Florida psychology department's SONA pool. Before beginning the study, participants provided IRB-approved informed consent using an online form. This online informed consent document provided participants information about study involvement, such as potential risks, potential benefits, background, purpose of study, study procedure, participant rights, and confidentiality policies. Participants who provided informed consent then began the study. The study took approximately 30 minutes to complete in total. Participants received one extra credit point for each half hour of participation. Participants were allowed to end the study at any time without penalty. After completion of the study, participants were debriefed. The online debriefing form included further 
detail on the purpose of the study, contact information for the study investigator, and a list of campus and community-based mental health resources that the participant may utilize. All participant data has been de-identified; identifying information was replaced with an anonymous participant number. All study data is stored on a secured, password protected server and is accessible only to authorized study personnel.

\section{Proposed Data Analysis}

Power Analysis. Sample size was determined with consideration to calculations of statistical power using G*POWER 3.1.9.2. Power analysis was based on the ability to detect the additional variance accounted for by the proposed two-way interaction term. The power analysis indicated that a sample size of 199 would be needed to detect a small to medium effect $\left(\Delta R^{2}=\right.$ $.04)$ with power $=.80$ and $\alpha=.05$.

Preliminary Analyses. Total scores were produced for the study variables. Items from the MPS, LESS, Scale for Assessing Appraisal Components, and DS measures were summed to produce a total score and respective subscale scores as relevant to the measure. Higher scores indicated greater perfectionism; a greater number of past-year negative life events; greater appraisal of events as relevant, falling short of standards, and internally caused; and greater demoralization, respectively. Descriptive statistics were run on demographic and study variables, to characterize the sample and to review means, standard deviations, and ranges of scores. The skewness and kurtosis of study variables were assessed to examine the distribution of the variables. Variable distributions were assessed for normality and outliers. Pearson correlation coefficients were produced to examine the relationship of study variables, with particular attention to the relationship of the MPS, LESS, and the Scale for Assessing Appraisal 
Components score to the DS. Internal consistency reliability in the present sample was assessed as relevant with Cronbach's alpha.

Regression and Moderation Analyses. To examine whether greater levels of perfectionism relate to greater demoralization (Hypothesis 1A, 1B), a standard multiple regression analysis was used. Self-oriented and socially prescribed perfectionism were entered as predictors of demoralization. The beta weights were examined to determine if both contribute unique, statistically significant variance to the criterion. The magnitude of the effects was compared (Hypothesis 1C) using the Hotelling-Williams test for dependent samples (Steiger, 1980). To examine the effect of negative life events on demoralization (Hypothesis 2A), a linear regression was run with negative life events as the predictor and demoralization as the outcome. To evaluate the degree to which the experience of negative life events moderates the relationship between perfectionism and demoralization (Hypothesis 2B, 2C), hierarchical multiple regression analyses were run, consistent with recommended procedures (Baron \& Kenny, 1986; Holmbeck, 1997). Prior to analyzing interactive effects, the predictor and moderator variables were meancentered (Aiken, West, \& Reno, 1991). In each analysis, demoralization was the dependent variable and negative life events was the moderator. Perfectionism (i.e. self-oriented or socially prescribed) was the predictor variable. Perfectionism and the proposed moderator of negative life events were entered in the first step of the equation. The interaction term, representing the interaction between perfectionism and negative life events, was entered in the second step. Evidence for an interactive effect was determined by examining the significance of the interaction effect and determining if the new model explained significantly more variance than the model without the interaction effect. Similarly, to evaluate the relationship between perfectionism and appraisal (Hypothesis 3Ai, 3Bi) and the degree to which the experience of 
negative life events moderates the relationship between perfectionism and appraisal (Hypothesis 3Aii, 3Bii), hierarchical multiple regression analyses were run. In each analysis, appraisal was the dependent variable and negative life events was the moderator. Perfectionism (i.e. selforiented or socially prescribed) was the predictor variable. Perfectionism and the proposed moderator of negative life events were entered in the first step of the equation. The interaction term, representing the interaction between perfectionism and negative life events, was entered in the second step. Evidence for an interactive effect was determined by examining the significance of the interaction effect and determining if the new model explained significantly more variance than the model without the interaction effect.

To evaluate the degree to which appraisal mediated the effects of perfectionism and negative life events on demoralization (Hypothesis 3A, 3B), mediation analyses were conducted using bootstrapped samples, as recommended by Preacher and Hayes (2004). Regression analyses were first conducted examining pathways $a, b$, and $c$ (see Figure 2) to inform plausibility of mediation. The Preacher and Hayes (2004) bootstrap method was used, corresponding to Model 8 in the PROCESS Macro (Hayes, 2013), to generate point estimates and confidence intervals of the mediation effect at average, below average, and above average experiencing of negative life events. Mediation was considered tenable if zero did not fall within the bounds of the confidence intervals. 


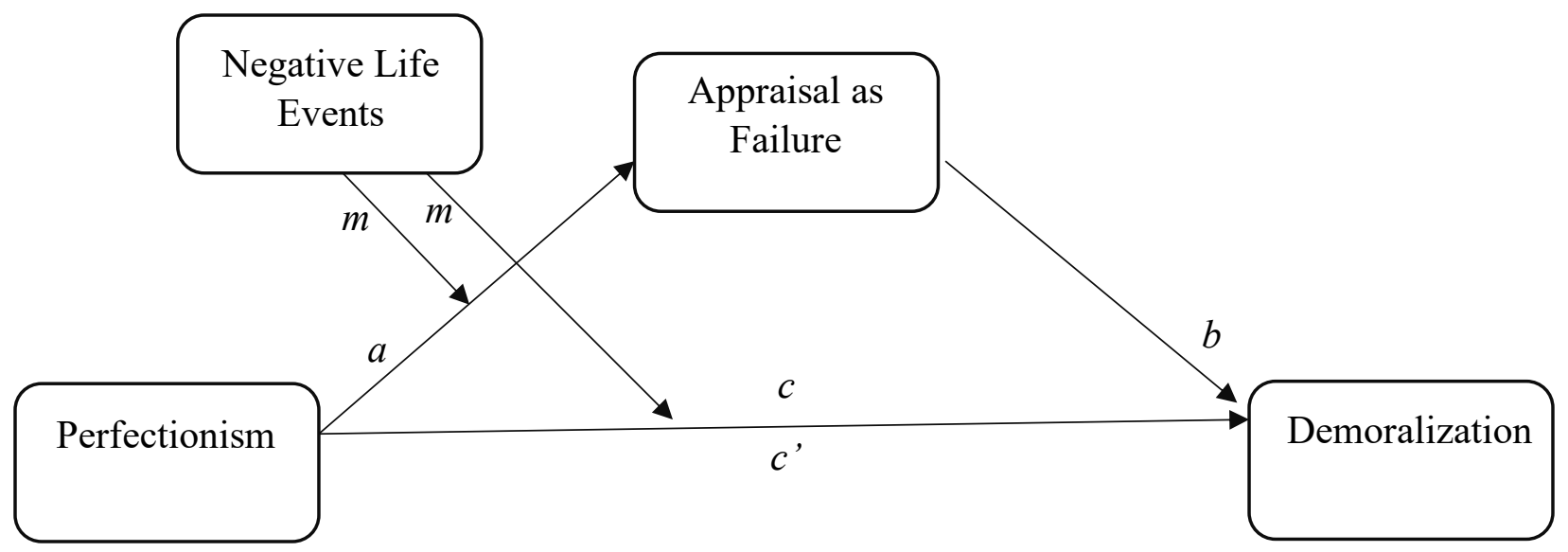

Figure 2. Statistical model of the mediated effect of perfectionism and negative life events on demoralization through appraisal. 


\section{Results}

\section{Data Screening}

Careless responding was assessed by checking for accurate completion of the five included attention checks. Of the 364 participants, 44 were removed from final analyses for failing at least one of the attention checks. Chi-square tests of independence were performed to test for demographic predictors of careless responding. Participants removed for careless responding were more likely to be male $(\chi 2=6.50, \mathrm{p}=.04)$ and to report never having been diagnosed with a mental health disorder $(\chi 2=4.09, \mathrm{p}=.04)$. There were no other demographic differences in the final sample.

Descriptive statistics were run on those participants who passed attention checks $(n=$ $320)$ and had relatively complete data $(n=320)$. Scatterplots of the study variables were produced to visually assess for linearity of relationships. Linear relationships amongst study variables were supported. Data was screened for nonnormality; if skewness and kurtosis were between positive or negative two, scores were considered normally distributed (Cameron, 2004). No study variables were nonnormal; skewness and kurtosis are noted in descriptives sections. Data was further screened for nonnormality by examining the probability-probability plot (P-P Plot). Normality of study variables was supported. Data was screened for multivariate outliers by producing Mahalanobis distance values. With a cutoff of $\chi^{2}(\mathrm{df}), \alpha=.001$, where $\mathrm{df}=$ the number of independent variables, one multivariate outlier was identified for negative life events and demoralization $(\mathrm{MD}=24.96)$, one multivariate outlier was identified for appraisals and demoralization $(\mathrm{MD}=17.59)$, and one multivariate outlier was identified for negative life events 
and appraisals $(\mathrm{MD}=25.99)$. Analyses were run with and without participants with outliers $(n=$ 2) and did not differ. Thus, analyses are reported with these outliers included. Data was screened for heteroscedasticity by examining the distribution of residual values plotted against predicted values. Homoscedasticity of study variables was supported. Data was screened for multicollinearity by examining the variance inflation factor (VIF). The study variables were not multicollinear (maximum VIF = 1.05; Hair et al., 2010). Missing items are noted in descriptives sections as applicable.

\section{Descriptive Statistics}

Multidimensional Perfectionism Scale (MPS). Means and standard deviations for the Socially Prescribed Perfectionism (SPP) and Self-Oriented Perfectionism (SOP) subscales of the MPS are presented in Table 2. No participants had missing data on this measure. Responses on the subscales were normally distributed, with skewness and kurtosis within a range of positive and negative one. Scores obtained in the present sample were not significantly different from those obtained in other samples reported elsewhere for both the SPP $\left(M c^{1}=56.22 ; t(409)=\right.$ $1.25, p=0.21$; Hewitt, Flett, \& Weber, 1994) and SOP $(M c=72.58 ; t(409)=-0.09, p=0.93$; Hewitt, Flett, \& Weber, 1994) subscales.

Life Events Scale for Students (LESS). The mean and standard deviation of the LESS are presented in Table 2. No participants had missing data on this measure. The fourth negative life event ("Getting a low mark on a test;" $N=236,73.80 \%$ ) was the most frequently endorsed past-year event, and the seventh negative life event ("Getting kicked out of college;" $N=0$, $0.00 \%$ ) was the least frequently endorsed. Responses on the scale were normally distributed,

\footnotetext{
${ }^{1}$ Means from compared studies
} 
with skewness and kurtosis within a range of positive and negative two. Scores obtained in the present sample were not significantly different from those obtained in other samples reported elsewhere $(M c=4.28 ; t(1162)=0.30, p=0.76$; Holt et al., 2013).

Scale for Assessing Appraisal Components. The mean and standard deviation for the Scale for Assessing Appraisal Components are presented in Table 2. Twelve participants reported experiencing none of the assessed negative life events in the past year and thus were ineligible to supply ratings of appraisals of these past-year negative life events. Ten life events had missing data on their corresponding appraisal components. That is, ten life events had at least one participant who reported experiencing the event but failed to provide an appraisal response. Given that PROCESS is unable to accommodate the multiple imputation procedure in SPSS (Hayes, 2019), mean imputation was used to estimate the missing values prior to analyses; the mean of the respective appraisal component for the respective event (e.g. mean motivational relevance for event two) replaced the missing value. One participant who had experienced the second negative life event ("Major personal injury or illness") had missing values on all three appraisal components $(1.89 \%$ missing data). Twenty-three participants who had experienced the third negative life event ("Major argument with parents") had missing data across 29 values (7.38\% missing data). One participant who had experienced the fourth negative life event (“Getting a low mark on a test") had missing values on all three appraisal components $(0.42 \%$ missing data). One participant who had experienced the sixth negative life event ("Minor violation of the law 'e.g. speeding ticket') had missing values on all three appraisal components (2.86\% missing data). One participant who had experienced the eighth negative life event ("Being the victim of direct or passive aggression") had missing values on all three appraisal components (1.15\% missing data). One participant who had experienced the ninth negative life 
event ("Minor car accident") had missing values on all three appraisal components ( $1.32 \%$ missing data). One participant who had experienced the $12^{\text {th }}$ negative life event ("Negative change in living condition of family") had missing values on two appraisal components (1.67\% missing data). One participant who had experienced the $15^{\text {th }}$ negative life event ("Major change of health in close family member") had missing values on all three appraisal components $(1.01 \%$ missing data). One participant who had experienced the $16^{\text {th }}$ negative life event ("Major car accident [car wrecked, people injured]") had a missing value on one appraisal component $(1.52 \%$ missing data). Two participants who had experienced the $21^{\text {st }}$ negative life event ("Major argument with boy/girlfriend") had missing data across four values (1.63\% missing data). Responses on the scale were normally distributed, with skewness and kurtosis within a range of positive and negative two. The scale was changed from an 11-point to a 9-point Likert range at the author's advice (C. A. Smith, personal communication, August 22, 2018). Compared to two identified studies which employed the 9-point Likert scale, scores in the present sample were significantly higher $[(M c=16.60 ; t(425)=5.99, p<0.001$; Lowe \& Bennett, 2003), $(M c=14.60$; $t(466)=12.07, p<0.001$; Lowe et al., 2003)]. This appears to reflect greater self-accountability, which may be expected given that one study (Lowe et al., 2003) was examining appraisals of women who had been referred to breast cancer diagnosis clinics; that is, individuals may be less likely to feel responsible for a breast cancer diagnosis than for the negative life events assessed in the present study. The other study (Lowe \& Bennet, 2003) asked a sample of nurses to write of their most stressful work incident in the previous month. Nurses may likewise be less likely to feel accountable for these stressors relative to the negative life events of the present study. This study also had a notably lower motivational incongruence score, which may be related to the adapted nature of this item in the present study. Qualitatively, studies employing the 11-point 
scale demonstrated approximately comparable scores [e.g. mean in present sample $=19.52$ (72.30\% of maximum value); $M c=25.03$ (75.85\% of maximum value; Smith \& Lazarus, 1993$)$ ].

Demoralization Scale (DS). The mean and standard deviation for the DS are presented in Table 2. No participants had missing data on this measure. Responses on the scale were normally distributed, with skewness and kurtosis within a range of positive and negative one. While there are no studies assessing the prevalence of demoralization — as reflected by the DS — in non-medical populations (Tecuta, Tomba, Grandi, \& Fava, 2015), scores obtained in the present sample were significantly greater $(M c=19.94 ; t(418)=2.59, p=0.01$; Mullane et al., 2009) and lower $(M c=30.82 ; t(418)=-2.80, p<0.01$; Kissane et al., 2004) than those obtained in samples of patients with advanced cancer. While the higher scores obtained by Kissane et al. (2004) are expected given the chosen sample and comorbid depressive symptomatology (e.g. $32 \%$ of sample endorsed moderate to severe symptomatology), the lower scores obtained by Mullane et al. (2009) may be due to protective demographic characteristics (e.g. $83 \%$ of sample was Roman Catholic, $51 \%$ of sample was married) and lower rates of depressive symptomatology (e.g. $21 \%$ of sample endorsed moderate to severe symptomatology). 
Table 2. Descriptive Statistics

\begin{tabular}{|c|c|c|c|c|c|c|c|c|}
\hline$\underline{\text { Variable }}$ & $\underline{\mathrm{N}}$ & $\underline{\text { Mean }}$ & $\underline{\mathrm{SD}}$ & $\underline{\text { Min }}$ & $\underline{\operatorname{Max}}$ & $\underline{\text { Skewness }}$ & $\underline{\text { Kurtosis }}$ & $\frac{\text { Cronbach's }}{\underline{\text { Alpha }}}$ \\
\hline \multicolumn{9}{|l|}{$\begin{array}{l}\text { Multidimensional } \\
\text { Perfectionism Scale }\end{array}$} \\
\hline \multicolumn{9}{|l|}{ Socially Prescribed } \\
\hline $\begin{array}{l}\text { Self-Oriented } \\
\text { Perfectionism }\end{array}$ & 320 & 72.77 & 16.85 & 26.00 & 105.00 & -0.02 & -0.60 & 0.92 \\
\hline $\begin{array}{l}\text { Life Events Scale for } \\
\underline{\text { Students }}\end{array}$ & 320 & 4.34 & 2.74 & 0.00 & 18 & 0.98 & 1.66 & NA \\
\hline $\begin{array}{l}\text { Scale for Assessing } \\
\text { Appraisal Components }\end{array}$ & $308^{\mathrm{a}}$ & 19.54 & 3.50 & 4.85 & 27.00 & -0.45 & 1.15 & $0.56^{\mathrm{b}}$ \\
\hline Demoralization Scale & 320 & 25.06 & 18.01 & 0.00 & 87.00 & 0.97 & 0.70 & 0.95 \\
\hline
\end{tabular}




\section{Correlations}

Prior to testing study hypotheses, Pearson correlation coefficients were produced to examine the relationships of study variables. Study correlations are presented in Table 3 . As predicted, socially prescribed perfectionism was related to appraisals $(r=.22, p<.01)$ and to demoralization $(r=.36, p<.01)$. Self-oriented perfectionism was likewise related to appraisals $(r=.19, p<.01)$ and to demoralization $(r=.13, p<.05)$. Appraisals in turn related to demoralization $(r=.15, p<.01)$.

Table 3. Intercorrelations of Study Variables

\begin{tabular}{llllll}
\hline & 1 & 2 & 3 & 4 & 5 \\
\hline 1. SPP & 1 & & & & \\
2. SOP & $.51^{* *}$ & 1 & & & \\
3. NLE & .04 & .02 & 1 & & \\
4. Appraisal & $.22^{* *}$ & $.19^{* *}$ & -.00 & 1 & \\
5. Demoralization & $.36^{* *}$ & $.13^{*}$ & $.20^{* *}$ & $.15^{* *}$ & 1 \\
\hline
\end{tabular}

$* p<.05, * * p<.01$, All correlations are Pearson's $\mathrm{r}$.

Note the sample for this correlation table is $N=320$, except where noted. $a-N=308$.

\section{Relationship between Study Variables and Relevant Covariates}

To assess for potential confounding effects of demographic variables on the variables of interest, Pearson correlations and one-way ANOVAs were run with demographic data predicting study variables. For categorical demographic variables, cells with fewer than two participants (e.g. participants identifying as asexual, $n=1$ ) were excluded. Age was negatively correlated with appraisal as personal failure $(r=-0.12, p<.05)$. Race/ethnicity was significantly related to demoralization $F(10,301)=1.98, p<.05$. Post-hoc analyses with Bonferroni corrections indicated that participants identifying as Asian American reported greater demoralization than participants identifying as Hispanic/Latinx $(\bar{D}=15.92, p=.008)$. There were no other significant 
differences in demoralization by race/ethnicity. Sexual orientation was significantly related to demoralization $F(3,315)=7.09, p<.001$ and negative life events $F(3,315)=3.94, p<.01$. Post-hoc analyses with Bonferroni corrections indicated that individuals who identified as bisexual $(\bar{D}=11.24, p=.003)$ and other sexuality $(\bar{D}=17.65, p=.03)$ reported higher demoralization than participants who identified as heterosexual. Participants identifying as bisexual likewise reported experiencing significantly more negative life events $(\bar{D}=1.29, p=$ $0.05)$ than participants identifying as heterosexual. There were no other significant relationships among demographic items and study variables. Accordingly, analyses were conducted with and without including age, race/ethnicity, and sexual orientation as covariates. Results did not change and are reported without controlling for these demographics.

\section{Hypothesis Testing}

Hypothesis 1A: It was hypothesized that socially prescribed perfectionism would contribute unique, statistically significant variance to demoralization within the past two weeks. Multiple regression analyses were used to test this hypothesis. Socially prescribed and selforiented perfectionism were entered as predictors of demoralization in a standard multiple regression. Together, the predictors accounted for $14 \%$ of the variance in demoralization $\left(R^{2}=\right.$ $.14, F(2,317)=25.11, p<.001)$. Next the semi-partial correlations of the predictors were examined for significance and magnitude. Results from the multiple regression analysis supported this hypothesis. Higher socially prescribed perfectionism related uniquely to greater recent demoralization $(s r=0.35, p<.001)$. A hierarchical regression was run to assess the additional variance contributed to demoralization by socially prescribed perfectionism, after accounting for the variance contributed by self-oriented perfectionism. In the first step, selforiented perfectionism was entered as a predictor of demoralization; socially prescribed 
perfectionism was entered in the second step. After accounting for variance contributed by selforiented perfectionism, socially prescribed perfectionism as hypothesized accounted for an additional $12.00 \%$ of variance in demoralization, a moderate effect $\left(r=0.35, \Delta R^{2}=.12, F(1\right.$, $317)=44.40, p<.001)$.

Hypothesis 1B: It was hypothesized that self-oriented perfectionism would contribute unique, statistically significant variance to demoralization within the past two weeks. Results from the multiple regression analysis did not support this hypothesis. Higher self-oriented perfectionism was not uniquely significantly related to greater recent demoralization $(s r=-0.07$, $p=.18)$.

Hypothesis 1C: It was hypothesized that the unique main effect of socially prescribed perfectionism on feelings of demoralization would be significantly greater than the unique main effect of self-oriented perfectionism on feelings of demoralization. Results from a HotellingWilliams test for dependent samples (Lee \& Preacher, 2013; Steiger, 1980) supported this hypothesis; the effect of socially prescribed perfectionism on demoralization was significantly greater than the effect of self-oriented perfectionism on demoralization $(z=4.503, p<.0001)$, corresponding to a medium effect size (Cohen's $d=0.52$; Lenhard \& Lenhard, 2016).

Hypothesis 2A: It was hypothesized that as individuals experienced an increasing number of negative life events, they would experience increasingly greater feelings of demoralization as a main effect. Results from a simple linear regression supported this hypothesis; experiencing a greater number of past-year negative life events related to reports of greater recent demoralization $(B=1.31, p<.001)$. Negative life events explained $3.90 \%$ of the variance in demoralization, a small to moderate effect $\left(r=0.20, R^{2}=.04, F(1,318)=13.07, p<\right.$ $.001)$. 
Hypothesis 2B: It was hypothesized that socially prescribed perfectionism would increasingly relate to demoralization as individuals experienced a greater number of negative life events. A hierarchical linear regression was used to test this hypothesis. All assumptions for hierarchical linear regression and moderation were met. Prior to testing moderation, the predictors, socially prescribed perfectionism and negative life events, were mean-centered (Aiken, West, \& Reno, 1991). Demoralization was entered as the criterion. In the first step, mean-centered socially prescribed perfectionism and negative life events were entered as main effects. The interaction term, representing the interaction between socially prescribed perfectionism and negative life events, was entered in the second step. The first step accounted for statistically significant variance in demoralization $\left(R^{2}=.17, F(2,317)=31.44, p<.001\right)$, corresponding to a small to moderate effect size $(r=0.41)$. Socially prescribed perfectionism $(B$ $=0.46, p<.001)$ and negative life events $(B=1.21, p<.001)$ predicted greater demoralization. The interaction term was not significant $(B=0.02, p=.46)$, and the new model did not contribute significantly more variance than the model without the interaction term $\left(\Delta R^{2}=.00\right.$, $F(1,316)=0.54, p=.46)$. Accordingly, the results of the hierarchical linear regression did not support this hypothesis.

Hypothesis 2C: It was hypothesized that self-oriented perfectionism would increasingly relate to demoralization as individuals experienced a greater number of negative life events. A hierarchical linear regression was used to test this hypothesis. All assumptions for hierarchical linear regression and moderation were met. Prior to testing moderation, the predictors, selforiented perfectionism and negative life events, were mean-centered (Aiken, West, \& Reno, 1991). Demoralization was entered as the criterion. In the first step, mean-centered self-oriented perfectionism and negative life events were entered as main effects. The interaction term, 
representing the interaction between self-oriented perfectionism and negative life events, was entered in the second step. The first step accounted for statistically significant variance in demoralization $\left(R^{2}=.05, F(2,317)=9.12, p<.001\right)$, corresponding to a small to moderate effect size $(r=0.22)$. Self-oriented perfectionism $(B=0.13, p<.05)$ and negative life events $(B=1.29$, $p<.001)$ predicted greater demoralization. The interaction term was not significant $(B=0.01, p$ $=.69$ ), and the new model did not contribute significantly more variance than the model without the interaction term $\left(\Delta R^{2}=.00, F(1,316)=0.16, p=.69\right)$. Accordingly, the results of the hierarchical linear regression did not support this hypothesis.

Hypothesis 3A: It was hypothesized that the interactive effect of socially prescribed perfectionism and negative life events on demoralization would operate in part through a pathway of appraisal. Specifically, it was hypothesized that, in the context of negative life events, individuals who had increasingly elevated socially prescribed perfectionism would increasingly appraise these negative life events as personal failures and would accordingly feel increasingly demoralized. Because the proposed interaction between socially prescribed perfectionism and negative life events in predicting demoralization was not supported (Hypothesis 2B), this hypothesis likewise was not supported. However, Hypotheses 3Ai, 3Aii, and 3Aiii/3Biii were tested (see Figure 3). To examine the effect of appraisals on demoralization (Hypothesis 3Aiii/3Biii), a simple linear regression was run, with appraisal entered as the predictor and demoralization entered as the criterion. Results from this simple linear regression supported this hypothesis; greater appraisal as failure related to reports of greater recent demoralization $(B=0.79, p<.01)$. Appraisal explained $2.40 \%$ of the variance in demoralization, a small effect $\left(r=0.15, R^{2}=.02, F(1,306)=7.38, p<.01\right)$. To examine the interactive effect of socially prescribed perfectionism and negative life events on appraisals (Hypothesis 3Aii), a 
hierarchical linear regression was run. All assumptions for hierarchical linear regression and moderation were met. Prior to testing moderation, the predictors, socially prescribed perfectionism and negative life events, were mean-centered (Aiken, West, \& Reno, 1991). Appraisals were entered as the criterion. In the first step, mean-centered socially prescribed perfectionism and negative life events were entered as main effects. The interaction term, representing the interaction between socially prescribed perfectionism and negative life events, was entered in the second step. The first step accounted for statistically significant variance in appraisals, a small to moderate effect $\left(r=0.22, R^{2}=.05, F(2,305)=7.79, p=.001\right)$. Socially prescribed perfectionism $(B=0.06, p<.001)$ predicted greater appraisal as failure, supporting Hypothesis $3 \mathrm{Ai}$; socially prescribed perfectionism explained $4.50 \%$ of the variance in appraisals, a small to moderate effect $\left(r=0.22, R^{2}=0.05\right)$. Negative life events did not significantly relate to appraisals $(B=-0.01, p=.88)$. The interaction term was not significant $(B=0.00, p=.57)$, and the new model did not contribute significantly more variance than the model without the interaction term $\left(\Delta R^{2}=.00, F(1,304)=0.32, p=.57\right)$. Accordingly, the results of the hierarchical linear regression did not support an interaction between socially prescribed perfectionism and negative life events in predicting appraisals (Hypothesis 3Aii). 


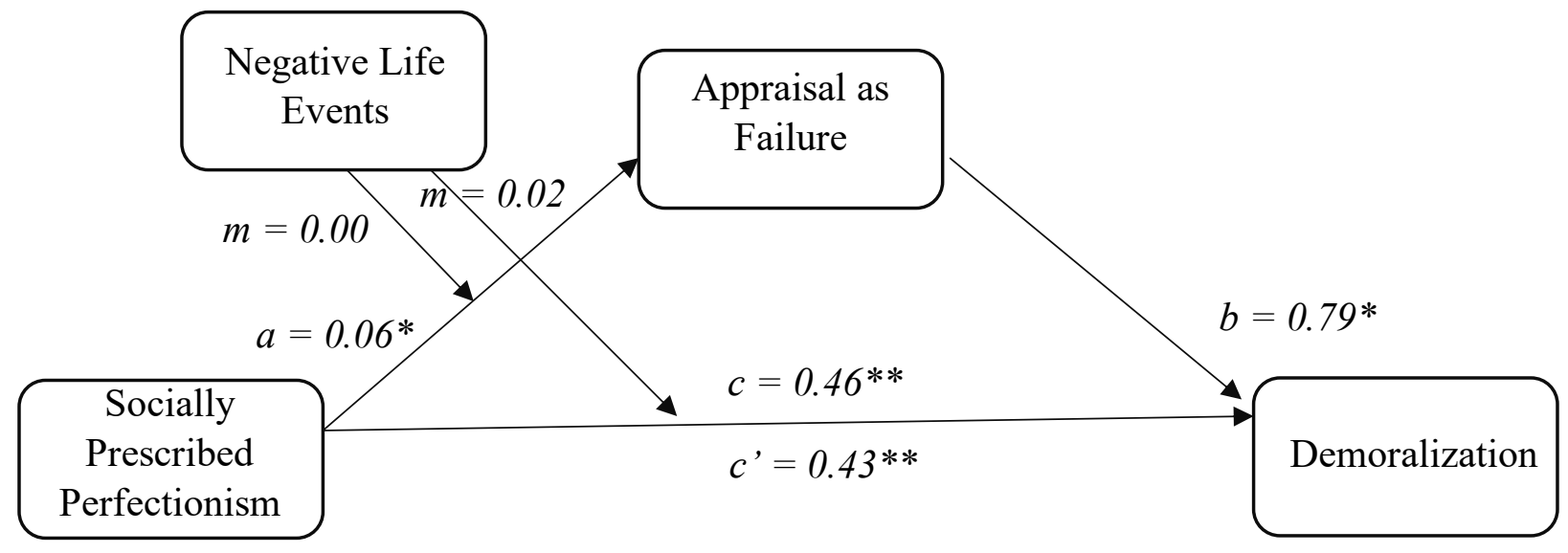

Figure 3. Statistical model of the proposed effect of socially prescribed perfectionism and negative life events on demoralization through appraisal. * = significant at $p<.05, * *=$ significant at $p<.001$.

Hypothesis 3B: It was hypothesized that the interactive effect of self-oriented perfectionism and negative life events on demoralization would operate in part through a pathway of appraisal. Specifically, it was hypothesized that, in the context of negative life events, individuals who had increasingly elevated self-oriented perfectionism would increasingly appraise these negative life events as personal failures and would accordingly feel increasingly demoralized. Because the proposed interaction between self-oriented perfectionism and negative life events in predicting demoralization was not supported (Hypothesis 2C), this hypothesis likewise was not supported. Hypotheses 3Bi and 3Bii were tested (see Figure 4). To examine the interactive effect of self-oriented perfectionism and negative life events on appraisals, a hierarchical linear regression was run. All assumptions for hierarchical linear regression and moderation were met. Prior to testing moderation, the predictors, self-oriented perfectionism and negative life events, were mean-centered (Aiken, West, \& Reno, 1991). Appraisals were entered as the criterion. In the first step, mean-centered self-oriented perfectionism and negative life events were entered as main effects. The interaction term, representing the interaction between 
self-oriented perfectionism and negative life events, was entered in the second step. The first step accounted for statistically significant variance in appraisals, a small to moderate effect $(r=0.20$, $\left.R^{2}=.04, F(2,305)=5.78, p=.003\right)$. Self-oriented perfectionism $(B=0.04, p=.001)$ predicted greater appraisal as failure, supporting Hypothesis 3Bi; self-oriented perfectionism explained $3.70 \%$ of the variance in appraisals, a small to moderate effect $(r=0.19)$. Negative life events did not significantly relate to appraisals $(B=0.00, p=1.00)$. The interaction term was not significant $(B=0.01, p=.09)$, and the new model did not contribute significantly more variance than the model without the interaction term $\left(\Delta R^{2}=.01, F(1,304)=2.98, p=.09\right)$. Accordingly, the results of the hierarchical linear regression did not support an interaction between selforiented perfectionism and negative life events in predicting appraisals (Hypothesis 3Bii).

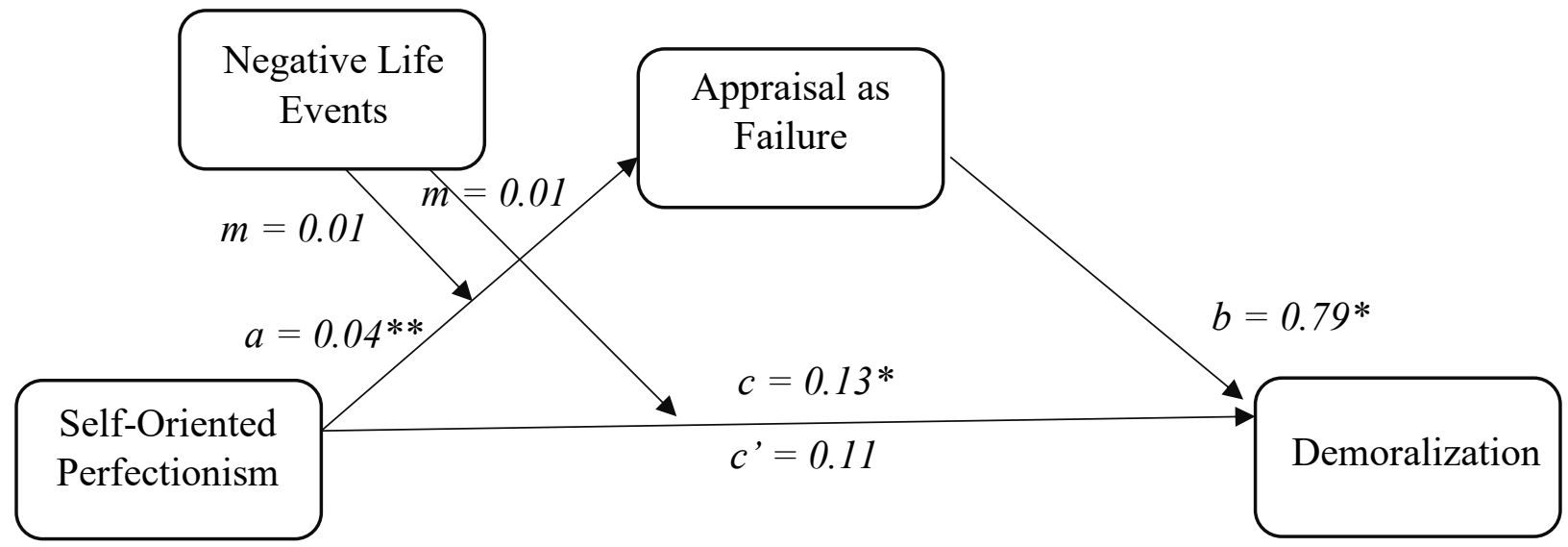

Figure 4. Statistical model of the proposed effect of self-oriented perfectionism and negative life events on demoralization through appraisal. ${ }^{*}=p<.05,{ }^{* *}=p<.01$.

\section{Supplemental Analyses}

\section{Main effect of socially prescribed perfectionism on demoralization through}

appraisal. There was not support for an interaction between socially prescribed perfectionism and negative life events in predicting demoralization through appraisal. However, there appeared 
to be support for a main effect of socially prescribed perfectionism on demoralization through appraisal, given the significant $a, b$, and $c$ pathways (see Figure 3). To test this supplemental hypothesis, mediation analyses were conducted with 10,000 bootstrapped samples (Preacher and Hayes, 2004). Model 4 in the PROCESS Macro (Hayes, 2013) was run to generate the point estimate and confidence interval of the mediation effect. Mediation was considered tenable if zero did not fall within the bounds of the confidence interval. Results of these analyses indicated that the indirect main effect of socially prescribed perfectionism on demoralization through appraisal was not significant $(a b=0.0221,95 \% \mathrm{CI}[-0.0034,0.0619])$.

\section{Main effect of self-oriented perfectionism on demoralization through appraisal.}

There was not support for an interaction between self-oriented perfectionism and negative life events in predicting demoralization through appraisal. However, there appeared to be support for a main effect of self-oriented perfectionism on demoralization through appraisal, given the significant $a, b$, and $c$ pathways (see Figure 4). To test this supplemental hypothesis, mediation analyses were conducted with 10,000 bootstrapped samples (Preacher and Hayes, 2004). Model 4 in the PROCESS Macro (Hayes, 2013) was run to generate the point estimate and confidence interval of the mediation effect. Mediation was considered tenable if zero did not fall within the bounds of the confidence interval. Results of these analyses indicated that the indirect main effect of self-oriented perfectionism on demoralization through appraisal was significant, but accounted for very little variance in the criterion $\left(a b=0.0275,95 \%\right.$ CI $[0.0049,0.0702], R^{2}=$ 0.006). However, this small $\mathrm{R}^{2}$ is somewhat consistent with results from Fairchild et al. (2009) whose statistical simulations of this measure of effect size for mediation produced $R^{2}$ values ranging from .001 to .280 . The authors write, "Small values of the $\mathrm{R}^{2}$ med occur because the measure isolates the variance explained in a dependent measure by squaring various elements 
involved in the product of two variables. Squaring or taking the product of any estimate reduces its size if values are less than one" (Fairchild et al., 2009, p. 493). That is, the effect size of the mediation is produced from squared partial correlations, and partial correlations are necessarily less than one; squaring a value of less than one produces a smaller value. Accordingly, the $\mathrm{R}^{2}$ effect size for mediation will be constrained by the squaring of the $a, b$, and c' pathways.

Self-oriented perfectionism predicted greater appraisal as failure ( $a$ path; $B=0.04, p<$ .001 ), which in turn predicted greater demoralization ( $b$ path; $B=0.69, p<.05)$, and the direct pathway from self-oriented perfectionism to demoralization was no longer significant after accounting for the mediator $\left(c^{\prime}=0.1080,95 \%\right.$ CI $\left.[-0.0129,0.2290]\right)$, supporting full mediation. Together, the variables accounted for $3.33 \%$ of the variance in demoralization $\left(R^{2}=.03\right)$. These results are presented in Figure 5.

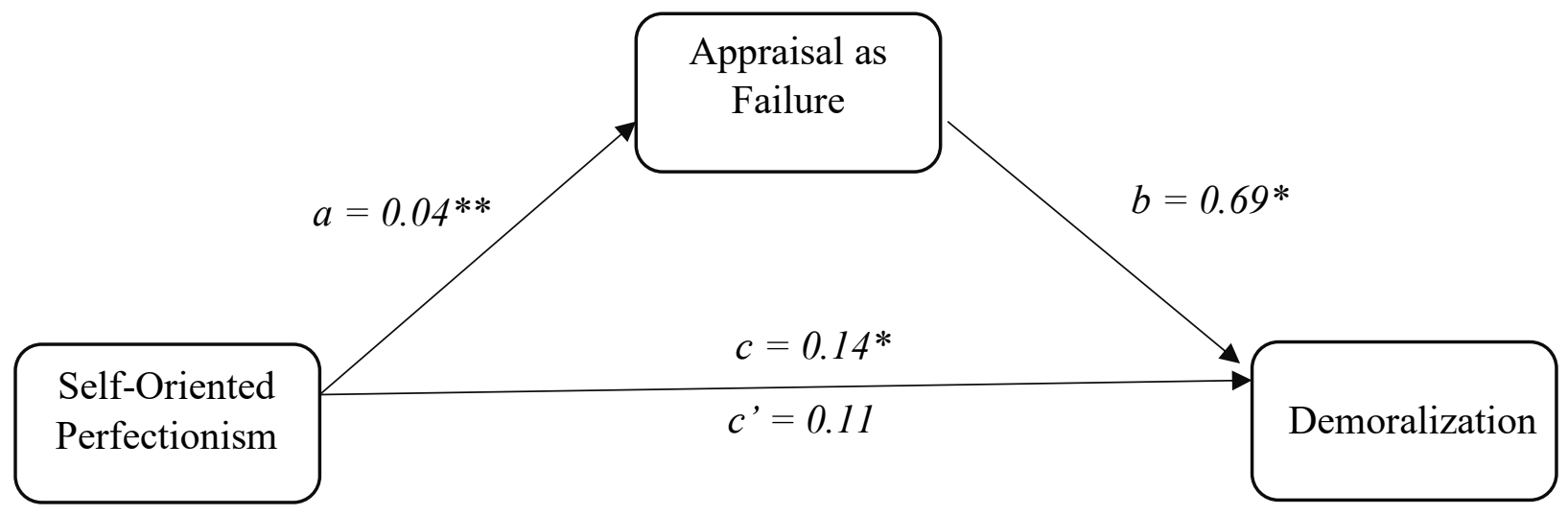

Figure 5. Statistical model of the proposed effect of self-oriented perfectionism on demoralization through appraisal. $*=p<.05, * *=p<.001$.

Examining the effect of socially prescribed perfectionism. To examine the unique effect of self-oriented perfectionism on demoralization through appraisal, the model was rerun with socially prescribed perfectionism included as a covariate. After controlling for socially 
prescribed perfectionism, the indirect effect of self-oriented perfectionism on demoralization through appraisal was not significant ( $a b=0.0097,95 \%$ CI $[-0.0025,0.0430])$. The effect of self-oriented perfectionism on appraisal was no longer significant ( $a$ path; $B=0.02, p=.10$ ), nor was the effect of appraisal on demoralization ( $b$ path; $B=0.44, p=.12$ ).

\section{Main effect of self-oriented perfectionism on demoralization through socially}

prescribed perfectionism. Given the effect of including socially prescribed perfectionism in the model, an exploratory serial mediation model was run. Self-oriented perfectionism was entered as the independent variable, socially prescribed perfectionism was entered as the first mediator, appraisal as failure was entered as the second mediator, and demoralization was entered as the criterion. The total indirect effect considers the role of all included mediating variables; examination of the $95 \%$ confidence interval of the total estimated indirect effect supported mediation $\left(a b=0.2300,95 \%\right.$ CI $\left.[0.1497,0.3297], R^{2}=0.02\right)$. Accordingly, specific indirect pathways were examined. After accounting for the effect of socially prescribed perfectionism on appraisal, self-oriented perfectionism no longer significantly predicted appraisal ( $a 2$ path; $B=$ $0.02, p=.10)$. Examination of the $95 \%$ confidence interval of the indirect effect of self-oriented perfectionism on appraisal through socially prescribed perfectionism supported mediation $($ ald $=$ $0.2225,95 \%$ CI $\left.[0.1477,0.3159], R^{2}=0.01\right)$. Likewise, after accounting for the effect of socially prescribed perfectionism on demoralization, self-oriented perfectionism no longer significantly predicted demoralization ( $c^{\prime}$ path; $B=-0.09, p=.17$ ). Examination of the $95 \%$ confidence interval of the indirect effect of self-oriented perfectionism on demoralization through socially prescribed perfectionism supported mediation $\left(a 1 b 1=0.2125,95 \% \mathrm{CI}[0.1355,0.3075], R^{2}=\right.$ 0.01). As reviewed, the direct effect of self-oriented perfectionism on appraisal was nonsignificant, as was the effect of appraisal on demoralization; consistent with this, the $95 \%$ 
confidence interval of the indirect pathway from self-oriented perfectionism to demoralization through appraisal did not support mediation $(a 2 b 2=0.0097,95 \%$ CI $[-0.0025,0.0430])$. When both mediators were included, the effect of self-oriented perfectionism on demoralization was no longer significant ( $c$ ' path; $B=-0.09, p=.16$ ). However, the specific indirect effect of selforiented perfectionism on demoralization through both socially prescribed perfectionism and appraisal was not significant ( $a 1 d b 2=0.0078,95 \% \mathrm{CI}[-0.0001,0.0256])$. Together, the variables accounted for $14.04 \%$ of the variance in demoralization $\left(R^{2}=.14\right)$. These results are presented in Figure 6.

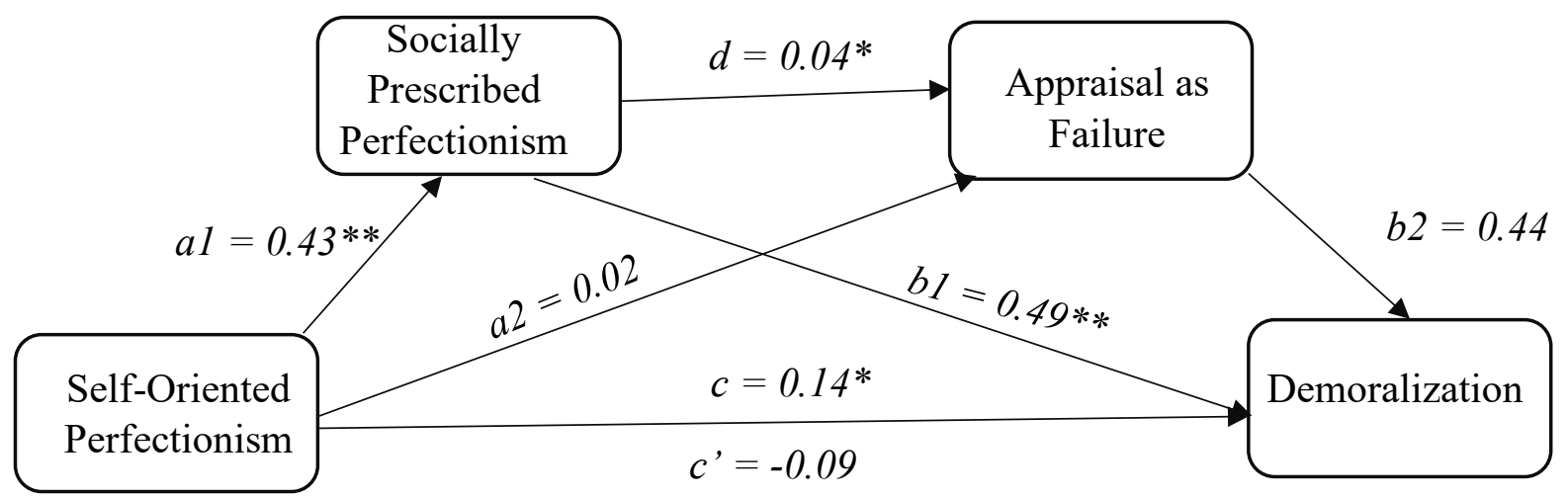

Figure 6. Statistical model of the proposed effect of self-oriented perfectionism on demoralization through socially prescribed perfectionism and appraisal. $*=p<.05, * *=p$ $<.0001$.

Main effect of negative life events on demoralization through appraisal. As reviewed, there was not support for an interaction between either dimension of perfectionism and negative life events in predicting demoralization through appraisal. However, there was moderate support for a main effect of negative life events on demoralization through appraisal, given the significant $b$ (see Figures 3, 4) and $c$ (see Hypothesis 2A) pathways. While the $a$ pathway was not significant (see Hypotheses 3Aii/3Bii), it is possible to have a significant indirect effect even with an insignificant $a$ pathway (Hayes, 2013). To test this supplemental hypothesis, mediation 
analyses were conducted with 10,000 bootstrapped samples (Preacher and Hayes, 2004). Model 4 in the PROCESS Macro (Hayes, 2013) was run to generate the point estimate and confidence interval of the mediation effect. Mediation was considered tenable if zero did not fall within the bounds of the confidence interval. Results of these analyses indicated that the indirect main effect of negative life events on demoralization through appraisal was not significant $(a b=$ $0.0037,95 \%$ CI [-0.1876, 0.1322]).

\section{Mediated effect of socially prescribed perfectionism on demoralization through}

individual appraisal components. Given the poor internal consistency of the measure of appraisal, it was conceivable that the three components of the measure (e.g. motivational relevance, falling short of standards, self-accountability) differentially related to socially prescribed perfectionism and demoralization, and that one or more components would mediate the relationship between socially prescribed perfectionism and demoralization when examined individually. To test this supplemental hypothesis, mediation analyses were conducted with 10,000 bootstrapped samples (Preacher and Hayes, 2004). Model 4 in the PROCESS Macro (Hayes, 2013) was run to generate the point estimate and confidence interval of the mediation effect. Mediation was considered tenable if zero did not fall within the bounds of the confidence interval. Results of these analyses indicated that the indirect main effect of socially prescribed perfectionism on demoralization through appraisal of falling short of standards was significant, but accounted for very little variance in the criterion $\left(a b=0.0214,95 \%\right.$ CI [0.0003, 0.0587], $R^{2}$ $=0.01)$. But see Fairchild et al. (2009). Socially prescribed perfectionism predicted a greater appraisal of falling short of standards ( $a$ path; $B=0.02, p<.01$ ), which was not significant in predicting greater demoralization ( $b$ path; $B=1.14, p=.06$ ). However, a significant $b$ pathway is not required for mediation to be present (Hayes, 2013). The direct pathway from socially 
prescribed perfectionism to demoralization, although decreased, remained significant ( $c$ ' path; $B$ $=0.4395,95 \% \mathrm{CI}[0.034,0.5755])$, suggesting partial mediation. Together, the variables accounted for $13.89 \%$ of the variance in demoralization $\left(R^{2}=.14\right)$. No other components of appraisal significantly mediated the relationship between socially prescribed perfectionism and demoralization. These results are presented in Figure 7.

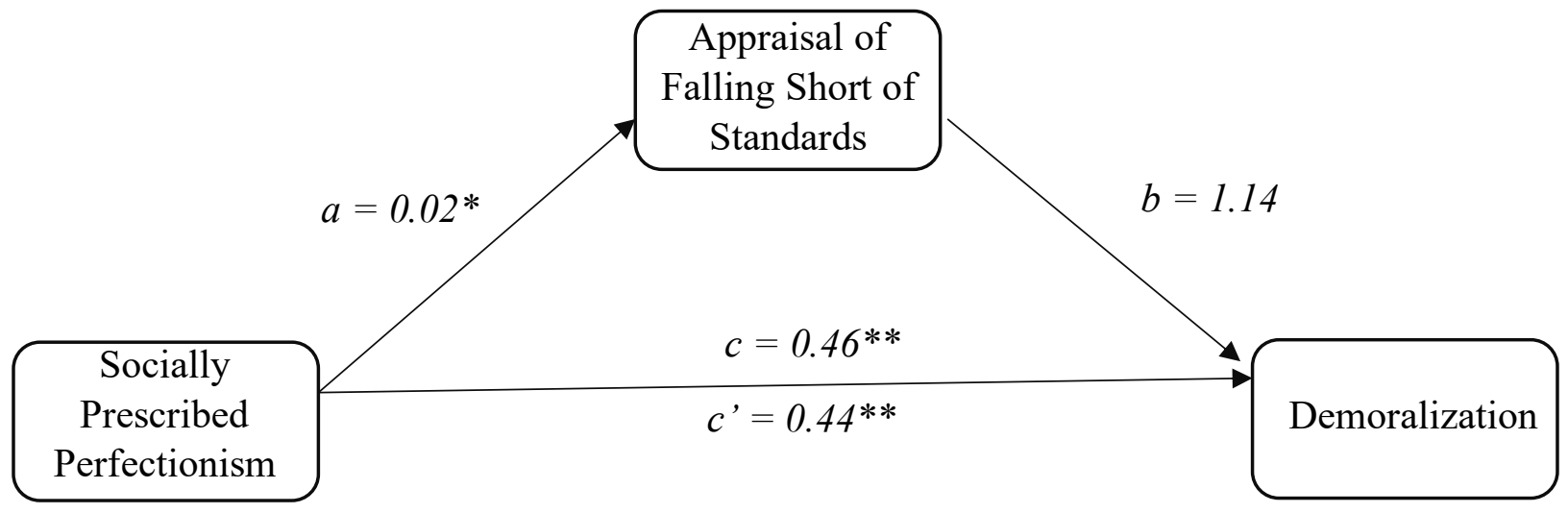

Figure 7. Statistical model of the proposed effect of socially prescribed perfectionism on demoralization through appraisal of falling short of standards. $*=p<.01,{ }^{*} *=p<.0001$.

\section{Mediated effect of self-oriented perfectionism on demoralization through individual}

appraisal components. It was likewise conceivable that the three components of the measure of appraisal would differentially relate to self-oriented perfectionism and demoralization, and that one or more components would mediate the relationship between self-oriented perfectionism and demoralization when examined individually. To test this supplemental hypothesis, mediation analyses were conducted with 10,000 bootstrapped samples (Preacher and Hayes, 2004). Model 4 in the PROCESS Macro (Hayes, 2013) was run to generate the point estimate and confidence interval of the mediation effect. Mediation was considered tenable if zero did not fall within the bounds of the confidence interval. Results of these analyses indicated that the indirect main 
effect of self-oriented perfectionism on demoralization through appraisal of falling short of standards was significant, but accounted for very little variance in the criterion $(a b=0.0211$, 95\% CI [0.0016, 0.0599], $\left.R^{2}=0.005\right)$. But see Fairchild et al. (2009). Self-oriented perfectionism predicted a greater appraisal of falling short of standards ( $a$ path; $B=0.01, p<.05$ ), which in turn predicted greater demoralization ( $b$ path; $B=1.61, p<.05$ ). The direct pathway from selforiented perfectionism to demoralization was $(p=0.06)$ no longer significant after accounting for the mediator $\left(c^{\prime}=0.1144,95 \%\right.$ CI $\left.[-0.0053,0.2341]\right)$, supporting full mediation. Together, the variables accounted for $3.60 \%$ of the variance in demoralization $\left(R^{2}=.04\right)$. No other components of appraisal significantly mediated the relationship. These results are presented in Figure 8.

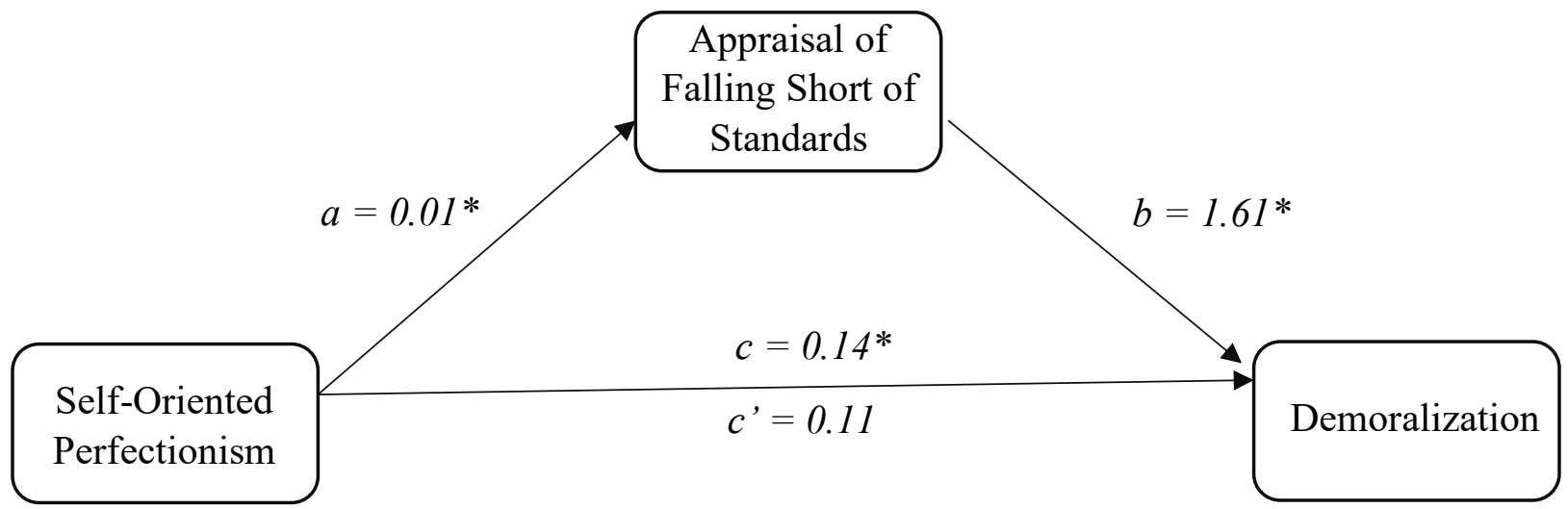

Figure 8. Statistical model of the proposed effect of self-oriented perfectionism on demoralization through appraisal of falling short of standards. $*=p<.05$. 


\section{Discussion}

The present study aimed to identify contextual factors which may particularly activate the maladaptive features of perfectionism, as well as to better understand the pathway through which these effects operate. It was proposed that perfectionism - self-oriented or socially prescribed would interact with negative life events to predict demoralization, through a pathway of appraisals of failure. As hypothesized, socially prescribed perfectionism uniquely related to demoralization. Experiencing a greater number of recent negative life events likewise predicted greater demoralization, as hypothesized. In contrast, self-oriented perfectionism did not relate to demoralization after controlling for the effects of socially prescribed perfectionism. Results indicated more complex relationship dynamics related to the collective effects of perfectionism, negative life events, and appraisal, on demoralization.

\section{Socially Prescribed Perfectionism as a Robust Risk Factor}

It was hypothesized that socially prescribed and self-oriented perfectionism would both uniquely relate to greater demoralization. Hypotheses were partially supported; while socially prescribed perfectionism demonstrated a unique relationship with demoralization, self-oriented perfectionism did not. However, these results are fairly consistent with the literature. That is, socially prescribed perfectionism appears to be more unequivocally linked to maladaptive outcomes such as poorer self-esteem, poorer achievement motivation, shame, guilt, dysthymia, depression, anxiety disorders, obsessive-compulsive disorder, anorexia nervosa, and borderline personality disorder (Enns \& Cox, 1999; Flett et al., 2004; Flett, Hewitt, Blankstein, \& O'Brien, 1991; Frost et al., 1993; Hewitt \& Flett, 1991a, 1991b; Klibert et al., 2005; Limburg, Watson, 
Hagger, \& Egan, 2017; Martin, Flett, Hewitt, Krames, \& Szanto, 1996; Shafran \& Mansell, 2001; Wyatt \& Gilbert, 1998), among others. In contrast, self-oriented perfectionism fails to demonstrate significant relationships with these concerning outcomes or does so to a lesser degree with less consistency in the literature. The finding that self-oriented perfectionism no longer relates to demoralization after controlling for socially prescribed perfectionism is particularly consistent with the results of Smith, Sherry, et al. (2018). They found that socially prescribed perfectionism predicted prospective increases in suicidal ideation and presence and number of suicide attempts; self-oriented perfectionism predicted only concurrent suicidal ideation, with this relationship becoming nonsignificant after controlling for socially prescribed perfectionism. The findings of the present study then, appear to support the broader literature indicating the particularly pernicious qualities of socially prescribed perfectionism.

The concerning effects of socially prescribed perfectionism were further demonstrated by its lack of a significant interaction with negative life events. Namely, it was hypothesized that socially prescribed perfectionism would interact with negative life events to predict demoralization, such that perfectionism would increasingly relate to demoralization as individuals experienced an increasing number of recent negative life events. This was not supported; while both socially prescribed perfectionism and negative life events significantly predicted demoralization as main effects, the interaction was not significant. This is somewhat consistent with prior literature. While very few studies have examined if Hewitt and Flett's $(1989,1991)$ dimensions of perfectionism interact with negative life events or stressors (Chang \& Rand, 2000; Dean \& Range, 1996; Dean et al., 1996; Dean \& Range, 1999; Flett et al., 1995; Hewitt et al., 1994; Hewitt et al., 1996; Hewitt et al., 2002; O'Connor 2010), about a third have found no support for an interaction (Dean \& Range, 1999; Hewitt et al., 1996; Hewitt et al., 
2002), with no clear differences in the conceptualization of negative life events across these studies. The present results suggest that perfectionism and negative life events may operate separately in parallel, rather than interactively influencing the effects of one another.

Alternatively, it may be that the concerning effects of socially prescribed perfectionism are not exacerbated by broadly defined stressors, such as negative life events, but are instead exacerbated by specific stressors. Namely, potentiation of socially prescribed perfectionism may require stressors that are interpersonal in nature.

While socially prescribed perfectionism's effects may not be exacerbated by negative life events broadly, negative interpersonal events specifically may demonstrate such an interaction. Although understudied, this proposal would be consistent with extant research. For instance, Wang, Wong, and $\mathrm{Fu}$ (2013) found that an interpersonal dimension of perfectionism comparable to socially prescribed perfectionism (here labeled family discrepancy) interacted with interpersonal suicide risk factors (i.e. perceived burdensomeness and thwarted belongingness; Joiner, 2005) to predict suicidal ideation. Likewise, socially prescribed perfectionism interacted with loneliness to predict greater symptoms of anxiety and depression (Chang, Sanna, Chang, \& Bodem, 2008). That is, individuals with elevated socially prescribed perfectionism may be more vulnerable to its concerning effects when interpersonal difficulties are also experienced. That socially prescribed perfectionism may require specific stressors to moderate its effects is particularly supported by findings from Hewitt and Flett (1993). They found that, while selforiented perfectionism only interacted with achievement stressors to predict depression, socially prescribed perfectionism only interacted with interpersonal stressors. This would again suggest that specific, likely interpersonal, triggers are required to potentiate socially prescribed perfectionism's effects. That is, for negative life events to be increasingly aversive as individuals 
are increasingly elevated in socially prescribed perfectionism, it may be necessary for these events to be interpersonal in nature, given that socially prescribed perfectionism is itself defined by interpersonal concerns. Thus, individuals with elevated socially prescribed perfectionism may be particularly concerned about, and affected by, negative life events which threaten to deteriorate interpersonal relationships. Those events which do not imply interpersonal consequences may be experienced similarly regardless of one's level of socially prescribed perfectionism. Taken in consideration with the present results, it is clear that more research is needed to determine if, or perhaps when, the deleterious effects of socially prescribed perfectionism are potentiated by activating environmental factors. The proposal that negative interpersonal events may be experienced as particularly aversive in those with elevated socially prescribed perfectionism presents as a compelling future direction. Importantly, the current study suggests that socially prescribed perfectionism merits concern even in the relative absence of such factors.

The maladaptive nature of socially prescribed perfectionism can be further understood through examining the magnitude of its effect on demoralization. Namely, socially prescribed perfectionism accounted for considerably more variance in the criterion. While negative life events accounted for $3.90 \%$ of the variance in demoralization, socially prescribed perfectionism accounted for $12.00 \%$, even after controlling for any variance explained by self-oriented perfectionism. Thus, not only do negative life events fail to potentiate the effect of socially prescribed perfectionism on demoralization, but even as a main effect they demonstrate a relationship that is approximately one-third the magnitude of that demonstrated by socially prescribed perfectionism. This is somewhat unexpected, given prior literature showing that experiencing a greater number of recent negative life events predicts worse physical health, 
including mortality and sudden cardiac death (Lantz, House, Mero, \& Williams, 2005; Rahe \& Lind, 1971; Wyler, Masuda, \& Holmes, 1971); substance misuse, poorer self-esteem, hopelessness, anxiety, depressive symptomatology, presence and severity of NSSI, presence and severity of suicidal ideation, duration of acute suicidal crises, and odds of a suicide attempt (Bagge, Glenn, \& Lee, 2013; Covault et al., 2007; Dean et al., 1996; Flett et al., 1995; Joiner \& Rudd, 2000; Madge et al., 2011; O'Connor et al., 2010; Osvath, Vörös, \& Fekete, 2004); and prospective suicide attempts (Yen et al., 2005). Accordingly, negative life events appear to merit concern in their own right; however, socially prescribed perfectionism may present as an even more pressing mental health concern, at least as related to demoralization.

It is unclear why socially prescribed perfectionism, a stable personality trait, would be more predictive of acute demoralization relative to recent negative life events; that is, it is not necessarily intuitive that an individual who consistently presents with high concern for perceived standards, expectations, and pressures from others would be at greater risk for demoralization than an individual who had, say, recently broken up with a partner, failed an academic course, or experienced direct or passive victimization, as the present results would suggest. Nonetheless, this may speak to the nefarious, and overlooked, effects of socially prescribed perfectionism. Consider, for instance, that negative life events may be noticed by, and accordingly generate support from, one's social environment. Someone who has recently experienced a personal injury or illness, who has lost a close friend, or who has gotten in a car accident, may receive greater support and concern from a social support network (e.g. Antonovsky, 1979; Hallaraker, Arefjord, Havik, \& Mæland, 2001). Furthermore, these events may prompt meaning-making, insight, or other adaptive appraisal attempts (McFarland \& Alvaro, 2000; Tugade \& Fredrickson, 2011). Likewise, individuals may be able to recognize that they have experienced a negative life 
event and respond with other increased coping efforts (Folkman \& Lazarus, 1980, 1985;

Folkman, Lazarus, Gruen, \& DeLongis, 1986). In contrast, socially prescribed perfectionism is unlikely to effect such protective factors, for at least two reasons.

First, socially prescribed perfectionism lacks any activating event to prompt such external or internal coping. This diluted, relatively enduring, stable (Hewitt \& Flett, 1991b) personality trait may be conceptualized as white noise, operating more or less undetected in the background. Individuals high in socially prescribed perfectionism may - at times correctly (Smith et al., 2017) - perceive external standards as veridical, may have little insight into their perfectionism (Sironic \& Reeve, 2012), and may have long since taken such perceived pressures for granted, effectively failing to "hear" the white noise that saturates their reality. Thus, if individuals do not recognize that they perceive disproportionately high standards or external pressures from others, that they distort and disproportionately fear the consequences of failure, or that they discount and feel less satisfaction from the progress they have made, among other features, it is difficult if not impossible to reappraise or otherwise cope with the pernicious features of socially prescribed perfectionism. This proposal needs far greater research to determine to what extent individuals have insight into their own experiences of perfectionism. Preliminary investigations may wish to begin with assessing the effects of psychoeducation of perfectionism on individuals' abilities to recognize, and respond compassionately to, their own perfectionistic tendencies.

Second, perfectionism is unlikely to generate increased social support, not only for its diffuse nature, but also because it is often viewed favorably (e.g. Schuler, 2000; Silverman, 1995; 2007). Those effects of perfectionism which are likely to be noticed externally (i.e. high achievement striving, high standards, ambitious goals) may garner reinforcement (e.g. Slade \& Owens, 1998), in the form of praise or admiration, rather than empathy, support, or assistance 
from the environment. Indeed, an individual who highly values a perfectionistic self-presentation (Chen et al., 2015; Mushquash \& Sherry, 2012) may be viewed as more self-sufficient and capable (e.g. apparent competence) and less likely to need support. Simultaneously, socially prescribed perfectionism is associated with negative social interactions (Flett, Hewitt, Garshowitz, \& Martin, 1997) and socially distant characteristics (Hill, Zrull, \& Turlington, 1997). In this way, socially prescribed perfectionism may actually attenuate the responsiveness of one's social network. These present as only two possible pathways by which socially prescribed perfectionism, a stable personality trait, may predict concerning outcomes such as demoralization to a much greater degree than recent negative life events.

While socially prescribed perfectionism appears to be a concerning risk factor for maladaptive outcomes such as demoralization, far greater research will be needed to confirm and clarify this relationship. Extant research suggests that socially prescribed perfectionism may be particularly pernicious for its social consequences (e.g. increased interpersonal conflict [Mushquash \& Sherry, 2012], anticipatory fear of interpersonal consequences of failure [Conroy, Kaye, \& Fifer, 2007], competition with peers [Neumeister \& Finch, 2006]) and relation to burnout and exhaustion (Childs \& Stoeber, 2010). However, it is possible that the present results are an artifact of method effects (Spector, 2006); namely, both socially prescribed perfectionism and demoralization were assessed with self-report Likert-style scales, and some shared variance may simply be a result of this similarity in measurement. However, given that all study variables were operationalized similarly, it would not be expected that the correlation of socially prescribed perfectionism and demoralization would uniquely be affected by this methodological overlap. Furthermore, research suggests that the inflationary effect of common method variance is almost entirely negated by the attenuating effect of measurement error (Lance, Dawson, 
Birkelbach, \& Hoffman, 2010). Alternatively, the correlation may be inflated by similarity in the constructs; the Demoralization Scale's "Sense of failure" subscale (Kissane et al., 2004), which includes items such as "I am proud of my accomplishments (reverse-scored)" and "I cope fairly well with life," may be conceptually similar to perfectionism. However, it is unlikely that this would uniquely inflate the correlation between socially prescribed perfectionism and demoralization, and not the correlation between self-oriented perfectionism and demoralization; that is, the items comprising the subscale do not appear specific to socially prescribed perfectionism.

\section{The Role of Self-Oriented Perfectionism}

As reviewed, self-oriented perfectionism did not uniquely relate to demoralization, after accounting for the effects of socially prescribed perfectionism. This is contrary to study hypotheses, but nonetheless relatively consistent with prior literature showing a primarily benign effect of self-oriented perfectionism (e.g. Blankstein, Lumley, \& Crawford, 2007; Dean \& Range, 1999; Dean et al., 1996; Enns \& Cox, 1999; Flett et al., 1995; Flett et al., 2004; Flett, Hewitt, Blankstein, \& O'Brien, 1991; Frost et al., 1993; Hewitt \& Flett, 1991a, 1991b; Klibert et al., 2005; Limburg, Watson, Hagger, \& Egan, 2017; Martin, Flett, Hewitt, Krames, \& Szanto, 1996; Shafran \& Mansell, 2001; Smith, Sherry, et al., 2018; Wyatt \& Gilbert, 1998). Throughout the literature, self-oriented perfectionism appears to demonstrate less significant, less consistent, and less robust relationships with maladaptive outcomes, relative to socially prescribed perfectionism. However, much of this research has neglected to consider the overlap in selforiented and socially prescribed perfectionism, missing a compelling pathway by which selforiented perfectionism may become nefarious. 
While the two appear to represent distinct factors (e.g. (Bieling et al., 2004; Frost et al., 1993), they nonetheless correlate fairly highly (e.g. $r=.51, p<.01$ in the present study). It is possible then, that self-oriented perfectionism may be an indicator of developing, or concurrently experiencing, socially prescribed perfectionism. Consider, for instance, the possible role of geneenvironment correlations ( $r G E$; e.g. Knafo \& Jaffee, 2013). Individuals who demonstrate a high degree of "benign" achievement striving (i.e. self-oriented perfectionism) may seek out highachieving environments (active $r G E$; Knafo \& Jaffee, 2013), such as academic clubs, organizations, and societies; advanced courses; Ivy League or otherwise elite universities; and competitive extracurricular activities, among others. As these individuals select into increasingly high achieving cohorts of peers, they may increasingly infer high standards from their environment. They may likewise begin to evoke high standards from their environments (evocative $r G E$; Knafo \& Jaffee, 2013). As reviewed, high achievement striving may be reinforced by close others; simultaneously, high standards and expectations (e.g. from parents, teachers, or peers) and the accompanying praise may be reinforced by the individual's successfully meeting those standards. Put simply, a parent may notice her child performing well in school and may communicate to the child the family's valuing of, and happiness with, his current success. This child may then perceive that doing well in school is important and may accordingly continue or increase high effort in schoolwork. In this way, the child is reinforced for high achievement striving by praise, while the parent is reinforced for praising high achievement by the child's continued or increased achievement striving. Thus, it is possible that relatively benign, self-motivated perfectionism (i.e. self-oriented perfectionism) may develop into its more pernicious counterpart through these or other pathways. 
To test the possibility that self-oriented perfectionism may increase risk for socially prescribed perfectionism, in turn leading to increased risk for the myriad reviewed maladaptive outcomes, a supplemental mediational analysis was run. Results suggested that self-oriented perfectionism predicted greater demoralization and appraisals of failure, with both of these pathways fully mediated by socially prescribed perfectionism (see Figure 6). Thus, at lower levels of self-oriented perfectionism, it appears that individuals are unlikely to have elevated levels of socially prescribed perfectionism and are accordingly unlikely to experience concerning outcomes such as those included in this model. However, as individuals increasingly endorse the arguably benign experiences of self-oriented perfectionism, they may be more likely to also increasingly endorse socially prescribed perfectionism, and thus present as at increasing risk for the latter's maladaptive effects. Considered with results of testing Hypothesis 1B - namely, that self-oriented perfectionism demonstrated no significant unique effect on demoralization after accounting for socially prescribed perfectionism - it appears that self-oriented perfectionism in fact may be concerning, to the extent that it operates through a pathway of co-occurring socially prescribed perfectionism. Accordingly, even those individuals who appear to set high standards for themselves, or who appear to be internally motivated in their achievement striving, may merit further assessment of their functioning and well-being.

While these results offer a compelling pathway for additional investigation, it is again possible that other explanations for the present findings are tenable. Consider again the possibility of methodological confounding. That is, several items on the socially prescribed perfectionism subscale of the MPS (Hewitt \& Flett, 1990) allude to consequences of failure (e.g. upsetting close others, being subject to negative evaluation), whereas corresponding items on the self-oriented perfectionism subscale appear to only describe the existence of high standards. 
Accordingly, it is conceivable that self-oriented perfectionism would exhibit a stronger relationship with demoralization, perhaps comparable to that of socially prescribed perfectionism, if its operationalization included the perceived consequences of failure. In this vein, it is likewise possible that the effects of both self-oriented and socially prescribed perfectionism on demoralization operate through a pathway of perceived consequences of failure, resulting in the present mediation results. That is, rather than self-oriented perfectionism, the first step in the pathway may instead simply be the existence of high standards, whether set or perceived; these may then lead to maladaptive outcomes through the anticipated consequences of failure. To pursue this possibility, future researchers will need to clearly define perfectionism and discriminate it from perceived consequences of failure in its operationalization. As it stands, few items on the socially prescribed perfectionism subscale included anticipated consequences, so it remains unclear if, or to what extent, this could be an alternate explanation of study results.

In addition to this possibility, the present results are further limited by their crosssectional nature. Longitudinal research is severely needed to determine how these distinct facets of perfectionism may interact, develop, and change over time. It will be important to determine if self-oriented perfectionism consistently precedes socially prescribed perfectionism, or if the reverse is ever documented. If such a temporal pattern is supported, it will likewise be important to identify those factors (e.g. depleted social support, excessive reassurance seeking, unstable sense of self, etc.) which may increase risk of self-oriented perfectionism contributing to the development of socially prescribed perfectionism, or the pathways by which this occurs. Furthermore, support for the proposed pathway from self-oriented perfectionism to socially prescribed perfectionism would be strengthened by research examining if, or how, self-oriented perfectionism influences choice of one's environment, particularly one's social environment. If 
there is empirical support for the role of self-oriented perfectionism in selecting into or evoking environments which reinforce unrealistic standards and achievement striving, this would strengthen the directional pathway. Likewise, far greater research is needed on how these individuals' social environments respond to, and potentially reinforce, their perfectionistic behaviors and attitudes. Clarification of the temporal and emergent nature of these processes, and identification of potentiating factors, would allow for essential early intervention, breaking the link between the two dimensions of perfectionism and the potential negative consequences that can follow.

\section{Differential Mediating Effects of Specific Appraisals}

It was hypothesized that perfectionism's effects would be mediated by appraisals of negative life events as failures — that is, as important to oneself (motivational relevance; Smith \& Lazarus, 1990), as falling short of one's standards (e.g. motivational incongruence; Smith \& Lazarus, 1990), and as something for which one is responsible (e.g. internal attribution, personal accountability; Abramson, Seligman, \& Teasdale, 1978; Smith \& Lazarus, 1990). While neither dimension of perfectionism was mediated by composite appraisals of failure in their relationship with demoralization, both were mediated by one facet of these appraisals - appraising a negative life event as falling short of standards (motivational incongruence). In contrast, neither motivational relevance nor personal accountability significantly mediated perfectionism's effects. The present results suggest that perfectionism, both socially prescribed and self-oriented, is particularly likely to effect appraisals of a negative life event falling short of one's standards, which in turn may predict greater demoralization. While preliminary, this presents as an important contribution to a neglected area of the literature. 
Very few studies have examined how appraisal may operate in contributing to the pernicious effects of perfectionism, particularly in the context of negative life events. As reviewed, extant studies make the assumption that negative life events are increasingly appraised as instances of personal failure as one is increasingly perfectionistic, despite neglecting to measure appraisal or test this assumption (e.g. Dean \& Range, 1996, 1999; Dean et al., 1996; Dean, Range, \& Goggin, 1996; Hewitt \& Flett, 1993; Hewitt et al., 1996; Hewitt, Flett, and Weber, 1994). Furthermore, very few studies have employed the framework proposed by Smith and Lazarus (1990). Of the relevant studies which have employed this framework, appraisal components have overwhelmingly been conceptualized as operating collectively (Coo, Milgrom, Kuppens, \& Trinder, 2015; Lowe \& Bennett, 2003; Smith \& Lazarus, 1990; Smith et al., 1993; Smith \& Lazarus, 1993); the unique influence of any specific appraisal component has largely been neglected. Nonetheless, research exists linking motivational incongruence to prospective distress and depression (Coo et al., 2015), as well as to guilt, anger, and sadness (Bennett, Lowe, \& Honey, 2003). Thus, appraisals of falling short of standards appear to influence similar outcomes, but necessitate far greater research. Even conceptualized collectively, appraisals remain understudied in relation to negative life events and perfectionism.

While no known study has previously examined the transactional effects of negative life events, perfectionism, and failure appraisals, the present results are surprising when considered alongside relevant appraisal theories (e.g. Abramson et al., 1978; Smith \& Lazarus, 1990). These models propose that the extent to which any external or internal stimulus is distressing, demoralizing, or defeating depends, at least in part, on how this stimulus is appraised. Smith and Lazarus (1990, p. 616) write, "How a given individual reacts emotionally to an encounter depends on an evaluation of what the encounter implies for personal well-being which is what 
'appraisal' means in our usage. ... That is, if we know how a person evaluates the relationship with the environment, we can predict that person's emotional reaction." The lack of significant mediation is also surprising when considering studies of appraisal more broadly, which have found some support for such a pathway. Prior research has found that positive and negative interpersonal events may predict distress and well-being through a pathway of appraisal (Newsom, Rook, Nishishiba, Sorkin, \& Mahan, 2005). Likewise, appraisals of control may mediate the relationship between negative life events and life satisfaction (Ash \& Huebner, 2001). Appraisals of negative life events mediated the relationship between genetic risk for depression (i.e. 5-HTTLPR) and current depressive symptoms (Conway et al., 2012). The present results are nonetheless consistent with some extant literature; for instance, appraisals of life events did not mediate their effect on behavioral responses (Jackson, Kim, \& Delap, 2007). Further, the effect of negative life events on internalizing and externalizing problems was not mediated by appraisals of control (Haine, Ayers, Sandler, Wolchik, \& Weyer, 2003). Ultimately, far greater research is needed on the degree to which the psychological consequences of negative life events are explained by the way in which these events are appraised, particularly as this dynamic process relates to individuals with elevated perfectionism.

While the lack of overall mediation was somewhat inconsistent with extant theory and literature, this may speak to the value of considering appraisal components individually. That is, given the likely distinct nature of the facets of appraisal (e.g. Cronbach's $\alpha$ of 0.56 in the present sample), it is conceivable that not all are subject to perfectionism's influence. While perfectionism appears to increase appraisals of events as falling short of standards (motivational incongruence), it may be less likely to increase individuals' appraisals of the event as their fault (self-accountability), or as important to themselves (motivational relevance). In other words, 
perfectionism alone is unlikely to determine the extent to which individuals appraise an event as important to themselves; similarly, it is unlikely that perfectionism would create some systematic vulnerability wherein individuals perceive all negative life events to be their own fault. While it is conceivable that perfectionism could contribute some variance to appraisals of importance and responsibility, there are likely many other factors which overwhelm this contribution. Instead, individuals with elevated perfectionism may care about, and take responsibility for, those negative life events which relate to their specific values, goals, and objectives. Thus, perfectionism's influence on appraisals of responsibility and importance may not be systematic and predictable; these appraisals may instead be dependent on many other extant factors.

In contrast, the present results suggest that both self-oriented and socially prescribed perfectionism increase vulnerability to appraising events as falling short of standards, which in turn may lead to greater demoralization. This conceptual pathway makes intuitive sense, in that perfectionism is characterized by unrealistically high standards (Burns, 1980; Frost, Marten, Lahart, \& Rosenblate, 1990; Hamachek, 1978; Hewitt \& Flett, 1989), fear of falling short of standards (e.g. Burns, 1980; Conroy, Kaye, \& Fifer, 2007), and an overly critical evaluation of progress towards standards (Grzegorek, Slaney, Franze, \& Rice, 2004; Rice, Ashby, \& Gilman, 2011). Accordingly, this mediational pathway is logically consistent with expectations created by perfectionism. Further, as previously reviewed, this would be consistent with the Cry of Pain theory of suicide (Williams, 2001) and escape theories of suicide (e.g. Baumeister, 1990). That is, the way in which any given situation is cognitively appraised will influence the individual's response to it; if individuals with elevated perfectionism encounter a situation, behavior, etc. and appraise it as falling short of standards, this appraisal, rather than or in addition to the event itself, may be experienced as unbearably aversive, defeating, and demoralizing, as suggested by 
the present results. Indeed, a large body of literature supports such a link between negative appraisals and affect (e.g. Baum, Fleming, \& Singer, 1983; Cohen, Gunthert, Butler, O’Neill, \& Tolpin, 2005; Folkman, Lazarus, Dunkel-Schetter, DeLongis, \& Gruen, 1986; Lee-Flynn, Pomaki, DeLongis, Biesanz, \& Puterman, 2011). Accordingly, this resulting affective state may then lead to suicidal crisis through a pathway of entrapment and little hope for escape (e.g. Baumeister, 1990; Williams, 2001). Additional investigation will be required to determine the reliability of these findings; nonetheless the mediating role of falling short of standards is consistent with extant empirical literature. Although unexamined beyond the present study, related research finds socially prescribed perfectionism to be predictive of prospective depressive symptoms, mediated by appraisals of falling short of others' standards (Smith et al., 2013; Smith, Sherry, \& McLarnon, et al., 2018). Similarly, the previously reviewed relationship between perfectionism and procrastination (Martin et al., 1996; Neumeister, 2004; Saddler \& Sacks, 1993; Sheykhi et al., 2013; Sudler, 2014), which itself may be conceptualized as a form of avoidance/escape, has been found to be mediated by a subjective sense of falling short of standards (Smith, Sherry, Saklofske, \& Mushqaush, 2017). Further, perceptions of falling short of standards may be associated with greater negative affect and decreased positive affect and emotional stability (Mitchelson, 2009). Despite this preliminary support, the role of appraisals of falling short of standards broadly has been severely neglected in the literature, highlighting the need for further research.

\section{Limitations}

While the results of the present study serve to advance understandings of perfectionism's maladaptive qualities and consequences, they should be interpreted in light of several limitations. 
Cross-sectional correlational design. First, the cross-sectional nature of the study precludes understanding of the causal nature of the relationships between the study variables. Thus, while the present study theorizes that socially prescribed perfectionism leads to demoralization, the reverse may prove to be true. However, to account for this limitation, all models were run in reverse direction, and regression coefficients and effect sizes were examined; the regression pathways and indirect effects were consistently greater in the hypothesized direction than they were in the reverse. Further, this directionality makes greater conceptual sense. That is, perfectionism appears to be a fairly stable, enduring personality trait (e.g. Hewitt \& Flett, 1991b), whereas demoralization reflects an acute state (Catanese et al., 2009; Kissane et al., 2004; Marsa et al., 2017; Zanicotti, Perez, \& Glue, 2012), making it more plausible for the former to influence the latter. Likewise, the measure of demoralization referred to the previous two weeks, whereas the measure of perfectionism was not bound by a particular time frame; it is unlikely, though possible, that an aversive affective state over the previous two weeks would meaningfully influence this stable construct which encompasses a far vaster timeframe. Nonetheless, longitudinal research will need to be conducted to document the temporal emergence of these phenomena. Further, causality may be better understood through experimental rather than correlational design; for instance, researchers may wish to experimentally induce demoralization and determine what effect, if any, this has on reported levels of socially prescribed perfectionism. The present results set the foundation for these and other studies.

Use of self-report survey data. There are several potential limitations posed by the use of self-report survey data. Namely, self-report measures may be particularly vulnerable to inaccurate responding (e.g. Wright, 2005) and social desirability effects (Arnold \& Feldman, 
1981). Use of multiple informants/behavioral observations may provide more compelling assessments of the constructs under study. Nonetheless, the effects of careless responding are unlikely to have meaningful impacts on the present results; several attention checks were integrated into the study surveys, and individuals who failed attention checks were removed from the present sample. As reviewed, these individuals were more likely to be male and to report never having had a mental health disorder, limitations which may be addressed by including more diverse samples in future investigations. Likewise, while social desirability or other processes may constrain the quality of data obtained via self-report measures, considerable research suggests greater disclosure on such measures relative to other methodologies (e.g. inperson interviews), particularly for potentially sensitive topics (Corrigan \& Watson, 2002; Kaplan, Asnis, Sanderson, Keswani, de Lecouna, \& Joseph, 1994). Participants may be especially likely to respond honestly given the anonymous nature of the present study. And, although data from multi-informants or behavioral observations would be desirable, self-report data tends to be more predictive of internalizing processes (e.g. perfectionism, demoralization, appraisal), relative to observer reports (e.g. Manassis, Tannock, \& Monga, 2009). Finally, the use of self-report surveys facilitated the data collection process, allowing for a larger sample with little participant demand. Indeed, aside from the Scale for Assessing Appraisal Components, the study variables had no missing data. Thus, while the data from self-report measures may be limited by careless responding, social desirability, or lack of observer reports, this methodology nonetheless may have facilitated greater disclosure, more accurate representation of these internalizing processes, and reduced participant demand. Still, observer reports of these internalizing processes may be insightful in considering how individuals manifest perfectionism behaviorally, as well as how this is perceived by those in the social environment. In particular, 
this might be useful for better understanding the process by which individuals high in socially prescribed perfectionism evoke or reinforce high standards from their environment. Furthermore, this may be illustrative in studying the Perfectionism Social Disconnection Model (PSDM; Hewitt, Flett, Sherry, \& Caelian, 2006), which proposes that individuals with elevated socially prescribed perfectionism inadvertently deteriorate social connections (e.g. through hostile attribtuions, rejection sensitivity, attempting to present as overly perfect, etc.).

College sample. The use of a college sample limits generalizability of the present findings. The results may not generalize to middle or high school students, non-college young adults, or older adult populations, given different normative developmental and social milestones. For instance, while both high school and college students may face standards related to academics, the latter are also often faced with expectations regarding autonomy, career development, financial stability, and other concerns. Likewise, while young adults broadly may progress through the same stages of life, those enrolled in college may face expectations or standards unique from those in the workforce. Consider that individuals who elect to attend a college or university may be more likely to perceive or set high standards (e.g. expectations to do well enough academically to be accepted into college, expectations to attend and complete college, etc.). However, this distinct nature of a college sample also makes it particularly appropriate for the present study, given heightened perfectionism in college samples (e.g. more than half of all college students demonstrate elevated perfectionism; Grzegorek et al., 2004).

The sample is further limited by its recruiting students currently enrolled in a psychology course; as with college enrollment, one's major may influence the degree to which high standards are imposed, set, or perceived. For instance, it is plausible that students electing into STEM (e.g. engineering, pre-med coursework, etc.) majors may be particularly vulnerable to 
self-oriented or socially prescribed perfectionism given the rigorous culture of these and related fields (for review, see Rice, Richardson, \& Ray, 2016). Nonetheless, students enrolled in a psychology course may represent a diverse background of majors and programs of study, as an introductory psychology course is generally required as part of general education curriculum.

It is important to note that the present study took place at the University of South Florida. There has been little study on how rates of perfectionism among college students vary per geographic region, acceptance rates, college ranking, etc. Accordingly, it is difficult to state with confidence how the present results may generalize to programs with more or less rigorous academic entry and performance standards. Nonetheless, existing research supports crossuniversity consistency in the construct, even across distinct geographic regions (e.g. Rice, Ashby, \& Slaney, 2007).

Considering specific demographics, participants were primarily young (mean age $=21.18$, $\mathrm{SD}=4.10)$, female $(82 \%)$, heterosexual $(83 \%)$, and cisgender $(98 \%)$. Nonetheless, gender was tested as a potential covariate and was found to be unrelated to levels of perfectionism, consistent with prior research (e.g. Hewitt \& Flett, 1991b). While generalizability may be severely limited when sampling from a population that is primarily white, educated, industrialized, rich, and democratic (WEIRD; e.g. Henrich, Heine, \& Norenzayan, 2010), the present sample nonetheless demonstrated moderate diversity. With consideration to race/ethnicity, only $44 \%$ of the sample was Caucasian; many participants were multiracial (16\%), Hispanic/Latinx (14\%), Asian/AsianAmerican (9.7\%), Black/African-American (8.4\%), Arabic/Middle Eastern (3.8\%), or another race $(2.8 \%)$. Further, $28 \%$ of participants were first-generation college students, meaning they were the first in their family to attend college, and participants were nearly equally distributed across year in school. Additionally, aside from the Demoralization Scale, means obtained in the 
present sample were not significantly different from those obtained in prior research, as reviewed. Still, future researchers are encouraged to determine how the results generalize to different samples (e.g. male, non-student, transgender, different developmental stage, etc.).

\section{Summary and Future Directions}

Socially prescribed perfectionism as a robust risk factor. With these limitations in mind, the study nonetheless presented with several strengths. This is the first known study to propose and test a relationship between perfectionism and demoralization. Likewise, this study uniquely considered the independent effects of socially prescribed and self-oriented perfectionism. The present results supporting the relationship between socially prescribed perfectionism and demoralization may serve as a foundation for more complex research hypotheses and designs moving forward. Further, this study is one of few to consider contextual factors (e.g. negative life events) which may be implicated in activating or potentiating perfectionism's maladaptive effects; importantly, it is the first known study to propose and test a pathway by which these contextual factors may influence perfectionism's effects.

The lack of interaction between perfectionism and negative life events in the present results highlights the need for additional research into if, or when, contextual factors may be particularly relevant, and through what mechanisms. Accordingly, an important future direction will be determining if there are factors which potentiate socially prescribed perfectionism's relationship with maladaptive outcomes. That is, would this dimension of perfectionism ever be a greater risk factor, or are contextual factors always irrelevant? A compelling possibility is that socially prescribed perfectionism may be exacerbated by specific negative life events. In short, individuals with elevated socially prescribed perfectionism who encounter negative life events related to domains for which they have set high standards, may demonstrate greater 
demoralization or other maladaptive outcomes. Negative life events broadly, however, may be unlikely to heighten perfectionism's pernicious qualities. Thus, if the primary object of one's high standards is, say, academic performance, the experience of unrelated negative life events (e.g. car crash, fight with a friend) may be experienced equivalently regardless of one's level of perfectionism. In contrast, for such individuals, failing a test may be experienced as increasingly aversive as they are increasingly perfectionist. Research is first needed to document to what extent "categories" of standards (e.g. academic, occupational, peer, family, body image, athleticism, etc.) are tenable. If support is found for this conceptualization, the potentiating effects of domain-specific negative life events may be tested.

Perhaps more importantly, researchers may wish to explore those factors which may be protective for individuals with elevated perfectionism. Here, researchers may specifically wish to consider social and interpersonal constructs. Given that socially prescribed perfectionism is characterized by its interpersonal nature (e.g. Hewitt \& Flett 1989, 1991b), anticipation of interpersonal consequences for failure (Conroy, Kaye, \& Fifer, 2007), frequency of negative social interactions (Flett et al., 1997; Hewitt et al., 2006; Mushquash \& Sherry, 2012), and an isolating perfectionistic self-presentation (Chen et al., 2015; Mushquash \& Sherry, 2012), perceptions of, for example, strong, unconditional social support may be particularly protective for these individuals.

A related and surprising finding of the current results was the comparative effects of perfectionism and negative life events on demoralization. Specifically, socially prescribed perfectionism was found to account for far more variance in demoralization than the experience of these recent, distressing life events. As reviewed, however, it is possible that negative life events catalyze meaning making, social support, coping, and other adaptive responses which are 
not activated by the stable, enduring, and subtle trait of socially prescribed perfectionism. Future researchers are encouraged to test this postulation, for instance by collecting momentary assessments from individuals with varying levels of perfectionism as they encounter negative life events or even daily hassles. Alternatively, researchers may wish to implement an intervention consisting of psychoeducation on perfectionism with vulnerable samples, to determine if insight into one's perfectionism may motivate coping, support seeking, and other responses, and if these responses indeed reduce perfectionism's association with maladaptive outcomes.

The role of self-oriented perfectionism. As stated, a strength of the present study was the examination of unique, independent relationships between both dimensions of perfectionism and study variables. Consistent with prior research, self-oriented perfectionism did not significantly predict demoralization after controlling for socially prescribed perfectionism. However, the present study extends this research in an important way; this is the first known study to propose that self-oriented perfectionism may increase vulnerability for, and thus operate through a pathway of, socially prescribed perfectionism. Indeed, while more research is needed, the present results suggest that this directional pathway may be tenable. This is not an inconsequential finding. If future research further supports this postulation, it would imply that self-oriented perfectionism — long considered a benign, or even adaptive, dimension of perfectionism - may in fact be quite concerning, to the extent that it elevates vulnerability for its maladaptive counterpart. Accordingly, it will be important for longitudinal research to be conducted, to better clarify the temporal emergence and dynamics of these dimensions of perfectionism.

Along this line, future researchers are also encouraged to better explicate those factors which discriminate self-oriented from socially prescribed perfectionism. That is, aside from the 
source of one's standards, how does one dimension of perfectionism differ from the other, and which of these qualities are particularly integral in effecting socially prescribed perfectionism's maladaptive nature? Thus, researchers are encouraged to identify and parse those qualities comprising either dimension of perfectionism and examine how they relate to outcomes of interest. Among other qualities, researchers may wish to consider motivation for achievement striving (e.g. intrinsic relative to extrinsic, curiosity relative to fear of failure), insight into and satisfaction with progress towards goals, perceived or veridical consequences for failing to meet standards, and ability to relinquish unrealistic goals and form new ones. Better identification of the "active ingredient(s)" of socially prescribed perfectionism would facilitate more targeted intervention efforts.

Appraisals as a mechanism. This study also contributed to a severely neglected area of the literature; namely, there is little research on the transaction between perfectionism and specific appraisals. While this area still needs substantial additional research, the present results offer insight into potentially relevant appraisals to consider (i.e. falling short of standards). Future research should further test this preliminary finding. Additionally, it may be compelling to consider the object of appraisal. That is, it is possible that standards are domain-specific (e.g. individuals may specifically have high standards only for academic achievement or only for family roles, etc.); if so, socially prescribed perfectionism may elevate vulnerability for appraising negative life events relevant to these specific domains as falling short of standards, rather than for appraising any negative life event as falling short of standards. Such an approach may better capture the processes by which socially prescribed perfectionism effects concerning outcomes such as demoralization. 
Given the lack of mediation by overall appraisals, an important future direction will be clarifying additional mechanisms by which perfectionism leads to maladaptive outcomes, such that prevention and intervention efforts may be implemented upstream. As suggested by the present literature review, there are many plausible alternative mechanisms (e.g. social/interpersonal factors, burnout, exhaustion, self-handicapping behaviors, psychopathology broadly). Alternatively, researchers may consider testing the present model with a different measure of appraisal. Namely, the results may differ if examined with a measure of appraisal demonstrating greater internal consistency. If appraisal is conceptualized consistent with the current framework, researchers wishing to pursue this possibility will need to determine which dimension of appraisal (i.e. motivational relevance, motivational congruence, self-accountability) they would like the measure to reflect, given that the three do not appear to vary together. Once again, the present results would suggest that motivational congruence is most relevant to perfectionism. If appraisal is conceptualized differently (e.g. stable versus unstable, global versus specific, controllable versus uncontrollable; Abramson, Seligman, \& Teasdale, 1978), researchers will need to make a compelling case for why such dimensions would be specifically relevant to perfectionism.

\section{Conclusion}

Socially prescribed perfectionism was found to be associated with greater demoralization even in the absence of a greater number of recent negative life events. Whereas self-oriented perfectionism did not demonstrate a significant, unique relationship to demoralization, it may increase vulnerability for this and other concerning outcomes through a pathway of socially prescribed perfectionism. In considering the cognitive mechanism of perfectionism's effects, appraisals of falling short of standards may be particularly relevant. These present results 
contribute to the broader literature on perfectionism, which has begun to reveal its subtle, but pernicious, consequences. This study serves as a foundation from which more complex research questions may be proposed and, eventually, from which clinical applications may be developed and implemented. 


\section{References}

Abramson, L. Y., Seligman, M. E., \& Teasdale, J. D. (1978). Learned helplessness in humans: Critique and reformulation. Journal of Abnormal Psychology, 87(1), 49.

Adler, A. (1931). What life should mean to you. Oxford, England: Little, Brown.

Aiken, L. S., West, S. G., \& Reno, R. R. (1991). Multiple regression: Testing and interpreting interactions: London: Sage.

Alloy, L. B., Abramson, L. Y., Whitehouse, W. G., Hogan, M. E., Tashman, N. A., Steinberg, D. L., .. . Donovan, P. (1999). Depressogenic cognitive styles: Predictive validity, information processing and personality characteristics, and developmental origins. Behaviour research and therapy, 37(6), 503-531.

Amodeo, D. (2014). Perfectionism and social connectedness in graduate students (Doctoral dissertation). Retreived from ProQuest Dissertations Publishing. (3620430)

Anestis, J. C., Finn, J. A., Gottfried, E. D., Hames, J. L., Bodell, L. P., Hagan, C. R., . . Joiner, T. E. (2018). Burdensomeness, Belongingness, and Capability: Assessing the Interpersonal-Psychological Theory of Suicide With MMPI-2-RF Scales. Assessment, $25(4), 415-431$.

Antony, M. M., Purdon, C. L., Huta, V., \& Swinson, R. P. (1998). Dimensions of perfectionism across the anxiety disorders1. Behaviour research and therapy, 36(12), 1143-1154.

Antonovsky, A. (1979). Health, stress, and coping. San Francisco: Jossey-Bass.

Armeli, S., Conner, T. S., Covault, J., Tennen, H., \& Kranzler, H. R. (2008). A serotonin transporter gene polymorphism (5-HTTLPR), drinking-to-cope motivation, and negative 
life events among college students. Journal of studies on alcohol and drugs, 69(6), 814823.

Arnold, H. J., \& Feldman, D. C. (1981). Social desirability response bias in self-report choice situations. Academy of Management Journal, 24(2), 377-385.

Ash, C., \& Huebner, E. S. (2001). Environmental events and life satisfaction reports of adolescents: A test of cognitive mediation. School Psychology International, 22(3), 320336.

Bagge, C. L., Glenn, C. R., \& Lee, H.-J. (2013). Quantifying the impact of recent negative life events on suicide attempts. Journal of Abnormal Psychology, 122(2), 359.

Bardone-Cone, A. M. (2007). Self-oriented and socially prescribed perfectionism dimensions and their associations with disordered eating. Behaviour research and therapy, 45(8), 1977-1986.

Baron, R. M., \& Kenny, D. A. (1986). The moderator-mediator variable distinction in social psychological research: Conceptual, strategic, and statistical considerations. Journal of personality and social psychology, 51(6), 1173.

Baum, A., Fleming, I., \& Singer, J. E. (1983). Coping with technological disaster. Journal of Social Issues, 39, 117-138.

Baumeister, R. F. (1990). Suicide as escape from self. Psychological review, 97(1), 90.

Beevers, C. G., \& Miller, I. W. (2004). Perfectionism, cognitive bias, and hopelessness as prospective predictors of suicidal ideation. Suicide and life-threatening behavior, 34(2), 126-137. 
Bennett, P., Lowe, R., \& Honey, K. (2003). Brief Report; Appraisals, core relational themes, and emotions: A test of the consistency of reporting and their associations. Cognition and Emotion, 17(3), 511-520.

Bernstein, I. H., \& Nunnally, J. C. (1994). Psychometric theory. New York: McGraw-Hill. Oliva, TA, Oliver, RL, \& MacMillan, IC (1992). A catastrophe model for developing service satisfaction strategies. Journal of Marketing, 56, 83-95

Besser, Flett, \& Hewitt. (2004). Perfectionism, Cognition, and Affect in Response to Performance Failure vs. Success. Journal of Rational - Emotive \& Cognitive - Behavior Therapy, 22(4), 297-324. doi:http://dx.doi.org/10.1023/B:JORE.0000047313.35872.5c

Besser, Flett, Hewitt, \& Guez. (2008). Perfectionism, and Cognitions, Affect, Self-esteem, and Physiological Reactions in a Performance Situation. Journal of Rational - Emotive \& Cognitive - Behavior Therapy, 26(3), 206-228. doi:http://dx.doi.org/10.1007/s10942-007$0067-0$

Bieling, P. J., Israeli, A. L., \& Antony, M. M. (2004). Is perfectionism good, bad, or both? Examining models of the perfectionism construct. Personality and Individual Differences, 36(6), 1373-1385.

Bissell, K., \& Rask, A. (2010). Real women on real beauty: Self-discrepancy, internalisation of the thin ideal, and perceptions of attractiveness and thinness in Dove's Campaign for Real Beauty. International Journal of Advertising, 29(4), 643-668.

Blankstein, K. R., Flett, Hewitt, P. L., \& Eng, A. (1993). Dimensions of perfectionism and irrational fears: An examination with the fear survey schedule. Personality and Individual Differences, 15(3), 323-328. 
Blankstein, K. R., Lumley, C. H., \& Crawford, A. (2007). Perfectionism, hopelessness, and suicide ideation: Revisions to diathesis-stress and specific vulnerability models. Journal of Rational-Emotive \& Cognitive-Behavior Therapy, 25(4), 279-319.

Blatt, S. J. (1995). The destructiveness of perfectionism: Implications for the treatment of depression. American psychologist, 50(12), 1003.

Blatt, S. J., Zuroff, D. C., Quinlan, D. M., \& Pilkonis, P. A. (1996). Interpersonal factors in brief treatment of depression: Further analyses of the National Institute of Mental Health Treatment of Depression Collaborative Research Program. J Consult Clin Psychol, 64(1), 162.

Bollen, K. A. (1984). Multiple indicators: Internal consistency or no necessary relationship?. Quality and Quantity, 18(4), 377-385.

Brocklesby, M. (2017). A longitudinal investigation of non-suicidal self-injury and perfectionism in a sample of New Zealand adolescents (Doctoral dissertation). Retreived from ProQuest Dissertations Publishing.

Brown, A. J., Parman, K. M., Rudat, D. A., \& Craighead, L. W. (2012). Disordered eating, perfectionism, and food rules. Eat Behav, 13(4), 347-353.

Brown, G. (1974). Meaning, measurement, and stress of life events. In B. S. Dohrenwend \& B. P. Dohrenwend (Eds.), Stressful life events: Their nature and effects, New York: John Wiley.

Brüdern, J., Berger, T., Michel, K., Maillart, A. G., Held, I. S., \& Caspar, F. (2015). Are suicide attempters wired differently?: a comparison with nonsuicidal depressed individuals using plan analysis. J Nerv Ment Dis, 203(7), 514-521. 
Buhlmann, U., Etcoff, N. L., \& Wilhelm, S. (2008). Facial attractiveness ratings and perfectionism in body dysmorphic disorder and obsessive-compulsive disorder. Journal of anxiety disorders, 22(3), 540-547.

Bulik, C. M., Carpenter, L. L., Kupfer, D. J., \& Frank, E. (1990). Features associated with suicide attempts in recurrent major depression. J Affect Disord, 18(1), 29-37.

Burns, (1980). The perfectionist's script for self-defeat. Psychology today, 14(6), 34-52.

Burns, V. E., Carroll, D., Drayson, M., Whitham, M., \& Ring, C. (2003). Life events, perceived stress and antibody response to influenza vaccination in young, healthy adults. Journal of Psychosomatic Research, 55(6), 569-572.

Burns, V. E., Drayson, M., Ring, C., \& Carroll, D. (2002). Perceived stress and psychological well-being are associated with antibody status after meningitis $\mathrm{C}$ conjugate vaccination. Psychosomatic Medicine, 64(6), 963-970.

Cameron, A. C. (2004). Kurtosis. In M. Lewis-Beck, A. E. Bryman, \& T. F. Liao (Eds.), The Sage Encyclopedia of Social Science Research Methods (Vol. 2, pp. 543-544): Sage Publications.

Campos, Holden, R. R., Caçador, B., Fragata, A. S., \& Baleizão, C. (2018). Self-criticism, intensity of perceived negative life events, and distress: Results from a two-wave study. Personality and Individual Differences, 124, 145-149.

Caspar, F. (2007). Beziehungen und Probleme verstehen: Eine Einführung in die psychotherapeutische Plan Analyse [Understanding relationships and problems: An introduction to the psychotherapeutic plan analysis]. Bern, Switzerland: Huber. 
Catanese, A. A., John, M. S., Di Battista, J., \& Clarke, D. M. (2009). Acute cognitive therapy in reducing suicide risk following a presentation to an emergency department. Behaviour Change, 26(1), 16-26.

Chang, E. C., \& Rand, K. L. (2000). Perfectionism as a predictor of subsequent adjustment: Evidence for a specific diathesis-stress mechanism among college students. Journal of Counseling Psychology, 47(1), 129.

Chang, E. C., \& Sanna, L. J. (2001). Negative attributional style as a moderator of the link between perfectionism and depressive symptoms: Preliminary evidence for an integrative model. Journal of Counseling Psychology, 48(4), 490.

Chang, E. C., Sanna, L. J., Chang, R., \& Bodem, M. R. (2008). A preliminary look at loneliness as a moderator of the link between perfectionism and depressive and anxious symptoms in college students: Does being lonely make perfectionistic strivings more distressing?. Behaviour Research and Therapy, 46(7), 877-886.

Chatard, A., \& Selimbegović, L. (2011). When self-destructive thoughts flash through the mind: Failure to meet standards affects the accessibility of suicide-related thoughts. Journal of personality and social psychology, 100(4), 587.

Chen, C., Hewitt, P. L., \& Flett, (2015). Preoccupied attachment, need to belong, shame, and interpersonal perfectionism: An investigation of the perfectionism social disconnection model. Personality and Individual Differences, 76, 177-182.

Cheng, S. K. (2001). Life stress, problem solving, perfectionism, and depressive symptoms in Chinese. Cognitive therapy and research, 25(3), 303-310. 
Childs, J. H., \& Stoeber, J. (2010). Self-oriented, other-oriented, and socially prescribed perfectionism in employees: Relationships with burnout and engagement. Journal of Workplace Behavioral Health, 25(4), 269-281.

Claes, L., Soenens, B., Vansteenkiste, M., \& Vandereycken, W. (2012). The Scars of the Inner Critic: Perfectionism and Nonsuicidal Self-Injury in Eating Disorders. European Eating Disorders Review, 20(3), 196-202.

Clarke, D. M., \& Kissane, D. W. (2002). Demoralization: its phenomenology and importance. Australian and New Zealand Journal of Psychiatry, 36(6), 733-742.

Clements, K., \& Turpin, G. (1996). The life events scale for students: validation for use with British samples. Personality and Individual Differences, 20(6), 747-751.

Cohen, L. H., Gunthert, K. C., Butler, A. C., O’Neill, S. C., \& Tolpin, L. H. (2005). Daily affective reactivity as a prospective predictor of depressive symptoms. Journal of Personality, 73, 1-27.

Conroy, D. E., Kaye, M. P., \& Fifer, A. M. (2007). Cognitive links between fear of failure and perfectionism. Journal of Rational-Emotive \& Cognitive-Behavior Therapy, 25(4), 237253.

Conway, C. C., Hammen, C., Espejo, E. P., Wray, N. R., Najman, J. M., \& Brennan, P. A. (2012). Appraisals of stressful life events as a genetically-linked mechanism in the stress-depression relationship. Cognitive Therapy and Research, 36(4), 338-347.

Coo, S., Milgrom, J., Kuppens, P., \& Trinder, J. (2015). Perinatal distress, an appraisal perspective. Journal of Reproductive and Infant Psychology, 33(2), 190-204.

Corrigan, P. W., \& Watson, A. C. (2002). The paradox of self-stigma and mental illness. Clinical Psychology: Science and Practice, 9(1), 35-53. 
Covault, J., Tennen, H., Armeli, S., Conner, T. S., Herman, A. I., Cillessen, A. H., \& Kranzler, H. R. (2007). Interactive effects of the serotonin transporter 5-HTTLPR polymorphism and stressful life events on college student drinking and drug use. Biol Psychiatry, 61(5), 609-616.

Curran, T., \& Hill, A. P. (2017). Perfectionism Is Increasing Over Time: A Meta-Analysis of Birth Cohort Differences From 1989 to 2016. Psychological Bulletin.

De Jong, C. A., Kissane, D. W., Geessink, R. J., \& Velden, D. (2008). Demoralization in opioid dependent patients: a comparative study with cancer patients and community subjects. The Open Addiction Journal 1, 7-9.

Dean, \& Range. (1996). The escape theory of suicide and perfectionism in college students. Death studies, 20(4), 415-424.

Dean, \& Range. (1999). Testing the escape theory of suicide in an outpatient clinical population. Cognitive therapy and research, 23(6), 561-572.

Dean, Range, \& Goggin. (1996). The escape theory of suicide in college students: Testing a model that includes perfectionism. Suicide and life-threatening behavior, 26(2), 181-186.

Domino, G. (2005). Cross-cultural attitudes towards suicide: the SOQ and a personal odyssey. Archives of Suicide Research, 9(2), 107-122.

Downey, C. A., \& Chang, E. C. (2007). Perfectionism and symptoms of eating disturbances in female college students: Considering the role of negative affect and body dissatisfaction. Eat Behav, 8(4), 497-503.

Dunkley, D. M., \& Blankstein, K. R. (2000). Self-critical perfectionism, coping, hassles, and current distress: A structural equation modeling approach. Cognitive therapy and research, 24(6), 713-730. 
Egan, S. J., Hattaway, M., \& Kane, R. T. (2014). The relationship between perfectionism and rumination in post traumatic stress disorder. Behavioural and cognitive psychotherapy, 42(2), 211-223.

Enns, M. W., \& Cox, B. J. (1999). Perfectionism and depression symptom severity in major depressive disorder. Behaviour research and therapy, 37(8), 783-794.

Enns, M. W., \& Cox, B. J. (2005). Perfectionism, stressful life events, and the 1-year outcome of depression. Cognitive therapy and research, 29(5), 541-553.

Enns, M. W., Cox, B. J., Sareen, J., \& Freeman, P. (2001). Adaptive and maladaptive perfectionism in medical students: a longitudinal investigation. Medical education, $35(11), 1034-1042$.

Evans, G. W., Jacobs, S. V., Dooley, D., \& Catalano, R. (1987). The interaction of stressful life events and chronic strains on community mental health. American journal of community psychology, 15(1), 23-34.

Fang, C.-K., Chang, M.-C., Chen, P.-J., Lin, C.-C., Chen, G.-S., Lin, J., . . W Wu, C.-L. (2014). A correlational study of suicidal ideation with psychological distress, depression, and demoralization in patients with cancer. Supportive Care in Cancer, 22(12), 3165-3174.

Flamenbaum, R., \& Holden, R. R. (2007). Psychache as a mediator in the relationship between perfectionism and suicidality. Journal of Counseling Psychology, 54(1), 51.

Flett, Greene, A., \& Hewitt, (2004). Dimensions of perfectionism and anxiety sensitivity. Journal of Rational-Emotive and Cognitive-Behavior Therapy, 22(1), 39-57.

Flett, \& Hewitt, (2014). A proposed framework for preventing perfectionism and promoting resilience and mental health among vulnerable children and adolescents. Psychology in the Schools, 51(9), 899-912. 
Flett, Hewitt, P. L., Blankstein, K., \& O'Brien, S. (1991). Perfectionism and learned resourcefulness in depression and self-esteem. Personality and Individual Differences, 12(1), 61-68.

Flett, Hewitt, P. L., Blankstein, K. R., \& Mosher, S. W. (1995). Perfectionism, life events, and depressive symptoms: A test of a diathesis-stress model. Current Psychology, 14(2), 112137.

Flett, Hewitt, P. L., Blankstein, K. R., Solnik, M., \& Van Brunschot, M. (1996). Perfectionism, social problem-solving ability, and psychological distress. Journal of Rational-Emotive and Cognitive-Behavior Therapy, 14(4), 245-274.

Flett, Hewitt, P. L., \& De Rosa, T. (1996). Dimensions of perfectionism, psychosocial adjustment, and social skills. Personality and Individual Differences, 20(2), 143-150.

Flett, Hewitt, P. L., Garshowitz, M., \& Martin, T. R. (1997). Personality, negative social interactions, and depressive symptoms. Canadian Journal of Behavioural Science/Revue canadienne des sciences du comportement, 29(1), 28.

Foa, E. B., Ehlers, A., Clark, D. M., Tolin, D. F., \& Orsillo, S. M. (1999). The posttraumatic cognitions inventory (PTCI): Development and validation. Psychological Assessment, 11(3), 303.

Folkman, S., \& Lazarus, R. S. (1980). An analysis of coping in a middleaged community sample. Journal of Health and Social Behavior, 21, 219-239.

Folkman, S., \& Lazarus, R. S. (1985). If it changes it must be a process: A study of emotion and coping during three stages of a college examination. Journal of Personality and Social Psychology, 48,150-170. 
Folkman, S., Lazarus, R. S., Dunkel-Schetter, C., DeLongis, A., \& Gruen, R. J. (1986).

Dynamics of a stressful encounter: Cognitive appraisal, coping, and encounter outcomes. Journal of Personality and Social Psychology, 50, 992-1003.

Folkman, S., Lazarus, R. S., Gruen, R. J., \& DeLongis, A. (1986). Appraisal, coping, health status, and psychological symptoms. Journal of personality and social psychology, 50(3), 571.

Frank, J. D. (1974). Psychotherapy: The restoration of morale. American Journal of Psychiatry, 131(3), 271-274.

Frank, J. D., \& Frank, J. B. (1993). Persuasion and healing: A comparative study of psychotherapy: London: JHU Press.

Frost, R. O., Heimberg, R. G., Holt, C. S., Mattia, J. I., \& Neubauer, A. L. (1993). A comparison of two measures of perfectionism. Personality and Individual Differences, 14(1), 119126.

Frost, R. O., \& Henderson, K. J. (1991). Perfectionism and reactions to athletic competition. Journal of Sport and Exercise Psychology, 13(4), 323-335.

Frost, R. O., Marten, P., Lahart, C., \& Rosenblate, R. (1990). The dimensions of perfectionism. Cognitive therapy and research, 14(5), 449-468.

Frost, R. O., \& Steketee, G. (1997). Perfectionism in obsessive-compulsive disorder patients. Behaviour research and therapy, 35(4), 291-296.

Fry, P. S., \& Debats, D. L. (2009). Perfectionism and the five-factor personality traits as predictors of mortality in older adults. Journal of Health Psychology, 14(4), 513-524.

Gilbert, P., \& Allan, S. (1998). The role of defeat and entrapment (arrested flight) in depression: an exploration of an evolutionary view. Psychol Med, 28(3), 585-598. 
Gould, M. S., Fisher, P., Parides, M., Flory, M., \& Shaffer, D. (1996). Psychosocial risk factors of child and adolescent completed suicide. Arch Gen Psychiatry, 53(12), 1155-1162.

Grawe, K. (1980). Die diagnostisch-therapeutische Funktion der Gruppeninteraktion in verhaltenstherapeutischen Gruppen. Verhaltenstherapie in gruppen, 88-223.

Griffith, J. L., \& Gaby, L. (2005). Brief psychotherapy at the bedside: countering demoralization from medical illness. Psychosomatics, 46(2), 109-116.

Griffiths, A. W., Wood, A. M., Maltby, J., Taylor, P. J., \& Tai, S. (2014). The prospective role of defeat and entrapment in depression and anxiety: A 12-month longitudinal study. Psychiatry Res, 216(1), 52-59.

Gross. (1998). Antecedent-and response-focused emotion regulation: divergent consequences for experience, expression, and physiology. Journal of personality and social psychology, 74(1), 224.

Gross, J., Rosen, J. C., Leitenberg, H., \& Willmuth, M. E. (1986). Validity of the Eating Attitudes Test and the Eating Disorders Inventory in bulimia nervosa. J Consult Clin Psychol, 54(6), 875.

Grzegorek, J. L., Slaney, R. B., Franze, S., \& Rice, K. G. (2004). Self-Criticism, Dependency, Self-Esteem, and Grade Point Average Satisfaction Among Clusters of Perfectionists and Nonperfectionists. Journal of Counseling Psychology, 51(2), 192.

Hadnagy, Z., Csikós, Á., \& Nagy, L. (2012). Introduction of the demoralization scale in Hungary, a study on demoralization and depression among hospice patients. Orvosi hetilap, 153(19), 737-743. 
Haine, R. A., Ayers, T. S., Sandler, I. N., Wolchik, S. A., \& Weyer, J. L. (2003). Locus of control and self-esteem as stress-moderators or stress-mediators in parentally bereaved children. Death studies, 27(7), 619-640.

Hair, J. F. Jr., Black, W. C., Babin, B. J., Anderson, R. E. (2010). Multivariate Data Analysis (7 $7^{\text {th }}$ ed). New York: Macmillan.

Hallaraker, E., Arefjord, K., Havik, O. E., \& Mæland, J. G. (2001). Social support and emotional adjustment during and after a severe life event: A study of wives of myocardial infarction patients. Psychology and Health, 16(3), 343-355.

Hamachek, D. E. (1978). Psychodynamics of normal and neurotic perfectionism. Psychology: A Journal of Human Behavior, 15(1), 27-33.

Hamilton, T. K., \& Schweitzer, R. D. (2000). The cost of being perfect: Perfectionism and suicide ideation in university students. Australian \& New Zealand Journal of Psychiatry, 34(5), 829-835.

Handley, A. K., Egan, S. J., Kane, R. T., \& Rees, C. S. (2014). The relationships between perfectionism, pathological worry and generalised anxiety disorder. BMC Psychiatry, 14(1), 98 .

Hankin, B. L., Roberts, J., \& Gotlib, I. H. (1997). Elevated self-standards and emotional distress during adolescence: Emotional specificity and gender differences. Cognitive therapy and research, 21(6), 663-679.

Hayes, A. F. (2013). Introduction to mediation, moderation, and conditional process analysis: A regression-based approach. New York, NY: Guilford Press.

Hayes, A. F. (2019). FAQ- The PROCESS macro for SPSS and SAS. Retireved from http://processmacro.org/faq.html 
Heidrich, S. (1994). The self, health, and depression in elderly women. Western Journal of Nursing Research, 16(5), 544-555.

Heidrich, S. (1999). Self-discrepancy across the life span. Journal of Adult Development, 6(2), $119-130$.

Henrich, J., Heine, S. J., \& Norenzayan, A. (2010). Beyond WEIRD: Towards a broad-based behavioral science. Behavioral and Brain Sciences, 33(2-3), 111.

Herman, K. C., Trotter, R., Reinke, W. M., \& Ialongo, N. (2011). Developmental origins of perfectionism among African American youth. J Couns Psychol, 58(3), 321-334. doi:10.1037/a0023108

Hewitt, P. L., Caelian, C. F., Chen, C., \& Flett. (2014). Perfectionism, stress, daily hassles, hopelessness, and suicide potential in depressed psychiatric adolescents. Journal of Psychopathology and Behavioral Assessment, 36(4), 663-674.

Hewitt, Caelian, Flett, Sherry, Collins, \& Flynn. (2002). Perfectionism in children: Associations with depression, anxiety, and anger. Personality and Individual Differences, 32(6), 10491061.

Hewitt, P. L., \& Dyck, D. G. (1986). Perfectionism, stress, and vulnerability to depression. Cognitive therapy and research, 10(1), 137-142.

Hewitt, \& Flett. (1989). The multidimensional perfectionism scale: Development and validation. Canadian Psychology, 30(1), 339.

Hewitt, \& Flett. (1990). Perfectionism and depression: A multidimensional analysis. Journal of Social Behavior and Personality, 5(5), 423.

Hewitt, \& Flett. (1991a). Dimensions of perfectionism in unipolar depression. Journal of Abnormal Psychology, 100(1), 98. 
Hewitt, \& Flett. (1991b). Perfectionism in the self and social contexts: conceptualization, assessment, and association with psychopathology. Journal of personality and social psychology, 60(3), 456.

Hewitt, \& Flett. (1993). Dimensions of perfectionism, daily stress, and depression: a test of the specific vulnerability hypothesis. Journal of Abnormal Psychology, 102(1), 58.

Hewitt, Flett, \& Ediger. (1996). Perfectionism and depression: Longitudinal assessment of a specific vulnerability hypothesis. Journal of Abnormal Psychology, 105(2), 276.

Hewitt, Flett, Sherry, \& Caelian. (2006). Trait Perfectionism Dimensions and Suicidal Behavior. In T. E. Ellis (Ed.), Cognition and suicide: Theory, research, and therapy (pp. 215-235). Washington, D.C.: American Psychological Association.

Hewitt, P. L., Flett, \& Ediger, E. (1995). Perfectionism traits and perfectionistic self-presentation in eating disorder attitudes, characteristics, and symptoms. International Journal of Eating Disorders, 18(4), 317-326.

Hewitt, P. L., Flett, Turnbull-Donovan, W., \& Mikail, S. F. (1991). The Multidimensional Perfectionism Scale: Reliability, validity, and psychometric properties in psychiatric samples. Psychological Assessment: A Journal of Consulting and Clinical Psychology, 3(3), 464.

Hewitt, P. L., Flett, \& Turnbull-Donovan, W. (1992). Perfectionism and suicide potential. British Journal of Clinical Psychology, 31(2), 181-190.

Hewitt, P. L., Flett, \& Turnbull, W. (1992). Perfectionism and multiphasic personality inventory (MMPI) indices of personality disorder. Journal of Psychopathology and Behavioral Assessment, 14(4), 323-335. 
Hewitt, P. L., Flett, \& Weber, C. (1994). Dimensions of perfectionism and suicide ideation. Cognitive therapy and research, 18(5), 439-460.

Hewitt, P. L., Norton, G. R., Flett, Callander, L., \& Cowan, T. (1998). Dimensions of perfectionism, hopelessness, and attempted suicide in a sample of alcoholics. Suicide and life-threatening behavior, 28(4), 395-406.

Hill, Hall, Duda, \& Appleton. (2011). The cognitive, affective and behavioural responses of selforiented perfectionists following successive failure on a muscular endurance task. International Journal of Sport and Exercise Psychology, 9(2), 189-207.

Hill, Zrull, \& Turlington. (1997). Perfectionism and interpersonal problems. Journal of Personality Assessment, 69(1), 81-103.

Hirsch, J. K., Wolford, K., LaLonde, S. M., Brunk, L., \& Parker-Morris, A. (2009). Optimistic explanatory style as a moderator of the association between negative life events and suicide ideation. Crisis: The Journal of Crisis Intervention and Suicide Prevention, 30(1), 48.

Hoff, E. R., \& Muehlenkamp, J. J. (2009). Nonsuicidal self-injury in college students: The role of perfectionism and rumination. Suicide and life-threatening behavior, 39(6), 576-587.

Hollender, M. H. (1965). Perfectionism. Compr Psychiatry, 6(2), 94-103.

Holmbeck, G. N. (1997). Toward terminological, conceptual, and statistical clarity in the study of mediators and moderators: Examples from the child-clinical and pediatric psychology literatures. J Consult Clin Psychol, 65(4), 599.

Holmbeck, G. N. (2002). Post-hoc probing of significant moderational and mediational effects in studies of pediatric populations. Journal of pediatric psychology, 27(1), 87-96. 
Holmes, T. H., \& Rahe, R. H. (1967). The social readjustment rating scale. Journal of Psychosomatic Research, 11(2), 213-218.

Holt, L., Armeli, S., Tennen, H., Austad, C. S., Raskin, S., Fallahi, C., . . Pearlson, G. D. (2013). A person-centered approach to understanding negative reinforcement drinking among first year college students. Addictive Behaviors, 38(12), 2937-2944.

Hooley, J. M., \& Teasdale, J. D. (1989). Predictors of relapse in unipolar depressives: Expressed emotion, marital distress, and perceived criticism. Journal of Abnormal Psychology, 98(3), 229.

Hung, H., Chen, H., Chang, Y., Yang, Y., Liu, C., Hsieh, R., . . Tsai, L. (2010). Evaluation of the reliability and validity of the Mandarin Version of Demoralization Scale for cancer patients. J Intern Med Taiwan, 21(6), 427-435.

Hunter, E. C., \& O'Connor (2003). Hopelessness and future thinking in parasuicide: The role of perfectionism. British Journal of Clinical Psychology, 42(4), 355-365.

Iketani, T., Kiriike, N., Stein, M. B., Nagao, K., Nagata, T., Minamikawa, N., . . Fukuhara, H. (2002). Relationship between perfectionism and agoraphobia in patients with panic disorder. Cognitive Behaviour Therapy, 31(3), 119-128.

Jackson, Y., Kim, K. L., \& Delap, C. (2007). Mediators of control beliefs, stressful life events, and adaptive behavior in school age children: The role of appraisal and social support. Journal of traumatic stress, 20(2), 147-160.

Jacobs, R. H., Silva, S. G., Reinecke, M. A., Curry, J. F., Ginsburg, G. S., Kratochvil, C. J., \& March, J. S. (2009). Dysfunctional attitudes scale perfectionism: a predictor and partial mediator of acute treatment outcome among clinically depressed adolescents. Journal of Clinical Child \& Adolescent Psychology, 38(6), 803-813. 
Jacobsen, J. C., Vanderwerker, L. C., Block, S. D., Friedlander, R. J., Maciejewski, P. K., \& Prigerson, H. G. (2006). Depression and demoralization as distinct syndromes: Preliminary data from a cohort of advanced cancer patients. Indian Journal of Palliative Care, 12(1), 8 .

Janoff-Bulman, R., \& Morgan, H. J. (1994). Victims' Responses to traumatic life events: An unjust world or an uncaring world? Social Justice Research, 7(1), 47-68.

Johnson, J., Gooding, P. A., Wood, A. M., \& Tarrier, N. (2010). Resilience as positive coping appraisals: Testing the schematic appraisals model of suicide (SAMS). Behaviour research and therapy, 48(3), 179-186.

Johnson, P. O., \& Fay, L. C. (1950). The Johnson-Neyman technique, its theory and application. Psychometrika, 15(4), 349-367.

Johnson, P. O., \& Neyman, J. (1936). Tests of certain linear hypotheses and their application to some educational problems. Statistical research memoirs 1, 57-93.

Joiner, T. E. (2005). Why people die by suicide. Cambridge, MA: Harvard University Press.

Joiner Jr, T. E., \& Rudd, M. D. (2000). Intensity and duration of suicidal crises vary as a function of previous suicide attempts and negative life events. J Consult Clin Psychol, 68(5), 909.

Joiner, T. E., \& Schmidt, N. B. (1995). Dimensions of perfectionism, life stress, and depressed and anxious symptoms: Prospective support for diathesis-stress but not specific vulnerability among male undergraduates. Journal of Social and Clinical Psychology, 14(2), 165-183.

Juster, H. R., Heimberg, R. G., Frost, R. O., Holt, C. S., Mattia, J. I., \& Faccenda, K. (1996). Social phobia and perfectionism. Personality and Individual Differences, 21(3), 403-410. 
Kaplan, M. L., Asnis, G. M., Sanderson, W. C., Keswani, L., de Lecuona, J. M., \& Joseph, S. (1994). Suicide assessment: Clinical interview vs. self-report. Journal of Clinical Psychology, 50(2), 294-298.

Kissane, D. W. (2004). The contribution of demoralization to end of life decisionmaking. Hastings Center Report, 34(4), 21-31.

Kissane, D. W., Clarke, D. M., \& Street, A. F. (2001). Demoralization syndrome--a relevant psychiatric diagnosis for palliative care. Journal of palliative care, 17(1), 12.

Kissane, D. W., Levin, T., Hales, S., Lo, C., \& Rodin, G. (2011). Psychotherapy for depression in cancer and palliative care. Depression and Cancer. Chichester: Wiley, 177-206.

Kissane, D. W., Wein, S., Love, A., \& Lee, X. Q. (2004). The Demoralization Scale: a report of its development and preliminary validation. Journal of palliative care, 20(4), 269.

Klibert, J. J., Langhinrichsen-Rohling, J., \& Saito, M. (2005). Adaptive and maladaptive aspects of self-oriented versus socially prescribed perfectionism. Journal of College Student Development, 46(2), 141-156.

Knafo, A., \& Jaffee, S. R. (2013). Gene-environment correlation in developmental psychopathology. Development and psychopathology, 25(1), 1-6.

Kobori, O., \& Tanno, Y. (2005). Self-oriented perfectionism and its relationship to positive and negative affect: The mediation of positive and negative perfectionism cognitions. Cognitive therapy and research, 29(5), 555-567.

Kowal, A., \& Pritchard, D. (1990). Psychological characteristics of children who suffer from headache: a research note. $J$ Child Psychol Psychiatry, 31(4), 637-649.

Kranzler, H. R., Scott, D., Tennen, H., Feinn, R., Williams, C., Armeli, S., . . Covault, J. (2012). The 5-HTTLPR polymorphism moderates the effect of stressful life events on 
drinking behavior in college students of African descent. American Journal of Medical Genetics Part B: Neuropsychiatric Genetics, 159(5), 484-490.

Lance, C. E., Dawson, B., Birkelbach, D., \& Hoffman, B. J. (2010). Method effects, measurement error, and substantive conclusions. Organizational Research Methods, $13(3), 435-455$.

Lantz, P. M., House, J. S., Mero, R. P., \& Williams, D. R. (2005). Stress, life events, and socioeconomic disparities in health: results from the Americans' Changing Lives Study. Journal of Health and Social Behavior, 46(3), 274-288.

Lau, R., Morse, C. A., \& Macfarlane, S. (2010). Psychological factors among elderly women with suicidal intentions or attempts to suicide: a controlled comparison. Journal of women \& aging, 22(1), 3-14.

Lazarus, R. S. (1966). Psychological stress and the coping process. New York, NY: McGrawHill.

Lazarus, R. S., \& Folkman, S. (1984). The handbook of behavioral medicine. New York, NY: Guilford Press.

Lee, C.-Y., Fang, C.-K., Yang, Y.-C., Liu, C.-L., Leu, Y.-S., Wang, T.-E., . . Tsai, L.-Y. (2012). Demoralization syndrome among cancer outpatients in Taiwan. Supportive Care in Cancer, 20(10), 2259-2267.

Lee-Flynn, S. C., Pomaki, G., DeLongis, A., Biesanz, J. C., \& Puterman, E. (2011). Daily cognitive appraisals, daily affect, and long-term depressive symptoms: The role of selfesteem and self-concept clarity in the stress process. Personality and Social Psychology Bulletin, 37(2), 255-268. 
Lee, I. A., \& Preacher, K. J. (2013, September). Calculation for the test of the difference between two dependent correlations with one variable in common [Computer software]. Available from http://quantpsy.org.

Lenhard, W. \& Lenhard, A. (2016). Calculation of Effect Sizes. Retrieved from: https://www.psychometrica.de/effect_size.html. Dettelbach (Germany): Psychometrica. DOI: $10.13140 /$ RG.2.1.3478.4245

Lennon, M. C., Dohrenwend, B. P., Zautra, A. J., \& Marbach, J. J. (1990). Coping and adaptation to facial pain in contrast to other stressful life events. Journal of personality and social psychology, 59(5), 1040.

Limburg, K., Watson, H. J., Hagger, M. S., \& Egan, S. J. (2017). The relationship between perfectionism and psychopathology: A meta-analysis. J Clin Psychol, 73(10), 1301-1326.

Linden, W. (1984). Development and initial validation of a life event scale for students. Canadian Journal of Counselling and Psychotherapy/Revue canadienne de counseling et de psychothérapie, 18(3).

Liu, Liu, L., Yang, J., \& Zhao, G. (1997). Reliability and validity of the adolescents self-rating life events checklist. Chinese Journal of Clinical Psychology, 5(1), 34-36.

Liu, X., \& Tein, J.-Y. (2005). Life events, psychopathology, and suicidal behavior in Chinese adolescents. J Affect Disord, 86(2), 195-203.

Lowe, R., \& Bennett, P. (2003). Exploring coping reactions to work-stress: Application of an appraisal theory. Journal of Occupational and Organizational Psychology, 76(3), 393400.

Lowe, R., Vedhara, K., Bennett, P., Brookes, E., Gale, L., Munnoch, K., ... \& Farndon, J. (2003). Emotion-related primary and secondary appraisals, adjustment and coping: Associations 
in women awaiting breast disease diagnosis. British journal of health psychology, 8(4), 377-391.

Lynd-Stevenson, R. M., \& Hearne, C. M. (1999). Perfectionism and depressive affect: the pros and cons of being a perfectionist. Personality and Individual Differences, 26(3), 549-562.

Madge, N., Hawton, K., McMahon, E. M., Corcoran, P., De Leo, D., De Wilde, E. J., . . . Arensman, E. (2011). Psychological characteristics, stressful life events and deliberate self-harm: findings from the Child \& Adolescent Self-harm in Europe (CASE) Study. European child \& adolescent psychiatry, 20(10), 499.

Manassis, K., Tannock, R., \& Monga, S. (2009). Anxious by maternal-versus self-report: Are they the same children? Journal of the Canadian Academy of Child and Adolescent Psychiatry, 18(2), 103.

Marsa, R., Bahmani, B., Naghiyaee, M., \& Barekati, S. (2017). The Effectiveness of CognitiveExistential Group Therapy on Reducing Demoralization in the Elderly. Middle East Journal of Family Medicine, 7(10), 42.

Martin, T. R., Flett, Hewitt, P. L., Krames, L., \& Szanto, G. (1996). Personality correlates of depression and health symptoms: A test of a self-regulation model. Journal of Research in Personality, 30(2), 264-277.

McFarland, C., \& Alvaro, C. (2000). The impact of motivation on temporal comparisons: Coping with traumatic events by perceiving personal growth. Journal of personality and social psychology, 79(3), 327.

Mehnert, A., Vehling, S., Höcker, A., Lehmann, C., \& Koch, U. (2011). Demoralization and depression in patients with advanced cancer: validation of the German version of the demoralization scale. Journal of pain and symptom management, 42(5), 768-776. 
Mills, J. S., \& Blankstein, K. R. (2000). Perfectionism, intrinsic vs extrinsic motivation, and motivated strategies for learning: A multidimensional analysis of university students. Personality and Individual Differences, 29(6), 1191-1204.

Miskey, H. M., Hill, R. W., \& Huelsman, T. J. (2012). Big five traits and perfectionism are risk factors for nonsuicidal cutting. Psychology and Behavioral Sciences, 1(1), 1-7.

Mitchelson, J. K. (2009). Seeking the perfect balance: Perfectionism and work-family conflict. Journal of Occupational and Organizational Psychology, 82(2), 349-367.

Mullane, M., Dooley, B., Tiernan, E., \& Bates, U. (2009). Validation of the Demoralization Scale in an Irish advanced cancer sample. Palliative \& supportive care, 7(3), 323-330.

Mushquash, A. R., \& Sherry, S. B. (2012). Understanding the socially prescribed perfectionist's cycle of self-defeat: A 7-day, 14-occasion daily diary study. Journal of Research in Personality, 46(6), 700-709.

Myers, J. K., Lindenthal, J. J., Pepper, M. P., \& Ostrander, D. R. (1972). Life events and mental status: A longitudinal study. Journal of Health and Social Behavior, 398-406.

Neumeister, K. L. S. (2004). Understanding the relationship between perfectionism and achievement motivation in gifted college students. Gifted child quarterly, 48(3), 219-231.

Neumeister, K. L. S., \& Finch, H. (2006). Perfectionism in high-ability students: Relational precursors and influences on achievement motivation. Gifted child quarterly, 50(3), 238251.

Newsom, J. T., Rook, K. S., Nishishiba, M., Sorkin, D. H., \& Mahan, T. L. (2005). Understanding the relative importance of positive and negative social exchanges: Examining specific domains and appraisals. The Journals of Gerontology Series B: Psychological Sciences and Social Sciences, 60(6), P304-P312. 
Nikolova, Y. S., Bogdan, R., Brigidi, B. D., \& Hariri, A. R. (2012). Ventral striatum reactivity to reward and recent life stress interact to predict positive affect. Biol Psychiatry, 72(2), 157-163.

O'Connor, (2003). Suicidal behavior as a cry of pain: Test of a psychological model. Archives of Suicide Research, 7(4), 297-308.

O'Connor, (2011). Towards an integrated motivational-volitional model of suicidal behaviour. International handbook of suicide prevention: Research, policy and practice, 1, 181-198.

O'Connor, Rasmussen, S., \& Hawton, K. (2010). Predicting depression, anxiety and self-harm in adolescents: The role of perfectionism and acute life stress. Behaviour research and therapy, 48(1), 52-59.

O'Connor, Rasmussen, S., \& Hawton, K. (2012). Distinguishing adolescents who think about self-harm from those who engage in self-harm. The British Journal of Psychiatry, 200(4), $330-335$.

O'Connor, \& Sheehy, (2000). Understanding suicidal behaviour. Presentation, Stirling, Scotland.

Olson, M. L., \& Kwon, P. (2008). Brooding perfectionism: Refining the roles of rumination and perfectionism in the etiology of depression. Cognitive therapy and research, 32(6), 788802.

Onwuegbuzie, A. J. (2000). Academic procrastinators and perfectionistic tendencies among graduate students. Journal of Social Behavior and Personality, 15(5; SPI), 103-110.

Osvath, P., Vörös, V., \& Fekete, S. (2004). Life events and psychopathology in a group of suicide attempters. Psychopathology, 37(1), 36-40.

Pacht, A. R. (1984). Reflections on perfection. American psychologist, 39(4), 386. 
Panagioti, M., Gooding, P. A., \& Tarrier, N. (2015). A prospective study of suicidal ideation in posttraumatic stress disorder: the role of perceptions of defeat and entrapment. J Clin Psychol, 71(1), 50-61.

Paykel, E. S. (1974). Life stress and psychiatric disorder: Applications of the clinical approach. In B. S. Dohrenwend \& B. P. Dohrenwend (Eds.), Stressful life events: Their nature and effects. Oxford, England: John Wiley \& Sons.

Phillips, A. C., Burns, V. E., Carroll, D., Ring, C., \& Drayson, M. (2005). The association between life events, social support, and antibody status following thymus-dependent and thymus-independent vaccinations in healthy young adults. Brain, behavior, and immunity, 19(4), 325-333.

Portešová, Š., \& Urbánek, T. (2013). Typology of Perfectionism in a Group of Mathematically Gifted Czech Adolescents over One Decade. Journal of Early Adolescence, 33(8), 11161144.

Potthoff, R. F. (1964). On the Johnson-Neyman technique and some extensions thereof. Psychometrika, 29(3), 241-256.

Preacher, K. J., \& Hayes, A. F. (2004). SPSS and SAS procedures for estimating indirect effects in simple mediation models. Behavior Research Methods, Instruments, \& Computers, $36(4), 717-731$.

Rafanelli, C., Guidi, J., Gostoli, S., Tomba, E., Porcelli, P., \& Grandi, S. (2013). Subtyping demoralization in the medically ill by cluster analysis. The European Journal of Psychiatry, 27(1), 7-17.

Rahe, R. H., \& Arthur, R. J. (1978). Life change and illness studies: Past history and future directions. Journal of human stress, 4(1), 3-15. 
Rahe, R. H., \& Lind, E. (1971). Psychosocial factors and sudden cardiac death: A pilot study. Journal of Psychosomatic Research, 15(1), 19-24.

Rasmussen, S. A., Fraser, L., Gotz, M., MacHale, S., Mackie, R., Masterton, G., . . O'connor, (2010). Elaborating the cry of pain model of suicidality: Testing a psychological model in a sample of first-time and repeat self-harm patients. British Journal of Clinical Psychology, 49(1), 15-30.

Rasmussen, S. A., O’Connor, \& Brodie, D. (2008). The role of perfectionism and autobiographical memory in a sample of parasuicide patients: an exploratory study. Crisis, 29(2), 64-72.

Rice, K. G., Ashby, J. S., \& Gilman, R. (2011). Classifying adolescent perfectionists. Psychol Assess, 23(3), 563-577. doi:10.1037/a0022482

Rice, K. G., Ashby, J. S., \& Slaney, R. B. (2007). Perfectionism and the five-factor model of personality. Assessment, 14(4), 385-398.

Rice, K. G., Richardson, C. M., \& Ray, M. E. (2016). Perfectionism in academic settings. In Perfectionism, health, and well-being (pp. 245-264). Springer, Cham.

Richardson, A. S., Bergen, H. A., Martin, G., Roeger, L., \& Allison, S. (2005). Perceived academic performance as an indicator of risk of attempted suicide in young adolescents. Archives of Suicide Research, 9(2), 163-176.

Roxborough, H. M., Hewitt, P. L., Kaldas, J., Flett, Caelian, C. M., Sherry, S., \& Sherry, D. L. (2012). Perfectionistic self-presentation, socially prescribed perfectionism, and Suicide in Youth: A Test of the Perfectionism Social Disconnection Model. Suicide and lifethreatening behavior, 42(2), 217-233. 
Saboonchi, F., Lundh, L.-G., \& Öst, L.-G. (1999). Perfectionism and self-consciousness in social phobia and panic disorder with agoraphobia. Behaviour research and therapy, 37(9), 799-808.

Saddler, C. D., \& Sacks, L. A. (1993). Multidimensional perfectionism and academic procrastination: Relationships with depression in university students. Psychological reports.

Sarason, I. G., Johnson, J. H., \& Siegel, J. M. (1978). Assessing the impact of life changes: development of the Life Experiences Survey. J Consult Clin Psychol, 46(5), 932.

Sassaroli, S., Lauro, L. J. R., Ruggiero, G. M., Mauri, M. C., Vinai, P., \& Frost, R. (2008). Perfectionism in depression, obsessive-compulsive disorder and eating disorders. Behaviour research and therapy, 46(6), 757-765.

Schuler, P. A. (2000). Perfectionism and gifted adolescents. Journal of secondary gifted education, 11(4), 183-196.

Shafran, R., \& Mansell, W. (2001). Perfectionism and psychopathology: A review of research and treatment. Clin Psychol Rev, 21(6), 879-906.

Sherry, S. B., Hewitt, P. L., Besser, A., McGee, B. J., \& Flett, (2004). Self-oriented and socially prescribed perfectionism in the Eating Disorder Inventory Perfectionism subscale. International Journal of Eating Disorders, 35(1), 69-79.

Sheykhi, Fathabadi, \& Heidari. (2013). The relations of anxiety, self-efficacy and perfectionism to dissertation procrastination. Developmental Psychology (Journal of Iranian Psychologists), 9(35), 283-295. 
Shrout, P. E., Link, B. G., Dohrenwend, B. P., Skodol, A. E., Stueve, A., \& Mirotznik, J. (1989). Characterizing life events as risk factors for depression: The role of fateful loss events. Journal of Abnormal Psychology, 98(4), 460.

Sironic, A., \& Reeve, R. A. (2012). More evidence for four perfectionism subgroups. Personality and Individual Differences, 53(4), 437-442.

Silverman, L. K. (1995, August). Perfectionism. Paper presented at the 11th World Conference on Gifted and Talented Children, Hong Kong.

Silverman, L. K. (2007). Perfectionism: The crucible of giftedness. Gifted Education International, 23(3), 233-245

Slade, P. D., \& Owens, R. G. (1998). A dual process model of perfectionism based on reinforcement theory. Behavior modification, 22(3), 372-390.

Slaney, R. B., Ashby, J. S., \& Trippi, J. (1995). Perfectionism: Its measurement and career relevance. Journal of career assessment, 3(4), 279-297.

Slaney, R. B., Rice, K. G., Mobley, M., Trippi, J., \& Ashby, J. S. (2001). The revised almost perfect scale. Measurement and evaluation in counseling and development, 34(3), 130.

Smith, C. A. (2018). "Re: Inquiry about 1993 Measure of Motivational Relevance, Motivational Congruence, and Accountability.” Message to Ansley Bender. 22 August 2018. E-mail.

Smith, \& Ellsworth, P. C. (1985). Patterns of cognitive appraisal in emotion. Journal of personality and social psychology, 48(4), 813.

Smith, C. A., Haynes, K. N., Lazarus, R. S., \& Pope, L. K. (1993). In search of the" hot" cognitions: Attributions, appraisals, and their relation to emotion. Journal of personality and social psychology, 65(5), 916. 
Smith, \& Lazarus, R. S. (1990). Emotion and adaptation. In L. A. Pervin (Eds.), Handbook of personality: Theory and research (609-637). New York: Guilford.

Smith, C. A., \& Lazarus, R. S. (1993). Appraisal components, core relational themes, and the emotions. Cognition \& Emotion, 7(3-4), 233-269.

Sherry, S. B., MacKinnon, A. L., Fossum, K. L., Antony, M. M., Stewart, S. H., Sherry, D. L., ... \& Mushquash, A. R. (2013). Perfectionism, discrepancies, and depression: Testing the perfectionism social disconnection model in a short-term, four-wave longitudinal study. Personality and Individual Differences, 54(6), 692-697.

Smith, M. M., Sherry, S. B., Chen, S., Saklofske, D. H., Mushquash, C., Flett, \& Hewitt, (2018). The Perniciousness of Perfectionism: A Meta-Analytic Review of the PerfectionismSuicide Relationship. Journal of Personality, 86(3), 522-542.

Smith, M. M., Sherry, S. B., McLarnon, M. E., Flett, G. L., Hewitt, P. L., Saklofske, D. H., \& Etherson, M. E. (2018). Why does socially prescribed perfectionism place people at risk for depression? A five-month, two-wave longitudinal study of the Perfectionism Social Disconnection Model. Personality and Individual Differences, 134, 49-54.

Smith, M. M., Sherry, S. B., Rnic, K., Saklofske, D. H., Enns, M., \& Gralnick, T. (2016). Are perfectionism dimensions vulnerability factors for depressive symptoms after controlling for neuroticism? A meta-analysis of 10 longitudinal studies. European Journal of Personality, 30(2), 201-212.

Smith, M. M., Sherry, S. B., Saklofske, D. H., \& Mushqaush, A. R. (2017). Clarifying the perfectionism-procrastination relationship using a 7-day, 14-occasion daily diary study. Personality and Individual Differences, 112, 117-123. 
Smith, M. M., Speth, T. A., Sherry, S. B., Saklofske, D. H., Stewart, S. H., \& Glowacka, M. (2017). Is socially prescribed perfectionism veridical? A new take on the stressfulness of perfectionism. Personality and Individual Differences, 110, 115-118.

Spector, P. E. (2006). Method variance in organizational research: Truth or urban legend? Organizational Research Methods, 9, 221-232.

Steel, P. (2007). The nature of procrastination: A meta-analytic and theoretical review of quintessential self-regulatory failure. Psychological bulletin, 133(1), 65.

Steiger, J. H. (1980). Tests for comparing elements of a correlation matrix. Psychological bulletin, 87(2), 245.

Stoeber, J., Feast, A. R., \& Hayward, J. A. (2009). Self-oriented and socially prescribed perfectionism: Differential relationships with intrinsic and extrinsic motivation and test anxiety. Personality and Individual Differences, 47(5), 423-428.

Streiner, D. L. (2003). Starting at the beginning: an introduction to coefficient alpha and internal consistency. Journal of personality assessment, 80(1), 99-103.

Sudler, E. L. (2014). Academic procrastination as mediated by executive functioning, perfectionism, and frustration intolerance in college students (Doctoral dissertation). Retreived from ProQuest Dissertations Publishing. (3575249).

Sun, J., Gan, Y., Liu, J., \& Chen, H. (2006). Mediation effect of self-efficacy on the relationship between perfectionism and coping strategies among graduate students. Chinese Journal of Clinical Psychology, 6.

Swartz, J. R., Knodt, A. R., Radtke, S. R., \& Hariri, A. R. (2015). A neural biomarker of psychological vulnerability to future life stress. Neuron, 85(3), 505-511. 
Tang, J., Wu, S., \& Miao, D. (2013). Experimental test of escape theory: Accessibility to implicit suicidal mind. Suicide and Life-Threatening Behavior, 43(4), 347-355.

Tang, P.-L., Wang, H.-H., \& Chou, F.-H. (2015). A systematic review and meta-analysis of demoralization and depression in patients with cancer. Psychosomatics, 56(6), 634-643.

Tavakol, M., \& Dennick, R. (2011). Making sense of Cronbach's alpha. International journal of medical education, 2, 53.

Taylor, P. J., Gooding, P., Wood, A. M., \& Tarrier, N. (2011). The role of defeat and entrapment in depression, anxiety, and suicide. Psychological bulletin, 137(3), 391.

Taylor, P. J., Wood, A. M., Gooding, P., \& Tarrier, N. (2010). Appraisals and suicidality: The mediating role of defeat and entrapment. Archives of Suicide Research, 14(3), 236-247.

Tecuta, L., Tomba, E., Grandi, S., \& Fava, G. A. (2015). Demoralization: a systematic review on its clinical characterization. Psychological medicine, 45(4), 673-691.

Tein, J.-Y., Sandler, I. N., \& Zautra, A. J. (2000). Stressful life events, psychological distress, coping, and parenting of divorced mothers: A longitudinal study. Journal of Family Psychology, 14(1), 27.

Tennant, C., \& Andrews, G. (1976). A scale to measure the stress of life events. Australian and New Zealand Journal of Psychiatry, 10(1), 27-32.

Tissot, A. M., \& Crowther, J. H. (2008). Self-oriented and socially prescribed perfectionism: Risk factors within an integrative model for bulimic symptomatology. Journal of Social and Clinical Psychology, 27(7), 734-755.

Törnblom, A. W., Werbart, A., \& Rydelius, P.-A. (2013). Shame behind the masks: The parents' perspective on their sons' suicide. Archives of Suicide Research, 17(3), 242-261. 
Tugade, M. M., \& Fredrickson, B. L. (2004). Resilient individuals use positive emotions to bounce back from negative emotional experiences. Journal of personality and social psychology, 86(2), 320 .

Utsey, S. O., \& Ponterotto, J. G. (1996). Development and validation of the Index of RaceRelated Stress (IRRS). Journal of Counseling Psychology, 43(4), 490.

Vaughn, C. E., \& Leff, J. P. (1981). Patterns of emotional response in relatives of schizophrenic patients. Schizophrenia Bulletin, 7(1), 43.

Vehling, S., Kissane, D. W., Lo, C., Glaesmer, H., Hartung, T. J., Rodin, G., \& Mehnert, A. (2017). The association of demoralization with mental disorders and suicidal ideation in patients with cancer. Cancer, 123(17), 3394-3401.

Vehling, S., Lehmann, C., Oechsle, K., Bokemeyer, C., Krüll, A., Koch, U., \& Mehnert, A. (2011). Global meaning and meaning-related life attitudes: exploring their role in predicting depression, anxiety, and demoralization in cancer patients. Supportive Care in Cancer, 19(4), 513-520.

Vehling, S., Lehmann, C., Oechsle, K., Bokemeyer, C., Krüll, A., Koch, U., \& Mehnert, A. (2012). Is advanced cancer associated with demoralization and lower global meaning? The role of tumor stage and physical problems in explaining existential distress in cancer patients. Psycho-Oncology, 21(1), 54-63.

Vehling, S., Oechsle, K., Koch, U., \& Mehnert, A. (2013). Receiving palliative treatment moderates the effect of age and gender on demoralization in patients with cancer. PLoS One, 8(3), e59417. 
Wang, K. T., Wong, Y. J., \& Fu, C. C. (2013). Moderation effects of perfectionism and discrimination on interpersonal factors and suicide ideation. Journal of Counseling Psychology, 60(3), 367.

Wetherall, K., Robb, K. A., \& O'Connor, (2018). An Examination of Social Comparison and Suicide Ideation Through the Lens of the Integrated Motivational-Volitional Model of Suicidal Behavior. Suicide and Life-Threatening Behavior.

Williams, J. M. G. (2001). Suicide and Attempted Suicide: Understanding the Cry of Pain. London: Penguin.

Williams, J. M. G., \& Pollock, L. R. (2008). The pschology of suicidal behaviour. In K. Hawton \& K. van Heeringen (Eds.), The international handbook of suicide and attempted suicide (79-93). New York, NY: Wiley \& Sons.

Wright, K. B. (2005). Researching Internet-based populations: Advantages and disadvantages of online survey research, online questionnaire authoring software packages, and web survey services. Journal of computer-mediated communication, 10(3), JCMC1034.

Wyatt, R., \& Gilbert, P. (1998). Dimensions of perfectionism: A study exploring their relationship with perceived social rank and status. Personality and Individual Differences, 24(1), 71-79.

Wyler, A. R., Masuda, M., \& Holmes, T. H. (1971). Magnitude of life events and seriousness of illness. Psychosomatic Medicine, 33(2), 115-122.

Yen, S., Pagano, M. E., Shea, M. T., Grilo, C. M., Gunderson, J. G., Skodol, A. E., . . Zanarini, M. C. (2005). Recent life events preceding suicide attempts in a personality disorder sample: findings from the collaborative longitudinal personality disorders study. $J$ Consult Clin Psychol, 73(1), 99. 
Zanicotti, C. G., Perez, D., \& Glue, P. (2012). Mood and pain responses to repeat dose intramuscular ketamine in a depressed patient with advanced cancer. Journal of palliative medicine, 15(4), 400-403. 


\section{Appendix A:}

\section{IRB Letter}

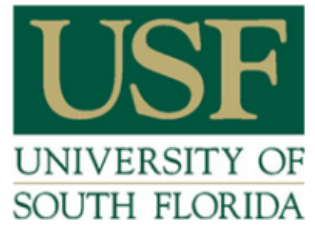

RESEARCH INTEGRITY AND COMPLIANCE

Institutional Review Boards, FWA No. 00001669

12901 Bruce B. Downs Blvd., MDC035 • Tampa, FL 33612-4799

(813) 974.5638 - FAX (813) 974.7091

\section{$1 / 11 / 2019$}

Ansley Bender

Psychology

14604 Gilligan's Way \#7

Tampa, FL 33613

\section{RE: Exempt Certification}

IRB\#: Pro00038577

Title: Perfectionism, Negative Life Events, and Cognitive Appraisal: A Contextual Model of Perfectionism's Maladaptive Nature

Dear Ms. Bender:

On 1/10/2019, the Institutional Review Board (IRB) determined that your research meets criteria for exemption from the federal regulations as outlined by 45CFR46.101(b):

(2) Research involving the use of educational tests (cognitive, diagnostic, aptitude, achievement), survey procedures, interview procedures or observation of public behavior, unless:

(i) information obtained is recorded in such a manner that human subjects can be identified, directly or through identifiers linked to the subjects; and (ii) any disclosure of the human subjects' responses outside the research could reasonably place the subjects at risk of criminal or civil liability or be damaging to the subjects' financial standing, employability, or reputation.

As the principal investigator for this study, it is your responsibility to ensure that this research is conducted as outlined in your application and consistent with the ethical principles outlined in the Belmont Report and with USF HRPP policies and procedures. 
Please note, as per USF HRPP Policy, once the Exempt determination is made, the application is closed in ARC. Any proposed or anticipated changes to the study design that was previously declared exempt from IRB review must be submitted to the IRB as a new study prior to initiation of the change. However, administrative changes, including changes in research personnel, do not warrant an amendment or new application.

Given the determination of exemption, this application is being closed in ARC. This does not limit your ability to conduct your research project.

We appreciate your dedication to the ethical conduct of human subject research at the University of South Florida and your continued commitment to human research protections. If you have

any questions regarding this matter, please call 813-974-5638.

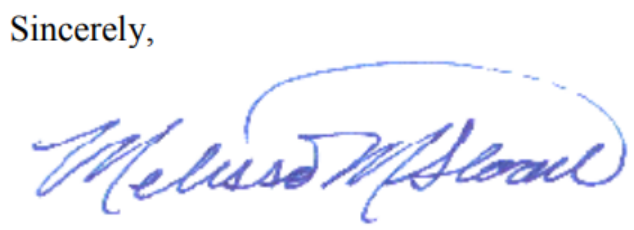

Melissa Sloan, $\mathrm{PhD}$, Vice Chairperson

USF Institutional Review Board 